\title{
ON COMPLETIONS OF HECKE ALGEBRAS
}

\author{
Maarten Solleveld \\ IMAPP, Radboud Universiteit \\ Heyendaalseweg 135, 6525AJ Nijmegen, the Netherlands \\ email: m.solleveld@science.ru.nl
}

\begin{abstract}
Let $G$ be a reductive $p$-adic group and let $\mathcal{H}(G)^{\mathfrak{s}}$ be a Bernstein block of the Hecke algebra of $G$. We consider two important topological completions of $\mathcal{H}(G)^{\mathfrak{s}}$ : a direct summand $\mathcal{S}(G)^{\mathfrak{s}}$ of the Harish-Chandra-Schwartz algebra of $G$ and a two-sided ideal $C_{r}^{*}(G)^{\mathfrak{s}}$ of the reduced $C^{*}$-algebra of $G$. These are useful for the study of all tempered smooth $G$-representations.

We suppose that $\mathcal{H}(G)^{\mathfrak{s}}$ is Morita equivalent to an affine Hecke algebra $\mathcal{H}(\mathcal{R}, q)$ - as is known in many cases. The latter algebra also has a Schwartz completion $\mathcal{S}(\mathcal{R}, q)$ and a $C^{*}$-completion $C_{r}^{*}(\mathcal{R}, q)$, both defined in terms of the underlying root datum $\mathcal{R}$ and the parameters $q$.

We prove that, under some mild conditions, a Morita equivalence $\mathcal{H}(G)^{\mathfrak{s}} \sim_{M}$ $\mathcal{H}(\mathcal{R}, q)$ extends to Morita equivalences $\mathcal{S}(G)^{\mathfrak{s}} \sim_{M} \mathcal{S}(\mathcal{R}, q)$ and $C_{r}^{*}(G)^{\mathfrak{s}} \sim_{M}$ $C_{r}^{*}(\mathcal{R}, q)$. We also check that our conditions are fulfilled in all known cases of such Morita equivalences between Hecke algebras. This is applied to compute the topological K-theory of the reduced $C^{*}$-algebra of a classical $p$-adic group.
\end{abstract}

\section{Contents}

Introduction

1. Affine Hecke algebras

1.1. The Schwartz and $C^{*}$-completions

1.2. The space of irreducible representations

2. Reductive $p$-adic groups

2.1. The Plancherel isomorphism

2.2. The space of irreducible representations 25

3. Morita equivalences

3.1. Conditions and first consequences 27

3.2. Preservation of temperedness 32

3.3. Comparison of completions 37

4. Hecke algebras from Bushnell-Kutzko types 40

5. Hecke algebras from Bernstein's progenerators 44

References 46

Date: August 31, 2018.

2010 Mathematics Subject Classification. 20C08, 22E50, 22E35.

The author is supported by a NWO Vidi grant "A Hecke algebra approach to the local Langlands correspondence" (nr. 639.032.528). 


\section{INTRODUCTION}

Let $G$ be a connected reductive group over a non-archimedean local field. Let $\operatorname{Rep}(G)$ be the category of smooth $G$-representations on complex vector spaces. To study such representations, it is often useful to consider various group algebras of $G$. Most fundamentally, there is the Hecke algebra $\mathcal{H}(G)$, the convolution algebra of locally constant, compactly supported functions $G \rightarrow \mathbb{C}$. The category $\operatorname{Rep}(G)$ is equivalent to the category $\operatorname{Mod}(\mathcal{H}(G))$ of nondegenerate $\mathcal{H}(G)$-modules. (Here $V$ nondegenerate means that $\mathcal{H}(G) \cdot V=V$.)

For purposes of harmonic analysis, and in particular for the study of tempered smooth $G$-representations, the Harish-Chandra-Schwartz algebra $\mathcal{S}(G)$ [HC] can be very convenient. This is a topological completion of $\mathcal{H}(G)$, it consists of locally constant functions $G \rightarrow \mathbb{C}$ that decay rapidly in a specified sense. By Wal, $\S I I I .7]$ an admissible smooth $G$-representation is tempered if and only if it is naturally a $\mathcal{S}(G)$-module. For larger representations it is best to define the category of tempered smooth $G$-representations as the category $\operatorname{Mod}(\mathcal{S}(G))$ of nondegenerate $\mathcal{S}(G)$-modules [SSZ, Appendix].

Further, from the point of view of operator algebras or noncommutative geometry, the reduced $C^{*}$-algebra $C_{r}^{*}(G)$ may be the most suitable. The modules of $C_{r}^{*}(G)$ are Banach spaces, so they are usually not smooth as $G$-representations. But $\mathcal{S}(G) \subset C_{r}^{*}(G)$ and the smooth vectors in any $C_{r}^{*}(G)$-module do form a nondegenerate $\mathcal{S}(G)$-module and hence a smooth $G$-representation. Moreover this operation provides a bijection between the irreducible representations of $C_{r}^{*}(G)$ and those of $\mathcal{S}(G)$. This feature distinguishes $C_{r}^{*}(G)$ from other Banach group algebras like $L^{1}(G)$ or the maximal $C^{*}$-algebra of $G$.

Let $\operatorname{Rep}(G)^{\mathfrak{s}}$ be a Bernstein block of $\operatorname{Rep}(G)$ [BeDe. It is well-known that in many cases (see Sections 4 and 5 ) $\operatorname{Rep}(G)^{\mathfrak{s}}$ is equivalent to the category of modules of an affine Hecke algebra $\mathcal{H}(\mathcal{R}, q)$. Here $\mathcal{R}$ is a root datum and $q$ is a parameter function for $\mathcal{R}$. In such cases it would be useful if one could detect, in terms of $\mathcal{H}(\mathcal{R}, q)$, whether a $G$-representation in $\operatorname{Rep}(G)^{\mathfrak{s}}$

(i) is tempered;

(ii) is unitary;

(iii) admits a continuous extension to a $C_{r}^{*}(G)$-module.

The structure needed to make sense of this is available for (extended) affine Hecke algebras with positive parameters. They have a natural *-operation, so unitarity is defined. Temperedness of finite-dimensional $\mathcal{H}(\mathcal{R}, q)$-modules can be defined conveniently either in terms of growth of matrix coefficient or by means of weights for a large commutative subalgebra of $\mathcal{H}(\mathcal{R}, q)$ [pd, §2.7].

Furthermore there exists a Schwartz completion $\mathcal{S}(\mathcal{R}, q)$ of $\mathcal{H}(\mathcal{R}, q)$ Opd, §6.2] with a similar structure as $\mathcal{S}(G)$ DeOp. By $[\mathrm{Opd}$, Corollary 6.7] a finite dimensional $\mathcal{H}(\mathcal{R}, q)$-module is tempered if and only if it extends continuously to a $\mathcal{S}(\mathcal{R}, q)$ module. Like for $G$, we define the category of tempered $\mathcal{H}(\mathcal{R}, q)$-modules to be the module category of $\mathcal{S}(\mathcal{R}, q)$.

The algebra $\mathcal{H}(\mathcal{R}, q)$ is a Hilbert algebra, so it acts by multiplication on its own Hilbert space completion. Then one can define the reduced $C^{*}$-completion $C_{r}^{*}(\mathcal{R}, q)$ as the closure of $\mathcal{H}(\mathcal{R}, q)$ in the algebra of bounded linear operators on that Hilbert space. It is reasonable to expect that this algebra plays a role analogous to $C_{r}^{*}(G)$. 
Let $\mathcal{H}(G)^{\mathfrak{s}}$ (resp. $\mathcal{S}(G)^{\mathfrak{s}}$ and $\left.C_{r}^{*}(G)^{\mathfrak{s}}\right)$ be the direct summand of $\mathcal{H}(G)$ (resp. $\mathcal{S}(G)$ and $\left.C_{r}^{*}(G)\right)$ corresponding to $\operatorname{Rep}(G)^{\mathfrak{s}}$ via the Bernstein decomposition

$$
\operatorname{Mod}(\mathcal{H}(G)) \cong \operatorname{Rep}(G)=\prod_{\mathfrak{s} \in \mathfrak{B}(G)} \operatorname{Rep}(G)^{\mathfrak{s}} .
$$

In view of the above, it is natural to ask whether an equivalence of categories

$$
\operatorname{Rep}(G)^{\mathfrak{s}} \cong \operatorname{Mod}\left(\mathcal{H}(G)^{\mathfrak{s}}\right) \stackrel{\sim}{\longrightarrow} \operatorname{Mod}(\mathcal{H}(\mathcal{R}, q))
$$

extends to Morita equivalences

$$
\mathcal{S}(G)^{\mathfrak{s}} \sim_{M} \mathcal{S}(\mathcal{R}, q) \quad \text { and } \quad C_{r}^{*}(G)^{\mathfrak{s}} \sim_{M} C_{r}^{*}(\mathcal{R}, q) .
$$

That would solve the issues (i) and (iii) completely, and would provide a partial answer to (ii). Namely, since irreducible tempered representations are unitary, it would imply that (1) matches the unitary tempered representations on both sides. (It is not clear what it could say about unitary non-tempered representations.) Furthermore (2) would make $\operatorname{Mod}\left(\mathcal{S}(G)^{\mathfrak{s}}\right)$ and $\operatorname{Mod}\left(C_{r}^{*}(G)^{\mathfrak{s}}\right)$ amenable to much more explicit calculations, in terms of the generators and relations from $\mathcal{H}(\mathcal{R}, q)$. That is important for topological K-theory, where one deals with finitely generated projective $C_{r}^{*}(G)$-modules.

While (2) looks fairly plausible, it is not automatic. To prove it, we impose some conditions on the Morita equivalence $\mathcal{H}(G)^{\mathfrak{s}} \sim_{M} \mathcal{H}(\mathcal{R}, q)$ :

- Condition 3.1 is about compatibility with parabolic induction and restriction.

- Condition 3.2 says roughly that under this Morita equivalence every (suitable) parabolic subgroup of $G$ should give rise to a parabolic subalgebra of $\mathcal{H}(\mathcal{R}, q)$, and this correspondence should respect positivity in the underlying root systems.

- Sometimes we obtain, instead of $\mathcal{H}(\mathcal{R}, q)$, an extended affine Hecke algebra $\mathcal{H}(\mathcal{R}, q) \rtimes \Gamma$, where $\Gamma$ is a finite group. Then we require Condition 1.1, which says that $\Gamma$ respects all the relevant structure.

Here Condition 3.1 has little to do with affine Hecke algebras. If it holds, then on based general principles. Condition 3.2 is needed to get affine Hecke algebras into play. If it does not hold, than our results simply cannot be formulated in such terms. The positivity part is innocent, usually it can be achieved by a good choice of a standard minimal parabolic subgroup of $G$. The Condition 1.1 is of a more technical nature, it serves to rule out some phenomena that could happen for arbitrary $\Gamma$ but not for reductive groups.

Notice that the above conditions do not say anything about *-homomorphisms between $\mathcal{H}(\mathcal{R}, q)$ and $\mathcal{H}(G)^{\mathfrak{s}}$. Instead, our condition are chosen so that they will hold true for affine Hecke algebras arising from reductive $p$-adic groups via the two main methods: Bushnell-Kutzko types and Bernstein's progenerators.

Theorem 1. (see Theorem 3.12)

Suppose that there is a Morita equivalence between $\mathcal{H}(G)^{\mathfrak{s}}$ and an extended affine Hecke algebra $\mathcal{H}(\mathcal{R}, q) \rtimes \Gamma$ with positive parameters, such that Conditions 3.1, 3.2 and 1.1 hold. Then it induces Morita equivalences $\mathcal{S}(G)^{\mathfrak{s}} \sim_{M} \mathcal{S}(\mathcal{R}, q)$ and $C_{r}^{*}(G)^{\mathfrak{s}} \sim_{M}$ $C_{r}^{*}(G)$.

Hitherto this was only proven for the Schwartz completions in the case of Iwahorispherical representations of split groups [DeOp, Theorem 10.2]. In all cases where a Morita equivalence on the Hecke algebra level is known (to the author), we check 
that the conditions of Theorem 1 are fulfilled. This includes principal series representations of $F$-split groups ( $F$ is any non-archimedean local field), level zero representations, inner forms of $\mathrm{GL}_{n}(F)$, inner forms of $\mathrm{SL}_{n}(F)$, symplectic groups (not necessarily split) and special orthogonal groups (also possibly non-split).

In all these cases we obtain a pretty good picture of $C_{r}^{*}(G)^{\mathfrak{s}}$, up to Morita equivalence. This can, for instance, be used to compute the topological K-theory of these algebras. Indeed, in Sol5] the author determined the K-theory of $C_{r}^{*}(\mathcal{R}, q)$ for many root data $\mathcal{R}$ (it does not depend on $q$ ). These calculations, together with Theorem 1 for classical groups, lead to a result which is useful in relation with the Baum-Connes conjecture.

Theorem 2. (see Theorem 5.3)

Let $G$ be a special orthogonal or a symplectic group over $F$ (not necessarily split), or an inner form of $\mathrm{GL}_{n}(F)$. Then $K_{*}\left(C_{r}^{*}(G)\right)$ is a free abelian group. For every Bernstein block $\operatorname{Rep}(G)^{\mathfrak{s}}$, the rank of $K_{*}\left(C_{r}(G)^{\mathfrak{s}}\right)$ is finite and can be computed explicitly.

Let us also discuss other approaches to the topics (i), (ii) and (iii) from page 2. In most cases where a Morita equivalence $\mathcal{H}(G)^{\mathfrak{s}} \sim_{M} \mathcal{H}(\mathcal{R}, q) \rtimes \Gamma$ is known, these issues are not discussed in the literature. When the Morita equivalence comes from a type $(K, \lambda)$ in the sense of Bushnell-Kutzko [BuKu, several relevant techniques are available. Let $e_{\lambda} \in \mathcal{H}(G)$ and $\mathcal{H}(G, J, \lambda)=\operatorname{End}_{G}\left(\operatorname{ind}_{J}^{G} \lambda\right)$ be the idempotent and the algebra associated to the type $(K, \lambda)$ in [BuKu, $\S 2]$. In this setting the Morita equivalence can be implemented by injective algebra homomorphisms

$$
\mathcal{H}(\mathcal{R}, q) \rtimes \Gamma \stackrel{\Upsilon_{\lambda}}{\longrightarrow} \mathcal{H}(G, J, \lambda) \rightarrow e_{\lambda} \mathcal{H}(G) e_{\lambda} \rightarrow \mathcal{H}(G)^{\mathfrak{s}},
$$

where we assume that $\Upsilon_{\lambda}$ is an isomorphism. It follows quickly from the definition of types that the last two maps in (3) induce Morita equivalences $\mathrm{BuKu},(2.12)$ and Theorem 4.3]. Both algebras $\mathcal{H}(G, J, \lambda)$ and $e_{\lambda} \mathcal{H}(G) e_{\lambda}$ are endowed with a natural trace and ${ }^{*}$, which are preserved by the injections

$$
\mathcal{H}(G, J, \lambda) \rightarrow e_{\lambda} \mathcal{H}(G) e_{\lambda} \rightarrow \mathcal{H}(G)^{\mathfrak{s}} .
$$

Considerations with Hilbert algebras show that the maps (4) induce equivalences between the associated categories of finite length tempered representations [BHK]. Moreover, after the first version of this paper appeared, Ciubotaru showed that (4) also induces equivalences between the respective subcategories of unitary modules Ciu. Hence, whenever $\Upsilon_{\lambda}$ is a ${ }^{*}$-isomorphism, the categories of unitary modules of all the algebras in (3) are equivalent. Earlier, this had been proven under certain additional conditions $\mathrm{BaMo}, \mathrm{BaCi}$.

Notice that in (3) all algebras are endowed with extra structure, which on the left hand side comes from affine Hecke algebras and for the other terms from the embedding in $\mathcal{H}(G)$. In particular both $\mathcal{H}(G)$ and $\mathcal{H}(\mathcal{R}, q) \rtimes \Gamma$ are endowed with a canonical trace, which stems from evaluation of functions at the unit element of $G$. Usually (3) will transfer the trace on $\mathcal{H}(G)^{\mathfrak{s}}$ to a positive scalar multiple of the trace on $\mathcal{H}(\mathcal{R}, q) \rtimes \Gamma$. If that is the case and $\Upsilon_{\lambda}$ is a ${ }^{*}$-isomorphism, then by DeOp, Theorem 10.1] (3) induces an equivalence between the category of finite length tempered $G$-representations in $\operatorname{Rep}(G)^{\mathfrak{s}}$ and the category of finite dimensional tempered modules of $\mathcal{H}(\mathcal{R}, q) \rtimes \Gamma$. This relies on properties of the Plancherel measures of $G$ and of $\mathcal{H}(\mathcal{R}, q) \rtimes \Gamma$, and it uses that $\Upsilon_{\lambda}$ preserves these Plancherel measures, up to a scalar multiple. 
When the Morita equivalence $\mathcal{H}(G)^{\mathfrak{s}} \sim_{M} \mathcal{H}(\mathcal{R}, q) \rtimes \Gamma$ does not arise from a type, fewer techniques for (i), (ii) and (iii) were known. Heiermann established such Morita equivalences, for symplectic and special orthogonal groups and for inner forms of $\mathrm{GL}_{n}(F)$ [Hei1, by using Bernstein's progenerators of $\operatorname{Rep}(G)^{\mathfrak{s}}$. In Hei2 he showed that these equivalences preserve temperedness of finite length modules. Here it is unknown whether the ${ }^{*}$ and the trace are preserved by the Morita equivalence. Since maps like (3) are lacking, it is even unclear how such a statement could be formulated in this setting.

Summarizing, in the literature already several results about the behaviour of finite length modules under a Morita equivalence between a Bernstein block $\operatorname{Rep}(G)^{\mathfrak{s}}$ and the module category of an (extended) affine Hecke algebra can be found, but there is so far almost nothing about the Schwartz completions and the $C^{*}$-completions.

Let us briefly describe the contents of the paper. In the first section we recall the definitions of affine Hecke algebras and their topological completions. We formulate the Plancherel isomorphism for these completions, from [DeOp], and we establish suitable versions for affine Hecke algebras extended with finite groups. We also analyse the space of irreducible representations and the subspace of irreducible tempered representations, mainly relying on [Sol3]. This is formulated in terms of the Langlands classification and induction from discrete series representations of parabolic subalgebras.

After that we look at the aforementioned group algebras for a reductive $p$-adic group $G$. We recall the Plancherel isomorphism for the Schwartz algebra of $G$ [HC, Wal and for the reduced $C^{*}$-algebra of $G[\mathrm{Ply}$. Like for affine Hecke algebras, we analyse the space of irreducible smooth $G$-representation in terms of the Langlands classification and parabolic induction of square-integrable representations, following Sol1.

This forms the setup for the proof of our main result Theorem 1, which occupies Section [3. The crucial idea behind our argument is that in the Plancherel isomorphisms for $\mathcal{S}(G)^{\mathfrak{s}}$ and $\mathcal{S}(\mathcal{R}, q)$ very similar algebras appear. In both settings one encounters a bundle of matrix algebras over a compact torus, one takes $C^{\infty}$-sections of those, and then invariants with respect to a finite group acting via intertwining operators. We compare the resulting algebras on both sides, analysing the data used to describe the Plancherel isomorphisms. First we prove that a Morita equivalence between $\mathcal{H}(G)^{\mathfrak{s}}$ and $\mathcal{H}(\mathcal{R}, q)$, plus the mild extra conditions listed on page 3, imply that the two necessary sets of data, for $\mathcal{S}(G)^{\mathfrak{s}}$ and for $\mathcal{S}(\mathcal{R}, q)$, become equivalent after some manipulations. The most problematic part is to prove that the Morita equivalences preserve temperedness and match square-integrable $G$-representations with discrete series $\mathcal{H}(\mathcal{R}, q)$-modules. For that we use very specific information about the spaces of irreducible representations and their subspaces of tempered representations. When we have compared all the data needed for the Plancherel isomorphisms on both sides, we establish the desired Morita equivalences between topological algebras.

In Section 4 we check that the conditions from Section 3 are fulfilled in (most) known cases of Morita equivalences coming from types. In the final section 5 we do the same for Heiermann's Morita equivalences constructed with the use of projective generators, and we derive Theorem 2 . 


\section{Affine Hecke algebras}

Let $\mathfrak{a}$ be a finite dimensional real vector space and let $\mathfrak{a}^{*}$ be its dual. Let $Y \subset \mathfrak{a}$ be a lattice and $X=\operatorname{Hom}_{\mathbb{Z}}(Y, \mathbb{Z}) \subset \mathfrak{a}^{*}$ the dual lattice. Let

$$
\mathcal{R}=\left(X, R, Y, R^{\vee}, \Delta\right) .
$$

be a based root datum. Thus $R$ is a reduced root system in $X, R^{\vee} \subset Y$ is the dual root system, $\Delta$ is a basis of $R$ and the set of positive roots is denoted $R^{+}$. Furthermore a bijection $R \rightarrow R^{\vee}, \alpha \mapsto \alpha^{\vee}$ is given, such that $\left\langle\alpha, \alpha^{\vee}\right\rangle=2$ and such that the corresponding reflections $s_{\alpha}: X \rightarrow X$ (resp. $s_{\alpha}^{\vee}: Y \rightarrow Y$ ) stabilize $R$ (resp. $\left.R^{\vee}\right)$. We do not assume that $R$ spans $\mathfrak{a}^{*}$. The reflections $s_{\alpha}$ generate the Weyl group $W=W(R)$ of $R$, and $S_{\Delta}:=\left\{s_{\alpha} \mid \alpha \in \Delta\right\}$ is the collection of simple reflections.

We have the affine Weyl group $W^{\text {aff }}=\mathbb{Z} R \rtimes W$ and the extended (affine) Weyl group $W^{e}=X \rtimes W$. Both can be considered as groups of affine transformations of $\mathfrak{a}^{*}$. We denote the translation corresponding to $x \in X$ by $t_{x}$. As is well-known, $W^{\text {aff }}$ is a Coxeter group, and the basis $\Delta$ of $R$ gives rise to a set $S^{\text {aff }}$ of simple (affine) reflections. More explicitly, let $\Delta_{M}^{\vee}$ be the set of maximal elements of $R^{\vee}$, with respect to the dominance ordering coming from $\Delta$. Then

$$
S^{\text {aff }}=S_{\Delta} \cup\left\{t_{\alpha} s_{\alpha} \mid \alpha^{\vee} \in \Delta_{M}^{\vee}\right\} .
$$

The length function $\ell$ of the Coxeter system ( $W^{\text {aff }}, S^{\text {aff }}$ ) extends naturally to $W^{e}$. The elements of length zero form a subgroup $\Omega \subset W^{e}$ and $W^{e}=W^{\text {aff }} \rtimes \Omega$.

A complex parameter function for $\mathcal{R}$ is a map $q: S^{\text {aff }} \rightarrow \mathbb{C}^{\times}$such that $q(s)=q\left(s^{\prime}\right)$ if $s$ and $s^{\prime}$ are conjugate in $W^{e}$. This extends naturally to a map $q: W^{e} \rightarrow \mathbb{C}^{\times}$ which is 1 on $\Omega$ and satisfies

$$
q\left(w w^{\prime}\right)=q(w) q\left(w^{\prime}\right) \quad \text { if } \quad \ell\left(w w^{\prime}\right)=\ell(w)+\ell\left(w^{\prime}\right) .
$$

Equivalently (see [Lus1, §3.1]) one can define $q$ as a $W$-invariant function

$$
q: R \cup\left\{2 \alpha: \alpha^{\vee} \in 2 Y\right\} \rightarrow \mathbb{C}^{\times} .
$$

We speak of equal parameters if $q(s)=q\left(s^{\prime}\right) \forall s, s^{\prime} \in S^{\text {aff }}$ and of positive parameters if $q(s) \in \mathbb{R}_{>0} \forall s \in S^{\text {aff }}$. We fix a square root $q^{1 / 2}: S^{\text {aff }} \rightarrow \mathbb{C}^{\times}$.

The affine Hecke algebra $\mathcal{H}=\mathcal{H}(\mathcal{R}, q)$ is the unique associative complex algebra with basis $\left\{N_{w} \mid w \in W^{e}\right\}$ and multiplication rules

$$
\begin{array}{ll}
N_{w} N_{w^{\prime}}=N_{w w^{\prime}} & \text { if } \quad \ell\left(w w^{\prime}\right)=\ell(w)+\ell\left(w^{\prime}\right), \\
\left(N_{s}-q(s)^{1 / 2}\right)\left(N_{s}+q(s)^{-1 / 2}\right)=0 & \text { if } \quad s \in S^{\text {aff }} .
\end{array}
$$

In the literature one also finds this algebra defined in terms of the elements $q(s)^{1 / 2} N_{s}$, in which case the multiplication can be described without square roots. This explains why $q^{1 / 2}$ does not appear in the notation $\mathcal{H}(\mathcal{R}, q)$. For $q=1$ (7) just reflects the defining relations of $W^{e}$, so $\mathcal{H}(\mathcal{R}, 1)=\mathbb{C}\left[W^{e}\right]$.

The set of dominant elements in $X$ is

$$
X^{+}=\left\{x \in X:\left\langle x, \alpha^{\vee}\right\rangle \geq 0 \forall \alpha \in \Delta\right\} .
$$

The subset $\left\{N_{t_{x}}: x \in X^{+}\right\} \subset \mathcal{H}(\mathcal{R}, q)$ is closed under multiplication, and isomorphic to $X^{+}$as a semigroup. For any $x \in X$ we put

$$
\theta_{x}=N_{t_{x_{1}}} N_{t_{x_{2}}}^{-1}, \text { where } x_{1}, x_{2} \in X^{+} \text {and } x=x_{1}-x_{2} .
$$

This does not depend on the choice of $x_{1}$ and $x_{2}$, so $\theta_{x} \in \mathcal{H}(\mathcal{R}, q)^{\times}$is well-defined. The Bernstein presentation of $\mathcal{H}(\mathcal{R}, q)$ [Lus1, §3] says that: 
- $\left\{\theta_{x}: x \in X\right\}$ forms a $\mathbb{C}$-basis of a subalgebra of $\mathcal{H}(\mathcal{R}, q)$ isomorphic to $\mathbb{C}[X] \cong \mathcal{O}(T)$, which we identify with $\mathcal{O}(T)$.

- $\mathcal{H}(W, q):=\mathbb{C}\left\{N_{w}: w \in W\right\}$ is a finite dimensional subalgebra of $\mathcal{H}(\mathcal{R}, q)$ (known as the Iwahori-Hecke algebra of $W$ ).

- The multiplication map $\mathcal{O}(T) \otimes \mathcal{H}(W, q) \rightarrow \mathcal{H}(\mathcal{R}, q)$ is a $\mathbb{C}$-linear bijection.

- There are explicit cross relations between $\mathcal{H}(W, q)$ and $\mathcal{O}(T)$, deformations of the standard action of $W$ on $\mathcal{O}(T)$.

To define parabolic subalgebras of affine Hecke algebras, we associate some objects to any set of simple roots $Q \subset \Delta$. Let $R_{Q}$ be the root system they generate, $R_{Q}^{\vee}$ the root system generated by $Q^{\vee}$ and $W_{Q}$ their Weyl group. We also define

$$
\begin{array}{ll}
X_{Q}=X /\left(X \cap\left(Q^{\vee}\right)^{\perp}\right) & X^{Q}=X /(X \cap \mathbb{Q} Q), \\
Y_{Q}=Y \cap \mathbb{Q}^{\vee} & Y^{Q}=Y \cap Q^{\perp}, \\
T_{Q}=\operatorname{Hom}_{\mathbb{Z}}\left(X_{Q}, \mathbb{C}^{\times}\right) & T^{Q}=\operatorname{Hom}_{\mathbb{Z}}\left(X^{Q}, \mathbb{C}^{\times}\right), \\
\mathfrak{a}_{Q}=Y_{Q} \otimes_{\mathbb{Z}} \mathbb{R} & \mathfrak{a}^{Q}=Y^{Q} \otimes_{\mathbb{Z}} R, \\
\mathcal{R}_{Q}=\left(X_{Q}, R_{Q}, Y_{Q}, R_{Q}^{\vee}, Q\right) & \mathcal{R}^{Q}=\left(X, R_{Q}, Y, R_{Q}^{\vee}, Q\right), \\
\mathcal{H}_{Q}=\mathcal{H}\left(\mathcal{R}_{Q}, q_{Q}\right) & \mathcal{H}^{Q}=\mathcal{H}\left(\mathcal{R}^{Q}, q^{Q}\right) .
\end{array}
$$

Here $q_{Q}$ and $q^{Q}$ are derived from $q$ via (6). Both $\mathcal{H}_{Q}$ and $\mathcal{H}^{Q}$ are called parabolic subalgebras of $\mathcal{H}$. The quotient map $X \mapsto X_{Q}$ yields a natural projection

$$
\mathcal{H}^{Q} \rightarrow \mathcal{H}_{Q}: \theta_{x} N_{w} \mapsto \theta_{x_{Q}} N_{w} .
$$

In this way one can regard $\mathcal{H}_{Q}$ as a "semisimple" quotient of $\mathcal{H}^{Q}$. The algebra $\mathcal{H}^{Q}$ is embedded in $\mathcal{H}$ via the Bernstein presentation, as the image of $\mathcal{O}(T) \otimes \mathcal{H}\left(W_{Q}, q\right) \rightarrow$ $\mathcal{H}$. Any $t \in T^{Q}$ and any $u \in T^{Q} \cap T_{Q}$ give rise to algebra automorphisms

$$
\begin{aligned}
\psi_{u}: \mathcal{H}_{Q} \rightarrow \mathcal{H}_{Q}, \quad \theta_{x_{Q}} N_{w} & \mapsto u\left(x_{Q}\right) \theta_{x_{Q}} N_{w}, \\
\psi_{t}: \mathcal{H}^{Q} \rightarrow \mathcal{H}^{Q}, \quad \theta_{x} N_{w} & \mapsto t(x) \theta_{x} N_{w} .
\end{aligned}
$$

Let $\Gamma$ be a finite group acting on $\mathcal{R}$, i.e. it acts $\mathbb{Z}$-linearly on $X$ and preserves $R$ and $\Delta$. We also assume that $\Gamma$ acts on $T$ by affine transformations, whose linear part comes from the action on $X$. Thus $\Gamma$ acts on $\mathcal{O}(T) \cong \mathbb{C}[X]$ by

$$
\gamma\left(\theta_{x}\right)=z_{\gamma}(x) \theta_{\gamma x}
$$

for some $z_{\gamma} \in T$. We suppose throughout that $q^{1 / 2}$ is $\Gamma$-invariant, and that $\Gamma$ acts on $\mathcal{H}(\mathcal{R}, q)$ by the algebra automorphisms

$$
\operatorname{Ad}(\gamma): \sum_{w \in W, x \in X} c_{x, w} \theta_{x} N_{w} \mapsto \sum_{w \in W, x \in X} c_{x, w} z_{\gamma}(x) \theta_{\gamma(x)} N_{\gamma w \gamma^{-1}}
$$

This being a group action, the multiplication relations in $\mathcal{H}(\mathcal{R}, q)$ imply that we must have $z_{\gamma} \in T^{W}$. We build the crossed product algebra

$$
\mathcal{H}(\mathcal{R}, q) \rtimes \Gamma .
$$

In Sol3 we considered a slightly less general action of $\Gamma$ on $\mathcal{H}(\mathcal{R}, q)$, where the elements $z_{\gamma} \in T^{W}$ from (10) were all equal to 1 . But the relevant results from [Sol3] do not rely on $\Gamma$ fixing the unit element of $T$, so they are also valid for the actions as in (11). In this paper we will tacitly use some results from [Sol3] in the generality of (11). We note that nontrivial $z_{\gamma} \in T^{W}$ are sometimes needed to describe Hecke algebras coming from $p$-adic groups, for example in [Roc2, §4]. 
Since $\mathcal{H}(\mathcal{R}, q)$ is of finite rank as a module over its commutative subalgebra $\mathcal{O}(T)$, all irreducible $\mathcal{H}(\mathcal{R}, q)$-modules have finite dimension. The set of $\mathcal{O}(T)$-weights of a $\mathcal{H}(\mathcal{R}, q)$-module $V$ will be denoted by $\mathrm{Wt}(V)$.

We regard $\mathfrak{t}=\mathfrak{a} \oplus i \mathfrak{a}$ as the polar decomposition of $\mathfrak{t}$, with associated real part map $\Re: \mathfrak{t} \rightarrow \mathfrak{a}$. The vector space $\mathfrak{t}$ can be interpreted as the Lie algebra of the complex torus $T=\operatorname{Hom}_{\mathbb{Z}}\left(X, \mathbb{C}^{\times}\right)$. The latter has a polar decomposition $T=T_{\mathrm{rs}} \times T_{\text {un }}$ where $T_{\mathrm{rs}}=\operatorname{Hom}_{\mathbb{Z}}\left(X, \mathbb{R}_{>0}\right)$ and $T_{\mathrm{un}}=\operatorname{Hom}_{\mathbb{Z}}\left(X, S^{1}\right)$ is the unique maximal compact subgroup of $T$. The polar decomposition of an element $t \in T$ is written as $t=$ $|t|\left(t|t|^{-1}\right)$. The exponential map exp $: \mathfrak{t} \rightarrow T$ becomes bijective when restricted to $\mathfrak{a} \rightarrow T_{\mathrm{rs}}$. We denote its inverse by $\log : T_{\mathrm{rs}} \rightarrow \mathfrak{a}$.

We write

$$
\begin{aligned}
& \mathfrak{a}^{+}=\{\mu \in \mathfrak{a}:\langle\alpha, \mu\rangle \geq 0 \forall \alpha \in \Delta\}, \\
& \mathfrak{a}^{*+}=\left\{x \in \mathfrak{a}^{*}:\left\langle x, \alpha^{\vee}\right\rangle \geq 0 \forall \alpha \in \Delta\right\}, \\
& \mathfrak{a}^{-}=\left\{\lambda \in \mathfrak{a}:\langle x, \lambda\rangle \leq 0 \forall x \in \mathfrak{a}^{*+}\right\}=\left\{\sum_{\alpha \in \Delta} \lambda_{\alpha} \alpha^{\vee}: \lambda_{\alpha} \leq 0\right\} .
\end{aligned}
$$

The interior $\mathfrak{a}^{--}$of $\mathfrak{a}^{-}$equals $\left\{\sum_{\alpha \in \Delta} \lambda_{\alpha} \alpha^{\vee}: \lambda_{\alpha}<0\right\}$ if $\Delta$ spans $\mathfrak{a}^{*}$, and is empty otherwise. We write

$$
T^{-}=\exp \left(\mathfrak{a}^{-}\right) \subset T_{\mathrm{rs}} \quad \text { and } \quad T^{--}=\exp \left(\mathfrak{a}^{--}\right) \subset T_{\mathrm{rs}} .
$$

We say that a module $V$ for $\mathcal{H}(\mathcal{R}, q)$ (or for $\mathcal{H}(\mathcal{R}, q) \rtimes \Gamma$ ) is tempered if $|\mathrm{Wt}(V)| \subset$ $T^{-}$, and that it is discrete series if $|\mathrm{Wt}(V)| \subset T^{--}$. The latter is only possible if $R$ spans $\mathfrak{a}$, for otherwise $\mathfrak{a}^{--}$and $T^{--}$are empty. We alleviate these notions by calling a $\mathcal{H} \rtimes \Gamma$-module essentially discrete series if its restriction to $\mathcal{H}_{\Delta}$ is discrete series. Equivalently, essentially discrete series means that $\mathrm{Wt}(V) \subset T^{--} T_{\text {un }} T^{\Delta}$. Such a representation is tempered if and only if $\mathrm{Wt}(V) \subset T^{--} T_{\mathrm{un}}$. We denote the set of (equivalence classes of) irreducible tempered essentially discrete series representations by $\operatorname{Irr}_{L^{2}}(\mathcal{H}(\mathcal{R}, q) \rtimes \Gamma)$.

It follows from the Bernstein presentation [Lus1, §3] that

$$
\text { the centre of } \mathcal{H}(\mathcal{R}, q) \rtimes \Gamma \text { contains } \mathcal{O}(T)^{W \Gamma}=\mathcal{O}(T / W \Gamma),
$$

with equality if $W \Gamma$ acts faithfully on $T$. By Schur's Lemma $\mathcal{O}(T)^{W \Gamma}$ acts on every irreducible $\mathcal{H} \rtimes \Gamma$-representation $\pi$ by a character. Such a character can be identified with a $W \Gamma$-orbit $W \Gamma t \subset T$. We will just call $W \Gamma t$ the central character of $\pi$. Then $W \Gamma|t| \subset T_{\text {rs }}$ and $c c(\pi):=W \Gamma \log |t|$ is a single $W \Gamma$-orbit in $\mathfrak{a}$. We fix a $W \Gamma$-invariant inner product on $\mathfrak{a}$ and we define

$$
\|c c(\pi)\|=\|\log |t|\| .
$$

In some cases that we will encounter, the appropriate parabolic subalgebras of $\mathcal{H}(\mathcal{R}, q) \rtimes \Gamma$ are not $\mathcal{H}\left(\mathcal{R}^{Q}, q^{Q}\right)$, but $\mathcal{H}\left(\mathcal{R}^{Q}, q^{Q}\right) \rtimes \Gamma_{Q}$ for some subgroup $\Gamma_{Q} \subset \Gamma$. To make this work well, we need some assumptions on the groups $\Gamma_{Q}$ for $Q \subset \Delta$.

Condition 1.1. (1) $\Gamma_{Q} \subset \Gamma_{Q^{\prime}}$ if $Q \subset Q^{\prime}$;

(2) the action of $\Gamma_{Q}$ on $T$ stabilizes $T_{Q}$ and $T^{Q}$;

(3) $\Gamma_{Q}$ acts on $T^{Q}$ by multiplication with elements of $K_{Q}$.

Notice that $\Gamma_{Q}$ is a subgroup of $\Gamma(Q, Q)=\{\gamma \in \Gamma: \gamma(Q)=Q\}$, but that we do not require these two groups to be equal. 
We also note that Conditions 1.1 entail that $\Gamma_{\emptyset}$ acts trivially on $\mathcal{O}(T)=\mathcal{H}^{\emptyset}$, so

$$
\operatorname{Irr}\left(\mathcal{H}^{\emptyset} \rtimes \Gamma_{\emptyset}\right) \cong T \times \operatorname{Irr}\left(\Gamma_{\emptyset}\right) .
$$

Remark 1.2. Often there is a larger root system $\tilde{R} \supset R$ in $X$, such that $W_{Q} \Gamma_{Q}$ is contained in the parabolic subgroup of $W(\tilde{R})$ associated to $\tilde{R} \cap \mathbb{Q} Q$. Then parts (2) and (3) of Condition 1.1 are automatically satisfied (and part (1) is usually obvious).

Under Conditions $1.1 \Gamma_{Q}$ commutes with $K_{Q}$, The conditions also entail that the projection $\mathcal{H}^{Q} \rightarrow \mathcal{H}_{Q}$ and the isomorphisms $\phi_{t}: \mathcal{H}^{Q} \rightarrow \mathcal{H}^{Q}\left(t \in T^{Q}\right)$ are $\Gamma_{Q^{-}}$equivariant, so they extend to algebra homomorphisms

$$
\mathcal{H}^{Q} \rtimes \Gamma_{Q} \rightarrow \mathcal{H}_{Q} \rtimes \Gamma_{Q} \text { and } \phi_{t}: \mathcal{H}^{Q} \rtimes \Gamma_{Q} \rightarrow \mathcal{H}^{Q} \rtimes \Gamma_{Q}\left(t \in T^{Q}\right) .
$$

Via the first map of (16) we can inflate any representation of $\mathcal{H}_{Q} \rtimes \Gamma_{Q}$ to $\mathcal{H}^{Q} \rtimes \Gamma_{Q}$, which we often do tacitly. For any representation $\pi$ of $\mathcal{H}^{Q} \rtimes \Gamma_{Q}$ and any $t \in T^{Q}$ we write

$$
\pi \otimes t=\pi \circ \phi_{t} \in \operatorname{Mod}\left(\mathcal{H}^{Q} \rtimes \Gamma_{Q}\right) .
$$

Lemma 1.3. (a) Every irreducible $\mathcal{H}^{Q} \rtimes \Gamma_{Q}$-representation is of the form $\pi_{Q} \otimes t^{Q}$ for some $\pi_{Q} \in \operatorname{Irr}\left(\mathcal{H}_{Q} \rtimes \Gamma_{Q}\right)$ and $t^{Q} \in T^{Q}$.

(b) $\pi_{Q} \otimes t^{Q}$ is tempered if and only if $\pi$ is tempered and $t^{Q} \in T_{\mathrm{un}}^{Q}$.

(c) $\pi_{Q} \otimes t^{Q}$ is essentially discrete series if and only if $\pi_{Q}$ is discrete series.

Proof. (a) First we consider the situation without $\Gamma_{Q}$. Let $\pi \in \operatorname{Irr}\left(\mathcal{H}^{Q}\right)$ with central character $W_{Q} t \in T / W_{Q}$. The group $W_{Q}=W\left(R_{Q}\right)$ acts trivially on $T^{Q}$, so $W_{Q} t=$ $t^{Q} W_{Q} t_{Q}$ for some $t^{Q} \in T^{Q}, t_{Q} \in T_{Q}$. Then $\pi \otimes\left(t^{Q}\right)^{-1}$ factors through $\mathcal{H}^{Q} \rightarrow \mathcal{H}_{Q}$ (say as $\pi_{Q}$ ), and $\pi=\pi_{Q} \otimes t^{Q}$.

To include $\Gamma_{Q}$ we use Clifford theory [RaRa, Theorem A.6]. It says that every irreducible $\mathcal{H}^{Q} \rtimes \Gamma_{Q}$-representation is of the form

$$
\pi \rtimes \rho:=\operatorname{ind}_{\mathcal{H}^{Q} \rtimes \Gamma_{Q, \pi}}^{\mathcal{H}^{Q} \rtimes \Gamma_{Q}}(\pi \otimes \rho) .
$$

Here $\Gamma_{Q, \pi}$ is the stabilizer of $\pi \in \operatorname{Irr}\left(\mathcal{H}^{Q}\right)$ in $\Gamma_{Q}$ and $\left(\rho, V_{\rho}\right)$ is an irreducible representation of a twisted group algebra of $\Gamma_{Q, \pi}$. If $\mathcal{O}(T)$ acts by $t^{Q} t_{1}$ on a vector subspace $V_{1} \subset V_{\pi}$, then for $\gamma \in \Gamma_{Q}$ it acts by the character $\gamma^{-1}\left(t^{Q} t_{1}\right)$ on $N_{\gamma}\left(V_{1} \otimes V_{\rho}\right)$. By Condition 1.1. (3) $\gamma^{-1}\left(t^{Q} t_{1}\right) \in t^{Q} K_{Q} \gamma^{-1}\left(t_{1}\right)$. Hence $(\pi \rtimes \rho) \otimes\left(t^{Q}\right)^{-1}$ factors through $\mathcal{H}^{Q} \rtimes \Gamma_{Q} \rightarrow \mathcal{H}_{Q} \rtimes \Gamma_{Q}$ as $\pi_{Q} \rtimes \rho$, and

$$
\pi \rtimes \rho=\left(\pi_{Q} \rtimes \rho\right) \otimes t^{Q} .
$$

(b) As $T=T^{Q} T_{Q}$ with $T^{Q} \cap T_{Q} \subset T_{\text {un }}$, there is a factorization $T_{r s}=T_{r s}^{Q} \times T_{Q, r s}$ and (with respect to $\mathcal{R}^{Q}$ ) $T_{r s}^{-}=\{1\} \times T_{Q, r s}^{-}$. Also $\left|\mathrm{Wt}\left(\pi \otimes t^{Q}\right)\right|=\left|t^{Q}\right||\mathrm{Wt}(\pi)|$. These observations imply the result.

(c) This is obvious from $\mathrm{Wt}\left(\pi \otimes t^{Q}\right)=t^{Q} \mathrm{Wt}(\pi)$.

\subsection{The Schwartz and $C^{*}$-completions.}

To get nice completions of $\mathcal{H}(\mathcal{R}, q)$ we assume from now on that $q$ is a positive parameter function for $\mathcal{R}$. As a topological vector space the Schwartz completion of $\mathcal{H}(\mathcal{R}, q)$ will consist of rapidly decreasing functions on $W^{e}$, with respect to a suitable length function $\mathcal{N}$. For example we can take a $W$-invariant norm on $X \otimes_{\mathbb{Z}} \mathbb{R}$ and 
put $\mathcal{N}\left(w t_{x}\right)=\|x\|$ for $w \in W$ and $x \in X$. Then we can define, for $n \in \mathbb{N}$, the following norm on $\mathcal{H}$ :

$$
p_{n}\left(\sum_{w \in W^{e}} h_{w} N_{w}\right)=\sup _{w \in W^{e}}\left|h_{w}\right|(\mathcal{N}(w)+1)^{n} .
$$

The completion of $\mathcal{H}$ with respect to these norms is the Schwartz algebra $\mathcal{S}=$ $\mathcal{S}(\mathcal{R}, q)$. It is known from $\mathrm{Opd}$, Section 6.2] that it is a Fréchet algebra. The $\Gamma$ action on $\mathcal{H}$ extends continuously to $\mathcal{S}$, so the crossed product algebra $\mathcal{S}(\mathcal{R}, q) \rtimes \Gamma$ is well-defined. By $\mathrm{Opd}$, Lemma 2.20] a finite dimensional $\mathcal{H} \rtimes \Gamma$-representation is tempered if and only if it extends continuously to an $\mathcal{S} \rtimes \Gamma$-representation.

We define a ${ }^{*}$-operation and a trace on $\mathcal{H}(\mathcal{R}, q)$ by

$$
\begin{aligned}
\left(\sum_{w \in W^{e}} c_{w} N_{w}\right)^{*} & =\sum_{w \in W^{e}} \overline{c_{w}} N_{w^{-1}}, \\
\tau\left(\sum_{w \in W^{e}} c_{w} N_{w}\right) & =c_{e} .
\end{aligned}
$$

Since $q\left(s_{\alpha}\right)>0,{ }^{*}$ preserves the relations (7) and defines an anti-involution of $\mathcal{H}(\mathcal{R}, q)$. The set $\left\{N_{w}: w \in W^{e}\right\}$ is an orthonormal basis of $\mathcal{H}(\mathcal{R}, q)$ for the inner product

$$
\left\langle h_{1}, h_{2}\right\rangle=\tau\left(h_{1}^{*} h_{2}\right) .
$$

This gives $\mathcal{H}(\mathcal{R}, q)$ the structure of a Hilbert algebra. The Hilbert space completion $L^{2}(\mathcal{R})$ of $\mathcal{H}(\mathcal{R}, q)$ is a module over $\mathcal{H}(\mathcal{R}, q)$, via left multiplication. Moreover every $h \in \mathcal{H}(\mathcal{R}, q)$ acts as a bounded linear operator $\mathrm{Opd}$, Lemma 2.3]. The reduced $C^{*}$-algebra of $\mathcal{H}(\mathcal{R}, q)$ Opd, $\left.\S 2.4\right]$, denoted $C_{r}^{*}(\mathcal{R}, q)$, is defined as the closure of $\mathcal{H}(\mathcal{R}, q)$ in the algebra of bounded linear operators on $L^{2}(\mathcal{R})$. By Opd, Theorem $6.1]$

$$
\mathcal{H}(\mathcal{R}, q) \subset \mathcal{S}(\mathcal{R}, q) \subset C_{r}^{*}(\mathcal{R}, q) .
$$

As in (12), we can extend this to a $C^{*}$-algebra $C_{r}^{*}(\mathcal{R}, q) \rtimes \Gamma$, provided that $q$ is $\Gamma$-invariant.

Let us recall some background about $C_{r}^{*}(\mathcal{R}, q) \rtimes \Gamma$, mainly from $\mathrm{Opd}$, Sol3]. It follows from DeOp, Corollary 5.7] that it is a finite type I $C^{*}$-algebra and that $\operatorname{Irr}\left(C_{r}^{*}(\mathcal{R}, q)\right)$ is precisely the tempered part of $\operatorname{Irr}(\mathcal{H}(\mathcal{R}, q))$. According to $\mathrm{Opd}$, Theorem 4.23] all irreducible $\mathcal{S}(\mathcal{R}, q) \rtimes \Gamma$-representations extend continuously to $C_{r}^{*}(\mathcal{R}, q) \rtimes \Gamma$. Hence we can regard the representation theory of $C_{r}^{*}(\mathcal{R}, q) \rtimes \Gamma$ as the tempered unitary representation theory of $\mathcal{H}(\mathcal{R}, q) \rtimes \Gamma)$.

The structure of $C_{r}^{*}(\mathcal{R}, q) \rtimes \Gamma$ is described in terms of parabolically induced representations. As induction data we use triples $(Q, \delta, t)$ where $Q \subset \Delta, \delta \in \operatorname{Irr}_{L^{2}}\left(\mathcal{H}_{Q}\right)$ and $t \in T^{Q}$. We regard two triples $(Q, \delta, t)$ and $\left(Q^{\prime}, \delta^{\prime}, t^{\prime}\right)$ as equivalent if $Q=$ $Q^{\prime}, t=t^{\prime}$ and $\delta \cong \delta^{\prime}$. Notice that $\mathcal{H}_{Q}$ comes from a semisimple root datum, so it can have discrete series representations. We inflate such a representation to $\mathcal{H}^{Q}$ via the projection (8). To a triple $(Q, \delta, t)$ we associate the $\mathcal{H} \rtimes \Gamma$-representation

$$
\pi^{\Gamma}(Q, \delta, t)=\operatorname{ind}_{\mathcal{H}^{Q}}^{\mathcal{H} \times \Gamma}\left(\delta \circ \psi_{t}\right) .
$$

(When $\Gamma=1$, we often suppress it from these and similar notations.) For $t \in T_{\mathrm{un}}^{Q}=$ $T^{Q} \cap T_{\text {un }}$ these representations extend continuously to the respective $C^{*}$-completions of the involved algebras. Let $\Xi_{\text {un }}$ be the set of triples $(Q, \delta, t)$ as above, such that moreover $t \in T_{\text {un }}$. Considering $Q$ and $\delta$ as discrete variables, we regard $\Xi_{\text {un }}$ as a disjoint union of finitely many compact real tori (of different dimensions). 
Let $\mathcal{V}_{\Xi}^{\Gamma}$ be the vector bundle over $\Xi_{\text {un }}$, whose fibre at $\xi=(Q, \delta, t)$ is the vector space underlying $\pi^{\Gamma}(Q, \delta, t)$. That vector space is independent of $t$, so the vector bundle is trivial. Let $\operatorname{End}\left(\mathcal{V}_{\Xi}^{\Gamma}\right)$ be the algebra bundle with fibres $\operatorname{End}_{\mathbb{C}}\left(\pi^{\Gamma}(Q, \delta, t)\right)$. These data give rise to a canonical map

$$
\begin{array}{cl}
\mathcal{H}(\mathcal{R}, q) \rtimes \Gamma & \rightarrow \mathcal{O}(\Xi ; \operatorname{End}(\mathcal{V} \Gamma)) \\
h & \mapsto\left(\xi \mapsto \pi^{\Gamma}(\xi)(h)\right)
\end{array}
$$

which we refer to as the Fourier transform. By $\mathrm{Opd}$, Lemma 2.22] every discrete series representation is unitary, so $V_{\delta}$ carries an $\mathcal{H}_{Q}$-invariant inner product and $\operatorname{End}_{\mathbb{C}}\left(V_{\delta}\right)$ has a natural ${ }^{*}$-operation. For any $t \in T^{Q}$ this becomes an $\mathcal{H}^{Q}$-invariant nondegenerate pairing between $\delta \circ \phi_{t}$ and $\delta \circ \phi_{t|t|^{-2}}$. By $\mathrm{Opd}$, Proposition 4.19] this extends canonically to an inner product on the vector space

$$
\pi^{\Gamma}(Q, \delta, t)=\Gamma \ltimes \mathcal{H}(W, q) \otimes_{\mathcal{H}\left(W_{Q}, q\right)} V_{\delta} .
$$

That yields an anti-involution on $\operatorname{End}_{\mathbb{C}}\left(\pi^{\Gamma}(Q, \delta, t)\right)$ and a nondegenerate $\mathcal{H} \rtimes \Gamma$ invariant pairing between $\pi^{\Gamma}(Q, \delta, t)$ and $\pi^{\Gamma}\left(Q, \delta, t|t|^{-2}\right)$.

The algebra $\mathcal{O}\left(\Xi ; \operatorname{End}\left(\mathcal{V}_{\Xi}^{\Gamma}\right)\right)$ is endowed with the anti-involution

$$
\left(f^{*}\right)(Q, \delta, t)=f\left(Q, \delta, t|t|^{-2}\right)^{*} .
$$

With respect to this anti-involution, (18) is a ${ }^{*}$-homomorphism.

To administer the upcoming intertwining operators we use a finite groupoid $\mathcal{G}$ which acts on $\operatorname{End}\left(\mathcal{V}_{\Xi}^{\Gamma}\right)$. It is made from elements of $W \rtimes \Gamma$ and of $K_{Q}:=T_{Q} \cap T^{Q}$. More precisely, its base space is the power set of $\Delta$, and for $Q, Q^{\prime} \subseteq \Delta$ the collection of arrows from $Q$ to $Q^{\prime}$ is

$$
\mathcal{G}_{Q Q^{\prime}}=\left\{(g, u): g \in \Gamma \ltimes W, u \in K_{Q}, g(Q)=Q^{\prime}\right\} .
$$

Whenever it is defined, the multiplication in $\mathcal{G}$ is

$$
\left(g^{\prime}, u^{\prime}\right) \cdot(g, u)=\left(g^{\prime} g, g^{-1}\left(u^{\prime}\right) u\right) .
$$

In particular, writing $W \Gamma(Q, Q)=\{w \in W \Gamma: w(Q)=Q\}$, we have the group

$$
\mathcal{G}_{Q Q}=W \Gamma(Q, Q) \rtimes K_{Q} .
$$

Usually we will write elements of $\mathcal{G}$ simply as $g u$. There is an analogous groupoid $\mathcal{G}^{Q}$ for $\mathcal{H}^{Q} \rtimes \Gamma_{Q}$, which under Conditions 1.1 satisfies $\mathcal{G}_{Q Q}^{Q}=\Gamma_{Q} \times K_{Q}$.

For $\gamma \in \Gamma W$ with $\gamma(Q)=Q^{\prime} \subset \Delta$ there are algebra isomorphisms

$$
\begin{aligned}
\psi_{\gamma}: \mathcal{H}_{Q} \rightarrow \mathcal{H}_{Q^{\prime}}, \quad \theta_{x_{Q}} N_{w} & \mapsto \theta_{\gamma\left(x_{Q}\right)} N_{\gamma w \gamma^{-1}}, \\
\psi_{\gamma}: \mathcal{H}^{Q} \rightarrow \mathcal{H}^{Q^{\prime}}, \quad \theta_{x} N_{w} & \mapsto \theta_{\gamma x} N_{\gamma w \gamma^{-1}} .
\end{aligned}
$$

The groupoid $\mathcal{G}$ acts from the left on $\Xi_{\text {un }}$ by

$$
(g, u) \cdot(Q, \delta, t):=\left(g(Q), \delta \circ \psi_{u}^{-1} \circ \psi_{g}^{-1}, g(u t)\right),
$$

the action being defined if and only if $g(Q) \subset \Delta$.

Suppose that $g(Q)=Q^{\prime} \subset \Delta$ and $\delta^{\prime} \cong \delta \circ \psi_{u}^{-1} \circ \psi_{g}^{-1}$. By [Opd, Theorem 4.33] and [Sol3, Theorem 3.1.5] there exists an intertwining operator

$$
\pi^{\Gamma}(g u, Q, \delta, t) \in \operatorname{Hom}_{\mathcal{H}(\mathcal{R}, q) \rtimes \Gamma}\left(\pi^{\Gamma}(Q, \delta, t), \pi^{\Gamma}\left(Q^{\prime}, \delta^{\prime}, g(u t)\right)\right),
$$

which depends algebraically on $t \in T_{\mathrm{un}}^{Q}$. This implies that, for all $\xi \in \Xi$ and $g \in \mathcal{G}$ such that $g \xi$ is defined, $\pi^{\Gamma}(\xi)$ and $\pi^{\Gamma}(g \xi)$ have the same irreducible constituents, counted with multiplicity [Sol3, Lemma 3.1.7]. 
The action of $\mathcal{G}$ on the continuous sections $C\left(\Xi_{\mathrm{un}} ; \operatorname{End}\left(\mathcal{V}_{\Xi}^{\Gamma}\right)\right)$ is given by

$$
(g \cdot f)(g \xi)=\pi^{\Gamma}(g, \xi) f(\xi) \pi^{\Gamma}(g, \xi)^{-1} \quad g \in \mathcal{G}_{Q Q}, \xi=(Q, \delta, t) .
$$

The next result is the Plancherel isomorphism for affine Hecke algebras, proven in [DeOp, Theorem 5.3 and Corollary 5.7] and [Sol3, Theorem 3.2.2].

Theorem 1.4. The map Fourier transform (18) induces *-homomorphisms

$$
\begin{aligned}
\mathcal{H}(\mathcal{R}, q) \rtimes \Gamma & \rightarrow & \mathcal{O}\left(\Xi ; \operatorname{End}\left(\mathcal{V}_{\Xi}^{\Gamma}\right)\right)^{\mathcal{G}}, \\
\mathcal{S}(\mathcal{R}, q) \rtimes \Gamma & \rightarrow & C^{\infty}\left(\Xi_{\text {un }} ; \operatorname{End}(\mathcal{V} \Xi)\right)^{\mathcal{G}}, \\
C_{r}^{*}(\mathcal{R}, q) \rtimes \Gamma & \rightarrow & C\left(\Xi_{\text {un }} ; \operatorname{End}\left(\mathcal{V}_{\Xi}^{\Gamma}\right)\right)^{\mathcal{G}} .
\end{aligned}
$$

The first is injective, the second is an isomorphism of Fréchet algebras and the third is an isomorphism of $C^{*}$-algebras.

For $q=1$ these simplify to the well-known isomorphisms

$$
\begin{array}{rrrrr}
\mathcal{H}(\mathcal{R}, 1) \rtimes \Gamma & = & \mathcal{O}(T) \rtimes W \Gamma & \rightarrow & \mathcal{O}\left(T ; \operatorname{End}_{\mathbb{C}}(\mathbb{C}[W \Gamma])\right)^{W \Gamma}, \\
\mathcal{S}(\mathcal{R}, 1) \rtimes \Gamma & = & C^{\infty}\left(T_{\text {un }}\right) \rtimes W \Gamma & \rightarrow & C^{\infty}\left(T_{\text {un }} ; \operatorname{End}_{\mathbb{C}}(\mathbb{C}[W \Gamma])\right)^{W \Gamma}, \\
C_{r}^{*}(\mathcal{R}, 1) \rtimes \Gamma & = & C\left(T_{\text {un }}\right) \rtimes W \Gamma & \rightarrow & C\left(T_{\text {un }} ; \operatorname{End}_{\mathbb{C}}(\mathbb{C}[W \Gamma])\right)^{W \Gamma} .
\end{array}
$$

Unfortunately, the bookkeeping in Theorem 1.4 is not entirely suitable for our purposes, because sometimes the parabolic subalgebras need to be extended by diagram automorphisms. In those cases we should rather use induction data based on $\operatorname{Irr}_{L^{2}}\left(\mathcal{H}^{Q} \rtimes \Gamma_{Q}\right)$ than based on $\operatorname{Irr}_{L^{2}}\left(\mathcal{H}_{Q}\right)$ or $\operatorname{Irr}_{L^{2}}\left(\mathcal{H}^{Q}\right)$.

We fix a system of subgroups $\Gamma_{Q} \subset \Gamma(Q \subset \Delta)$ satisfying Condition 1.1. With Lemma 1.3 in mind we define new induction data. They are triples $(Q, \sigma, t)$ with $Q \subset \Delta, t \in T^{Q}$ and $\sigma \in \operatorname{Irr}_{L^{2}}\left(\mathcal{H}_{Q} \rtimes \Gamma_{Q}\right)$. We regard another such triple $\left(Q^{\prime}, \sigma^{\prime}, t^{\prime}\right)$ as equivalent if and only if $Q^{\prime}=Q, t^{\prime}=t$ and $\sigma^{\prime} \cong \sigma$. We keep the same groupoid $\mathcal{G}$ as before, it also acts on the new triples via (24). To such a triple we associate the representation

$$
\pi(Q, \sigma, t)=\operatorname{ind}_{\mathcal{H}^{Q} \rtimes \Gamma_{Q}}^{\mathcal{H} \rtimes \Gamma}(\sigma \otimes t) .
$$

The vector space underlying $\pi(Q, \sigma, t)$ does not depend on $t$, we denote it by $V_{Q, \sigma}$. There is a natural homomorphism

$$
\begin{array}{cl}
\mathcal{H}(\mathcal{R}, q) \rtimes \Gamma & \rightarrow \mathcal{O}\left(T^{Q}\right) \otimes \operatorname{End}_{\mathbb{C}}\left(V_{Q, \sigma}\right) \\
h & \mapsto(t \mapsto \pi(Q, \sigma, t)(h)) .
\end{array}
$$

We refer to the system of these maps, for all $Q$ and $\sigma$, as the Fourier transform for $\mathcal{H}(\mathcal{R}, q) \rtimes \Gamma$. The recipe for the intertwining operators from [Opd, §4] and Sol3, Theorem 3.1.5] remains valid, so we get

$$
\pi(g u, Q, \sigma, t) \in \operatorname{Hom}_{\mathcal{H} \rtimes \Gamma}\left(\pi(Q, \sigma, t), \pi\left(g(Q), \sigma^{\prime}, g(u t)\right)\right)
$$

with the same properties as in (25). In particular $\pi(Q, \sigma, t)$ and $\pi\left(g(Q), \sigma^{\prime}, g(u t)\right)$ have the same irreducible constituents, counted with multiplicity. With these notions we can vary on the Plancherel isomorphism (Theorem 1.4).

To do so, we first consider essentially discrete series representations of $\mathcal{H}^{Q} \rtimes \Gamma_{Q}$. Pick $\delta_{1} \in \operatorname{Irr}_{L^{2}}\left(\mathcal{H}_{Q}\right)$ and $t_{1} \in T_{\mathrm{un}}^{Q}$. We note that $\operatorname{ind}_{\mathcal{H}^{Q}}^{\mathcal{H}^{Q} \rtimes \Gamma_{Q}}\left(\delta \otimes t_{1}\right)$ is unitary and 
essentially discrete series, because $\Gamma_{Q}$ stabilizes $Q$. Write $\mathcal{G}_{Q Q}^{Q} \delta_{1}=\left\{\delta_{i}\right\}_{i}$. The summand of $C^{\infty}\left(\Xi_{Q, \text { un }} ; \operatorname{End}\left(\mathcal{V}_{\Xi_{Q}}^{\Gamma_{Q}}\right)\right)^{\mathcal{G}^{Q}}$ associated to $\left(Q, \delta_{1}\right)$ is

$$
\left(\bigoplus_{i} C^{\infty}\left(T_{\mathrm{un}}^{Q} ; \operatorname{End}_{\mathbb{C}}\left(\pi^{\Gamma_{Q}}\left(Q, \delta_{i}, t_{i}\right)\right)\right)\right)^{\mathcal{G}_{Q Q}^{Q}} .
$$

Let $\left\{\sigma_{j}\right\}_{j}$ be the members of $\operatorname{Irr}_{L^{2}}\left(\mathcal{H}_{Q} \rtimes \Gamma_{Q}\right)$ contained in $\operatorname{ind}_{\mathcal{H}_{Q}}^{\mathcal{H}_{Q} \rtimes \Gamma_{Q}}\left(\delta_{1} \circ \psi_{u}\right)$ for some $u \in K_{Q}$. This set is stable under $\mathcal{G}_{Q Q}^{Q}=\Gamma_{Q} \times K_{Q}$. The summand of $\left(\bigoplus_{\sigma} C^{\infty}\left(T_{\mathrm{un}}^{Q} ; \operatorname{End}_{\mathbb{C}}\left(V_{\sigma}\right)\right)\right)^{\mathcal{G}^{Q}}$ corresponding to the $\sigma_{j}$ is

$$
\left(\bigoplus_{j} C^{\infty}\left(T_{\mathrm{un}}^{Q} ; \operatorname{End}_{\mathbb{C}}\left(V_{\sigma_{j}}\right)\right)\right)^{\mathcal{G}_{Q Q}^{Q}}
$$

Lemma 1.5. The algebras (31) and (32) are naturally isomorphic.

Proof. For $\sigma \in \operatorname{Irr}_{L^{2}}\left(\mathcal{H}^{Q} \rtimes \Gamma_{Q}\right)$ we write $(\sigma, t)>\left(\delta_{1}, t_{1}\right)$ if

$$
\operatorname{Hom}_{\mathcal{H}^{Q} \rtimes \Gamma_{Q}}\left(\sigma \otimes t, \operatorname{ind}_{\mathcal{H}^{Q}}^{\mathcal{H}^{Q} \rtimes \Gamma_{Q}}\left(\delta \otimes t_{1}\right)\right) \cong \operatorname{Hom}_{\mathcal{H}^{Q}}\left(\sigma \otimes t, \delta \otimes t_{1}\right)
$$

is nonzero. Since $\Gamma_{Q}$ is finite, the set of such $(\sigma, t)$ is finite. Hence the map

$$
\bigoplus_{(\sigma, t)>\left(\delta_{1}, t_{1}\right)} \sigma \circ \psi_{t}: \mathcal{H}^{Q} \rtimes \Gamma_{Q} \rightarrow \bigoplus_{(\sigma, t)>\left(\delta_{1}, t_{1}\right)} \operatorname{End}_{\mathbb{C}}\left(V_{\sigma}\right)
$$

is surjective. The specialization of (31) at $\mathcal{G}_{Q Q}^{Q}\left(Q, \delta_{1}, t_{1}\right)$ is also $\bigoplus_{(\sigma, t)>\left(\delta_{1}, t_{1}\right)} \operatorname{End}_{\mathbb{C}}\left(V_{\sigma}\right)$, for that specialization is just

$$
\operatorname{ind}_{\mathcal{H}^{Q}}^{\mathcal{H}^{Q} \rtimes \Gamma_{Q}}\left(\delta \circ \psi_{t_{1}}\right)\left(\mathcal{S}\left(\mathcal{R}^{Q}, q^{Q}\right) \rtimes \Gamma_{Q}\right) .
$$

Similarly, specializing the algebra (32) at all $(\sigma, t)>\left(\delta_{1}, t_{1}\right)$ gives a surjection from (32) to $\bigoplus_{(\sigma, t)>\left(\delta_{1}, t_{1}\right)} \operatorname{End}_{\mathbb{C}}\left(V_{\sigma}\right)$.

Now we can explicitly compare (32) with (31). Both are algebras of smooth sections of (trivial) algebra bundles, and specialization at the points associated to $\left(\delta_{1}, t_{1}\right)$ yields the same algebra in both cases. This holds for any $t_{1} \in T_{\mathrm{un}}^{Q}$ and that accounts for all base points of these algebra bundles, so (31) and (32) are isomorphic. Moreover the isomorphism is canonical: it is the composition of the inverse of the map in Theorem 1.4 and the map induced by (29) (both for $\mathcal{H}^{Q} \rtimes \Gamma_{Q}$ ).

Next we formulate our variation on the Plancherel isomorphism.

Proposition 1.6. The Fourier transform from (29) induces isomorphisms of Fréchet *algebras

$$
\begin{aligned}
\mathcal{S}(\mathcal{R}, q) \rtimes \Gamma & \rightarrow\left(\bigoplus_{Q, \sigma} C^{\infty}\left(T_{\mathrm{un}}^{Q} ; \operatorname{End}_{\mathbb{C}}\left(V_{Q, \sigma}\right)\right)\right)_{\mathcal{G}}^{\mathcal{G}}, \\
C_{r}^{*}(\mathcal{R}, q) \rtimes \Gamma & \rightarrow\left(\bigoplus_{Q, \sigma} C\left(T_{\mathrm{un}}^{Q} ; \operatorname{End}_{\mathbb{C}}\left(V_{Q, \sigma}\right)\right)\right)^{\mathcal{G}} .
\end{aligned}
$$

Proof. We will analyse the right hand side of Theorem1.4(for the Schwartz algebras) and compare it with the current setting. 
For every $Q \subset \Delta$, Lemma 1.5 yields a canonical isomorphism

$$
\begin{aligned}
\left(\bigoplus_{\sigma \in \operatorname{Irr}_{L^{2}}\left(\mathcal{H}_{Q} \rtimes \Gamma_{Q}\right)} C^{\infty}\left(T_{\mathrm{un}}^{Q} ; \operatorname{End}_{\mathbb{C}}\left(V_{\sigma}\right)\right)\right)^{\mathcal{G}_{Q Q}^{Q}} \cong \\
\left(\bigoplus_{\delta \in \operatorname{Irr}_{L^{2}}\left(\mathcal{H}_{Q}\right)} C^{\infty}\left(T_{\mathrm{un}}^{Q} ; \operatorname{End}_{\mathbb{C}}\left(\mathbb{C}\left[\Gamma_{Q}\right] \otimes V_{\delta}\right)\right)\right)^{\mathcal{G}_{Q Q}^{Q}} .
\end{aligned}
$$

To obtain the right hand side of Theorem 1.4 from (33), we must apply $\operatorname{ind}_{\mathcal{H}^{Q} \rtimes \Gamma_{Q}}^{\mathcal{H} \rtimes \Gamma}$ to $\mathbb{C}\left[\Gamma_{Q}\right] \otimes V_{\delta} \cong \operatorname{ind}_{\mathcal{H}^{Q}}^{\mathcal{H}^{Q} \Gamma_{Q}}(\delta \otimes t)$ and then take invariants with respect to the larger groupoid $\mathcal{G} \supset \mathcal{G}^{Q}$. The formula $\left[\right.$ Sol3, (3.12)] is the same for $\mathcal{G}^{Q}$ and for $\mathcal{G}$, so the intertwiners associated to elements of $\mathcal{G}^{Q}$ need not be adjusted in this process.

With exactly the same procedure we can turn (33) into the right hand side of the current proposition. The intertwining operators associated to elements of $\mathcal{G}$ agree under the isomorphisms obtained from (33) by applying $\operatorname{ind}_{\mathcal{H}^{Q} \rtimes \Gamma_{Q}}^{\mathcal{H} \rtimes \Gamma}$, because in both settings they were constructed with [Sol3, (3.12) and Theorem 3.1.5]. Consequently

$$
\left(\bigoplus_{Q, \sigma} C^{\infty}\left(T_{\mathrm{un}}^{Q} ; \operatorname{End}_{\mathbb{C}}\left(V_{Q, \sigma}\right)\right)\right)^{\mathcal{G}} \cong C^{\infty}\left(\Xi_{\mathrm{un}} ; \operatorname{End}\left(\mathcal{V}_{\Xi}^{\Gamma}\right)\right)^{\mathcal{G}}
$$

proving the proposition for the Schwartz algebras. For $C_{r}^{*}(\mathcal{R}, q) \rtimes \Gamma$ one can use the same argument, with everywhere $C^{\infty}$ replaced by continuous functions.

Choose representatives $Q$ for $\mathcal{P}(\Delta)$ modulo $W \Gamma$-association. For every such $Q$ we choose representatives $\sigma$ for the action of $\mathcal{G}_{Q Q}=W \Gamma(Q, Q) \times K_{Q}$ on $\operatorname{Irr}_{L^{2}}\left(\mathcal{H}_{Q} \rtimes \Gamma_{Q}\right)$. By Lemma 1.3 these $\sigma$ also form representatives for the action of $\mathcal{G}_{Q Q} \ltimes T_{\text {un }}^{Q}$ on $\operatorname{Irr}_{L^{2}}\left(\mathcal{H}^{Q} \rtimes \Gamma_{Q}\right)$. We denote the resulting set of representatives of pairs by $(Q, \sigma) / \mathcal{G}$. Let $\mathcal{G}_{Q, \sigma}$ be the setwise stabilizer of $\left(Q, \sigma, T_{\mathrm{un}}^{Q}\right)$ in the group $\mathcal{G}_{Q Q}$. Proposition [1.6 can be rephrased as isomorphisms

$$
\begin{gathered}
\mathcal{S}(\mathcal{R}, q) \rtimes \Gamma \rightarrow \bigoplus_{(Q, \sigma) / \mathcal{G}} C^{\infty}\left(T_{\mathrm{un}}^{Q} ; \operatorname{End}_{\mathbb{C}}\left(V_{Q, \sigma}\right)\right)^{\mathcal{G}_{Q, \sigma},}, \\
C_{r}^{*}(\mathcal{R}, q) \rtimes \Gamma \rightarrow \bigoplus_{(Q, \sigma) / \mathcal{G}} C\left(T_{\mathrm{un}}^{Q} ; \operatorname{End}_{\mathbb{C}}\left(V_{Q, \sigma}\right)\right)^{\mathcal{G}_{Q, \sigma}} .
\end{gathered}
$$

Sometimes we have to consider the opposite algebra $(\mathcal{H}(\mathcal{R}, q) \rtimes \Gamma)^{\text {op }}$ and its completions. It is, morally, clear that all the previous results can also developed for right $\mathcal{H} \rtimes \Gamma$-modules, that is, for $(\mathcal{H} \rtimes \Gamma)^{\mathrm{op}}$-modules. However, none of that has been written down, so we prefer more steady ground.

For every $\mathcal{H} \rtimes \Gamma$-representation $\left(\pi, V_{\pi}\right)$, the full linear dual $V_{\pi}^{*}$ becomes a $(\mathcal{H} \rtimes \Gamma)^{\mathrm{op}}$ representation $\pi^{*}$ by

$$
\pi^{*}\left(h^{\mathrm{op}}\right) \lambda=\lambda \circ \pi(h) .
$$

This sets up a bijection between finite dimensional left and right modules of $\mathcal{H} \rtimes \Gamma$. In view of the canonical inner products from on the spaces (19), this bijection commutes with induction from parabolic subalgebras.

For infinite dimensional representations there is often some choice for which dual space of $V_{\pi}$ we use here. In particular, when $V_{\pi}$ is a Hilbert space we can use $V_{\pi}$ also as dual space. With this convention one checks easily that $\pi$ is unitary if and only if $\pi^{*}$ is unitary.

The $\mathcal{O}(T)$-weights of $\pi^{*}$ are the same as for $\pi$, so $\pi^{*}$ is tempered or (essentially) discrete series if and only if $\pi$ is so. Thus the pairs $(Q, \sigma)$ with $\sigma \in \operatorname{Irr}_{L^{2}}\left(\mathcal{H}_{Q} \rtimes \Gamma_{Q}\right)$ 
are in natural bijection with the pairs $\left(Q, \sigma^{*}\right)$ in

$$
\bigcup_{Q \subset \Delta} \operatorname{Irr}_{L^{2}}\left(\left(\mathcal{H}\left(\mathcal{R}_{Q}, q_{Q}\right) \rtimes \Gamma_{Q}\right)^{\mathrm{op}}\right) .
$$

The bijection is $\mathcal{G}$-equivariant for the $\mathcal{G}$-action on (35) as in (24). Hence $\mathcal{G}_{Q, \sigma}=\mathcal{G}_{Q, \sigma^{*}}$ and we can take $\left(Q, \sigma^{*}\right) / \mathcal{G}$ to be the image of $(Q, \sigma) / \mathcal{G}$.

Lemma 1.7. The Fourier transform for right $\mathcal{H}(\mathcal{R}, q) \rtimes \Gamma$-modules induces isomorphisms of Fréchet *algebras

$$
\begin{array}{cl}
(\mathcal{S}(\mathcal{R}, q) \rtimes \Gamma)^{\mathrm{op}} & \rightarrow \bigoplus_{\left(Q, \sigma^{*}\right) / \mathcal{G}} C^{\infty}\left(T_{\mathrm{un}}^{Q} ; \operatorname{End}_{\mathbb{C}}\left(V_{Q, \sigma^{*}}\right)\right)^{\mathcal{G}_{Q, \sigma^{*}}} \\
\left(C_{r}^{*}(\mathcal{R}, q) \rtimes \Gamma\right)^{\mathrm{op}} & \rightarrow \bigoplus_{\left(Q, \sigma^{*}\right) / \mathcal{G}} C\left(T_{\mathrm{un}}^{Q} ; \operatorname{End}_{\mathbb{C}}\left(V_{Q, \sigma^{*}}\right)\right)^{\mathcal{G}_{Q, \sigma^{*}}}
\end{array}
$$

Proof. The opposite algebra of

$$
\operatorname{End}_{\mathbb{C}}\left(V_{Q, \sigma}\right)=\operatorname{End}_{\mathbb{C}}\left(\operatorname{ind}_{\mathcal{H}^{Q} \rtimes \Gamma_{Q}}^{\mathcal{H} \rtimes \Gamma} V_{\delta}\right)
$$

is naturally isomorphic to $\operatorname{End}_{\mathbb{C}}\left(V_{Q, \sigma}^{*}\right)$, which by $\mathrm{Opd}$, Proposition 4.19] is canonically isomorphic with

$$
\operatorname{End}_{\mathbb{C}}\left(\operatorname{ind}_{\mathcal{H}^{Q} \rtimes \Gamma_{Q}}^{\mathcal{H} \rtimes \Gamma}\left(V_{\sigma}^{*}\right)\right)=\operatorname{End}_{\mathbb{C}}\left(V_{Q, \sigma^{*}}\right) .
$$

For $g \in \mathcal{G}_{Q, \sigma}$ we take $\pi\left(g, Q, \sigma^{*}, t|t|^{-2}\right)$ to be the transpose inverse of $\pi(g, Q, \sigma, t)$. Thus an element of $C\left(T_{\mathrm{un}}^{Q} ; \operatorname{End}_{\mathbb{C}}\left(V_{Q, \sigma}\right)\right)$ is $\mathcal{G}_{Q, \sigma}$-invariant if and only if its transpose in $C\left(T_{\mathrm{un}}^{Q} ; \operatorname{End}_{\mathbb{C}}\left(V_{Q, \sigma^{*}}\right)\right)$ is $\mathcal{G}_{Q, \sigma^{*}}$-invariant for the action

$$
(g \cdot f)\left(g\left(Q, \sigma^{*}, t\right)\right)=\pi\left(g, Q, \sigma^{*}, t\right) f\left(Q, \sigma^{*}, t\right) \pi\left(g, Q, \sigma^{*}, t\right)^{-1} .
$$

Now we take the opposite algebras in Theorem 1.4 and we find the desired isomorphisms.

The implementing algebra homomorphisms are given by transpose, the Fourier transform from (29) and again transpose, which works out to the Fourier transform for $(\mathcal{H}(\mathcal{R}, q) \rtimes \Gamma)^{\mathrm{op}}$-modules. Since the correspondence between left and right $\mathcal{H}(\mathcal{R}, q) \rtimes \Gamma$-modules preserves unitarity, the latter Fourier transform is still a *_ homomorphism.

\subsection{The space of irreducible representations.}

We compare the irreducible representations of $\mathcal{H} \rtimes \Gamma$ to its representations induced from proper parabolic subalgebras (i.e. the algebras $\mathcal{H}^{Q} \rtimes \Gamma_{Q}$ with $Q \subsetneq \Delta$ ). Let $\operatorname{Gr}(\mathcal{H} \rtimes \Gamma)$ be the Grothendieck group of the category of finite length $\mathcal{H} \rtimes \Gamma$ representations and write $\operatorname{Gr}_{\mathbb{Q}}(\mathcal{H} \rtimes \Gamma)=\mathbb{Q} \otimes_{\mathbb{Z}} \operatorname{Gr}(\mathcal{H} \rtimes \Gamma)$. Then (parabolic) induction induces a $\mathbb{Q}$-linear map $\operatorname{Gr}_{\mathbb{Q}}\left(\mathcal{H}_{Q} \rtimes \Gamma_{Q}\right) \rightarrow \operatorname{Gr}_{\mathbb{Q}}(\mathcal{H} \rtimes \Gamma)$.

Theorem 1.8. (a) The collection of irreducible $\mathcal{H} \rtimes \Gamma$-representations whose image in $\operatorname{Gr}_{\mathbb{Q}}(\mathcal{H} \rtimes \Gamma)$ is not a $\mathbb{Q}$-linear combination of representations induced from proper parabolic subalgebras is a nonempty union of $T^{W \Gamma}$-orbits in $\operatorname{Irr}(\mathcal{H} \rtimes \Gamma)$.

(b) Suppose that, for every $w \in W \Gamma \backslash \bigcup_{Q \subsetneq \Delta} W\left(R_{Q}\right) \Gamma_{Q}$, the set $T_{\Delta}^{w}$ is finite. Then the set in part (a) is a finite union of $T^{W \Gamma}$-orbits.

Proof. (a) Recall from (13) that $\mathcal{O}(T)^{W\left(R_{Q}\right) \Gamma_{Q}} \subset Z\left(\mathcal{H}^{Q} \rtimes \Gamma_{Q}\right)$. Hence all irreducible representations of $\mathcal{H}^{Q} \rtimes \Gamma_{Q}$ come in families parametrized by $T^{W\left(R_{Q}\right) \Gamma_{Q}}$. Since $T^{W \Gamma} \subset T^{W\left(R_{Q}\right) \Gamma_{Q}}$ for all $Q \subset \Delta$, the set of irreducible representations under consideration is a union of $T^{W \Gamma}$-orbits. 
By [Sol4, Lemma 2.3], $\operatorname{Irr}(\mathcal{H} \rtimes \Gamma)$ can be parametrized by the extended quotient

$$
T / / W \Gamma=\left(\bigcup_{w \in W \Gamma}\{w\} \times T^{w}\right) / W \Gamma
$$

where $W \Gamma$ acts on the union by $w^{\prime} \cdot(w, t)=\left(w^{\prime} w w^{-1}, w^{\prime}(t)\right)$. This parametrization respects central characters, up to a twists which are constant on connected components of $T / / W \Gamma$ [Sol4, Theorem 2.6]. In this parametrization of $\operatorname{Irr}(\mathcal{H} \rtimes \Gamma)$ almost all elements of a piece $\{w\} \times T^{w}$ with $w \in W\left(R_{Q}\right) \Gamma_{Q}$ come from representations induced from $\mathcal{H}^{Q} \rtimes \Gamma_{Q}$, and such $w$ account for all representations induced from proper parabolic subalgebras. Hence the set considered in the statement can be parametrized by

$$
\left(\bigcup_{w \in W \Gamma: w \notin W\left(R_{Q}\right) \Gamma_{Q} \forall Q \subsetneq \Delta}\{w\} \times T^{w}\right) / W \Gamma
$$

This set is nonempty because every Coxeter element of $W=W(R)$ contributes at least $(w, 1)$ to it.

(b) This is obvious from (36).

The induction data from $\Xi$ give rise to a partition $\operatorname{Irr}(\mathcal{H} \rtimes \Gamma)$ into finite packets.

Theorem 1.9. [Sol3, Theorem 3.3.2.b]

For every $\pi \in \operatorname{Irr}(\mathcal{H} \rtimes \Gamma)$ there exists a unique $\mathcal{G}$-association class $\mathcal{G}(Q, \delta, t) \in \Xi / \mathcal{G}$ such that $\pi$ is a constituent of $\pi^{\Gamma}(Q, \delta, t)$ and the invariant $\|c c(\delta)\|$ from (14) is maximal for this property.

With the new induction data $(Q, \sigma, t)$ from (28) we can vary on Theorem 1.9. (Now $\sigma \in \operatorname{Irr}_{L^{2}}\left(\mathcal{H}_{Q} \rtimes \Gamma_{Q}\right)$, whereas the above $\delta$ was a representation of $\mathcal{H}_{Q}$. )

Theorem 1.10. (a) For every $\pi \in \operatorname{Irr}(\mathcal{H} \rtimes \Gamma)$ there exists a triple $(Q, \sigma, t)$ as above, such that $\pi$ is a constituent of $\pi(Q, \sigma, t)$ and $\|c c(\sigma)\|$ is maximal for this property. In this situation we say that $\pi$ is a Langlands constituent of $\pi(Q, \sigma, t)$.

(b) In the setting of part (a), the restriction of $\sigma \otimes t$ to $\mathcal{H}^{Q}$ is a direct sum of irreducible representations in one $\Gamma_{Q^{-o r b i t}}$, say $\Gamma_{Q}(\delta \otimes t) \subset \operatorname{Irr}_{L^{2}}\left(\mathcal{H}^{Q}\right)$. Then $(Q, \delta, t)$ is uniquely determined by $\pi$, up to the action of $\mathcal{G}$.

(c) Let $(Q, \sigma, t)$ be any induction datum as in (28). Every constituent of $\pi(Q, \sigma, t)$ is either a Langlands constituent or a constituent of $\pi\left(Q^{\prime}, \sigma^{\prime}, t^{\prime}\right)$ for some induction datum with $\left\|c c\left(\sigma^{\prime}\right)\right\|>\|c c(\sigma)\|$.

(d) $\pi$ is tempered if and only if $t \in T_{\mathrm{un}}$, where $t \in T^{Q}$ comes from part (a).

Proof. (a) Let $(Q, \delta, t)$ be as in Theorem 1.9. Thus $\pi$ is a constituent of

$$
\pi^{\Gamma}(Q, \delta, t)=\operatorname{ind}_{\mathcal{H}^{Q} \rtimes \Gamma_{Q}}^{\mathcal{H} \rtimes \Gamma}\left(\operatorname{ind}_{\mathcal{H}^{Q}}^{\mathcal{H}^{Q} \rtimes \Gamma_{Q}}(\delta \otimes t)\right),
$$

and the norm of the central character of $\delta$ is maximal for this property. Let $T^{--Q}$ be the subset $T^{--}$of $T$, but computed with respect to $Q$. Every $\mathcal{A}$-weight of $\operatorname{ind}_{\mathcal{H}^{Q}}^{\mathcal{H}^{Q} \rtimes \Gamma_{Q}}(\delta \otimes t)$ lies in one of the $\Gamma_{Q^{-}}$orbits of weights of $\delta \otimes t$, which are entirely contained in $T^{--Q} T_{\text {un }}$ because $\Gamma_{Q}$ stabilizes $Q$. In other words, $\operatorname{ind}_{\mathcal{H}^{Q}}^{\mathcal{H}^{Q} \rtimes \Gamma_{Q}}(\delta \otimes t)$ is a direct sum of finitely many irreducible essentially discrete series representations of $\mathcal{H}^{Q} \rtimes \Gamma$, all with central characters in the same $W_{Q} \rtimes \Gamma_{Q}$-orbit. By Lemma 1.3 all these summands are of the form $\sigma_{i} \otimes t$ with $\sigma_{i} \in \operatorname{Irr}_{L^{2}}\left(\mathcal{H}_{Q} \rtimes \Gamma_{Q}\right)$. Hence

$$
\pi^{\Gamma}(Q, \delta, t)=\bigoplus_{i} \pi\left(Q, \sigma_{i}, t\right)
$$


and $\pi$ is a constituent of (at least) one $\pi\left(Q, \sigma_{i}, t\right)$.

The central characters of the $\sigma_{i}$ and of $\delta$ have the same norm, and that of $\delta$ was maximal given $\pi$. Hence the norm of the central character of $\sigma_{i}$ is also maximal, given $\pi$.

(b) Suppose that $(Q, \sigma, t)$ satisfies the requirements of part (a), that is, $\pi$ is a Langlands constituent of $\pi(Q, \sigma, t)$. In the proof of Lemma 1.3 we observed that the restriction of $\sigma \otimes t$ to $\mathcal{H}^{Q}$ is a direct sum of representations of the form $\gamma\left(\left(\delta \otimes k^{-1}\right) \otimes k t\right)$ with $\gamma \in \Gamma_{Q}$ and $k \in K_{Q}$. For all $\gamma \in \Gamma_{Q}, \pi$ is a constituent of

$$
\pi^{\Gamma}(Q, \delta, t)=\pi^{\Gamma}\left(Q, \gamma\left(\delta \otimes k^{-1}\right), \gamma(k t)\right) .
$$

The norm of the central character of $\gamma\left(\delta \otimes k^{-1}\right)$ is maximal for this property, by the assumption on $\sigma$. By Theorem [1.9] all $\left(Q, \gamma\left(\delta \otimes k^{-1}\right), \gamma(k t)\right)$ lie in a unique $\mathcal{G}$-association class in $\Xi$ determined by $\pi$.

(c) In view of (37), it suffices to consider the constituents of $\pi^{\Gamma}(Q, \delta, t)$. Then the statement is implicit in [Sol3], we make it more explicit here. By Sol3, Lemma 3.1.7 and Theorem 3.3.2], we may furthermore assume that $\xi=(Q, \delta, t)$ is in positive position, that is, $|t| \in T^{Q+}=\exp \left(\mathfrak{a}^{Q} \cap \mathfrak{a}^{+}\right)$. Write $P(\xi)=\{\alpha \in \Delta:|\alpha(t)|=1\}$

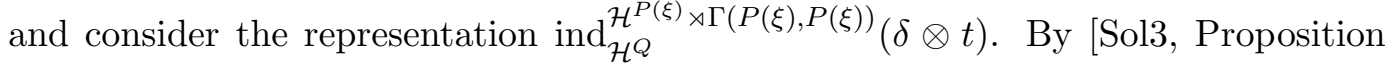
3.1.4] that representation is completely reducible, and all its irreducible summands are of the form $\delta^{\prime} \otimes t^{\prime}$ where $\delta^{\prime} \in \operatorname{Irr}\left(\mathcal{H}_{P(\xi)} \rtimes \Gamma(P(\xi), P(\xi))\right)$ is tempered and $t^{\prime} \in$ $T^{P(\xi)} \cap t T_{P(\xi)}$ with

$$
\left|t^{\prime}\right| \in T^{P(\xi)++}=\exp \left(\left\{\mu \in \mathfrak{a}^{P(\xi)}:\langle\alpha, \mu\rangle>0 \forall \alpha \in \Delta \backslash P(\xi)\right\}\right) .
$$

Thus every constituent $\pi(Q, \sigma, t)$ is a constituent of

$$
\operatorname{ind}_{\mathcal{H}^{P(\xi)} \rtimes \Gamma(P(\xi), P(\xi))}^{\mathcal{H} \rtimes \Gamma}\left(\delta^{\prime} \otimes t^{\prime}\right)
$$

for such a $\delta^{\prime} \otimes t^{\prime}$. By [Sol3, Theorem 3.3.2], the Langlands constituents of $\pi(Q, \sigma, t)$ (or $\left.\pi^{\Gamma}(Q, \delta, t)\right)$ are precisely those constitutents which are quotients of a representation (38). The Langlands classification for extended affine Hecke algebras [Sol3, Corollary 2.2.5], says that the latter representation has a unique irreducible quotient (called the Langlands quotient, hence our terminology Langlands constituents). Moreover by [Sol3, Lemma 2.2.6.b] all other constituents of (38) are Langlands quotients for data where the norm of the central character is bigger than $\|c c(\sigma)\|$. Then [Sol3, Proposition 3.1.4 and Theorem 3.3.2] entail that those other irreducible representations occur as Langlands constituents of a $\pi^{\Gamma}\left(Q^{\prime}, \delta^{\prime}, t^{\prime}\right)$ with $\left\|c c\left(\delta^{\prime}\right)\right\|>\|c c(\sigma)\|=\|c c(\delta)\|$.

(d) As observed in the proof of part (c), in the construction for part (a) and for Sol3, Theorem 3.3.2] we may assume that $(Q, \sigma, t)$ is positive and that $\pi$ is the unique Langlands quotient of (38), for one of the above $\delta^{\prime} \otimes t^{\prime}$. By the uniqueness in the Langlands classification for extended affine Hecke algebras [Sol3, Corollary 2.2.5], $\pi$ is tempered if and only if $P(\xi)=\Delta$ and $t^{\prime} \in T_{\text {un }}^{\Delta}$.

Here $P(\xi)=\Delta$ implies $|t|=\left|t^{\prime}\right| \in T^{\Delta}$, and then $t^{\prime} \in T_{\text {un }}^{\Delta}$ says that $|t|=1$. Conversely, $t \in T_{\text {un }}$ implies $P(\xi)=\Delta$ and $t=t^{\prime} \in T^{\Delta}$.

With Theorem 1.10 one can express the structure of $\operatorname{Irr}(\mathcal{H} \rtimes \Gamma)$ in terms of its subset of irreducible tempered representations. In essence, the former is the complexification of the latter (which is something like a real algebraic variety with multiplicities). We do not need the full strength of this. For our purposes it suffices to 
consider half-lines in the parameter space, such that $Q, \sigma$ and the unitary part of $t$ are fixed and the absolute value of $t$ can be scaled by a positive factor.

Proposition 1.11. Let $(Q, \sigma, t)$ be an induction datum with $\sigma \in \operatorname{Irr}_{L^{2}}\left(\mathcal{H}^{Q} \rtimes \Gamma_{Q}\right)$.

(a) For $r \in \mathbb{R}_{>-1}$, the number of inequivalent Langlands constituents of $\pi\left(Q, \sigma, t|t|^{r}\right)$ does not depend on $r$.

(b) For all but finitely many $r \in \mathbb{R}_{>-1}, \pi\left(Q, \sigma, t|t|^{r}\right)$ is completely reducible. Then all its irreducible subquotients are Langlands constituents.

Proof. (a) Let $\xi=(Q, \delta, t) \in \Xi$ be as in Theorem 1.10.

Notice that the intertwining operators $\pi(g u, Q, \sigma, t)$ depend algebraically on $t \in$ $T^{Q}$. This implies that, for every $g u \in \mathcal{G}, \pi(Q, \sigma, t)$ and $\pi(g u(Q, \sigma, t))$ have the same irreducible subquotients (counted with multiplicity), see [Sol3, Lemma 3.1.7] or [Sol2, Lemma 3.4]. Since every $\mathcal{G}$-orbit in $\Xi$ contains an element in positive position, we may assume that $(Q, \delta, t)$ is positive. Then $\left(Q, \delta, t|t|^{r}\right)$ is positive for $r \geq-1$ and for $r>-1$ its stabilizer in $\mathcal{G}$ does not depend on $r$.

Now the statement for $\pi^{\Gamma}(Q, \delta, t)$ is an instance of [Sol3, Proposition 3.4.1]. Together with (37) and Theorem 1.10,c this implies the statement for $\pi(Q, \sigma, t)$.

(b) By [Sol3, Proposition 3.1.4.a] the representation

$$
\operatorname{ind}_{\mathcal{H}^{Q}}^{\mathcal{H}^{P(\xi)} \rtimes \Gamma(P(\xi), P(\xi))}\left(\delta \otimes t|t|^{r}\right)=\bigoplus_{i} \operatorname{ind}_{\mathcal{H}^{Q} \rtimes \Gamma_{Q}}^{\mathcal{H}^{P(\xi)} \rtimes \Gamma(P(\xi), P(\xi))}\left(\sigma_{i} \otimes t|t|^{r}\right)
$$

is completely reducible. Therefore it suffices to consider all irreducible direct summands of (39) separately. This brings us to representations of the form

$$
\operatorname{ind}_{\mathcal{H}^{P(\xi)} \rtimes \Gamma(P(\xi), P(\xi))}^{\mathcal{H} \rtimes \Gamma}\left(\delta^{\prime} \otimes t^{\prime}|t|^{r}\right) .
$$

Then $\left(P(\xi), \delta^{\prime}, t^{\prime}|t|^{r}\right)$ is a datum for the Langlands classification for extended affine Hecke algebras [Sol3, Corollary 2.2.5]. This result says that such a representation has a unique irreducible quotient, so (40) is irreducible as soon as it is completely reducible. In that case its Langlands constituent obviously is the whole of (40). That implies the claim about the constituents of $\pi\left(Q, \sigma, t|t|^{r}\right)$ when that representation is completely reducible.

Next we show that (40) is irreducible for almost all $r \in \mathbb{R}_{>-1}$. From Sol3, Lemma 2.2.6.a] we know that the space of $\mathcal{H} \rtimes \Gamma$-endomorphisms of (40) is just $\mathbb{C I d}$.

For all $z \in \mathbb{C}$ the isotropy group $\mathcal{G}_{\left(P(\xi), \delta^{\prime}, t^{\prime}\right)}$ also fixes $\left(P(\xi), \delta^{\prime}, t^{\prime}|t|^{z}\right)$. Since $\mathcal{G}_{P(\xi), \delta^{\prime}}$ acts on $T^{Q}$ by group automorphisms and translations, for almost all $z \in \mathbb{C}$ $\mathcal{G}_{\left(P(\xi), \delta^{\prime}, t^{\prime}|t|^{z}\right)}$ equals $\mathcal{G}_{\left(P(\xi), \delta^{\prime}, t^{\prime}\right)}$. In particular this happens for some $z \in-1+i \mathbb{R}$. Then $t^{\prime}|t|^{z} \in T_{\mathrm{un}}^{P(\xi)}$ and by [Sol3, Corollary 3.1.3] the representation $\pi^{\Gamma}\left(P(\xi), \delta^{\prime}, t^{\prime}|t|^{z}\right)$ is unitary (and in particular completely reducible). By [Sol3, Theorem 3.3.1.b]

$$
\operatorname{End}_{\mathcal{H} \rtimes \Gamma}\left(\operatorname{ind}_{\mathcal{H}^{P(\xi)} \rtimes \Gamma(P(\xi), P(\xi))}^{\mathcal{H} \rtimes \Gamma}\left(\delta^{\prime} \otimes t^{\prime}|t|^{z}\right)\right)
$$

is spanned by intertwining operators coming from $\mathcal{G}_{P(\xi), \delta^{\prime}, t^{\prime}}$, just like for (40). Therefore (41) consists only of $\mathbb{C I d}$, which implies that the representation is irreducible for that $z \in-1+i \mathbb{R}$. In the above algebraic family of finite dimensional representations parametrized by $z \in \mathbb{C}$, irreducibility is an open condition: slightly varying $z$ cannot destroy irreducibility. Hence the locus of $z$ 's where the representation is reducibile is a Zariski-closed subset of $\mathbb{C}$, that is, it is finite. In particular (40) is irreducible for all but finitely many $r \in \mathbb{R}_{>-1}$. 


\section{REDUCTIVE $p$-ADIC GROUPS}

Let $F$ be a non-archimedean local field and let $G=\mathcal{G}(F)$ be a connected reductive algebraic group over $F$. We endow $G$ with the topology coming from the metric on $F$ and we fix a Haar measure on $G$. Let $\mathcal{H}(G)$ be the Hecke algebra of $G$, the convolution algebra of locally constant compactly supported functions $G \rightarrow \mathbb{C}$. The product on $\mathcal{H}(G)$ is convolution (with respect to the Haar measure). Let $\mathcal{S}(G)$ be the Harish-Chandra-Schwartz algebra of $G$, as defined in [HC] and [Wal, §III.6]. By definition, a smooth $G$-representation (by default on a complex vector space) is tempered if and only if it extends continuously to a module for $\mathcal{S}(G)$. Let $C_{r}^{*}(G)$ be the reduced $C^{*}$-algebra of $G$, the completion of $\mathcal{H}(G)$ in the algebra of bounded linear operators on the Hilbert space $L^{2}(G)$. By [Vig, Theorem 29] there are dense inclusions

$$
\mathcal{H}(G) \subset \mathcal{S}(G) \subset C_{r}^{*}(G) .
$$

The *-operation and the trace on these algebras are

$$
f^{*}(g)=\overline{f\left(g^{-1}\right)} \text { and } \tau(f)=f\left(1_{G}\right) .
$$

Fix a minimal parabolic $F$-subgroup $P_{0}=M_{0} U_{0}$ and let $W\left(G, M_{0}\right)$ be the Weyl group of $G$ with respect to the maximal $F$-split torus $A_{0}$ in the centre of $M_{0}$. Write $\mathfrak{a}_{0}=X^{*}\left(A_{M_{0}}\right) \otimes_{\mathbb{Z}} \mathbb{R}$ and endow this vector space with a $W\left(G, M_{0}\right)$-invariant inner product.

Every Levi $F$-subgroup of $G$ is conjugate to a standard Levi subgroup, that is, one that contains $M_{0}$. Let $M$ be such a standard Levi subgroup of $G$, and let $A_{M}$ be the maximal $F$-split torus in $Z(M)$. There is a canonical decomposition

$$
\mathfrak{a}_{0}=\mathfrak{a}_{M} \oplus \mathfrak{a}^{M},
$$

where $\mathfrak{a}_{M}=X^{*}\left(A_{M}\right) \otimes_{\mathbb{Z}} \mathbb{R}$ and $\mathfrak{a}^{M}=\left\{\chi \in X^{*}\left(A_{0}\right):\left.\chi\right|_{A_{M}}=1\right\} \otimes_{\mathbb{Z}} \mathbb{R}$. Let $R(G, M)$ be the set of roots of $G$ with respect to $A_{M}$. For a parabolic subgroup $P=M U$ of $G$ we let $R(P, M)$ be the set of roots of $(G, M)$ that appear in (the Lie algebra of) $P$.

When $M_{1} \subset M$ is another standard Levi subgroup of $G$, we write $\mathfrak{a}_{M_{1}}^{M}=\mathfrak{a}_{M_{1}} \cap \mathfrak{a}^{M}$. Every parabolic subgroup $P_{1}=M_{1} U_{1}$ of $M$ determines an obtuse cone in $\mathfrak{a}_{M_{1}}^{M}$ :

$$
{ }^{+} \mathfrak{a}_{P_{1}}^{M}=\left\{\left.\sum_{\alpha \in R\left(P_{1}, M_{1}\right)} c_{\alpha} \alpha\right|_{A_{M_{1}}}: c_{\alpha}>0 \forall \alpha\right\} .
$$

Here we would obtain the same cone if we used only the simple roots for $\left(P_{1}, M_{1}\right)$. The closure of ${ }^{+} \mathfrak{a}_{P_{1}}^{M}$ in $\mathfrak{a}_{M_{1}}^{M}$ is

$$
\overline{{ }^{\mathfrak{a}_{P_{1}}^{M}}}=\left\{\left.\sum_{\alpha \in R\left(P_{1}, M_{1}\right)} c_{\alpha} \alpha\right|_{A_{M_{1}}}: c_{\alpha} \geq 0 \forall \alpha\right\} .
$$

It is easy to see that the normalized Jacquet restriction functor $J_{P}^{G}: \operatorname{Rep}(G) \rightarrow$ $\operatorname{Rep}(M)$ does not preserve temperedness. Fortunately, the normalized parabolic induction functor $I_{P}^{G}: \operatorname{Rep}(M) \rightarrow \operatorname{Rep}(G)$ does, and also respects non-temperedness:

Proposition 2.1. Let $\pi \in \operatorname{Rep}(M)$ be of finite length. Then $I_{P}^{G}(\pi)$ is tempered if and only if $\pi$ is tempered.

Proof. The if part is well-known, see [Wal, Lemme III.2.3] or [Ren, Lemme VII.2.2].

By conjugating $P, M$ and $\pi$, we can achieve that $P \supset P_{0}$ and $M \supset M_{0}$. Recall from [Wal, Proposition III.2.2] that $\pi$ is tempered if and only if, for every parabolic 
subgroup $P_{1}=M_{1} U_{1}$ of $M$ with $M_{1} \supset M_{0}$ and every $A_{M_{1}}$-weight $\chi$ of $J_{P_{1}}^{M}(\pi)$ :

$$
\log |\chi| \in \overline{{ }^{+} \mathfrak{a}_{P_{1}}^{M}} \text {. }
$$

Moreover, it is equivalent to impose this condition for all $P_{1}$ such that $P_{1}=M$ or $P_{1}$ is a standard maximal parabolic subgroup of $M$.

To show that $I_{P}^{G}$ preserves non-temperedness, it suffices to consider the case that $P$ is a standard maximal parabolic subgroup of $G$. Namely, there exists a chain of parabolic subgroups

$$
P \subset P_{1}=M_{1} U_{1} \subset \cdots \subset P_{n}=M_{n} U_{n} \subset G=M_{n+1}
$$

such that every $P_{i-1} \cap M_{i}$ is a maximal parabolic subgroup of $M_{i}$. If we can prove that each $I_{P_{i-1} \cap M_{i}}^{M_{i}}$ preserves non-temperedness, the transitivity of parabolic induction [Ren, Lemme VI.1.4] implies that $I_{P}^{G}$ does so as well.

Since $I_{P}^{G}$ is an exact functor [Ren, Théorème VI.1.1], we may furthermore assume that $\pi$ is irreducible. So, we suppose that $\pi$ is irreducible and not tempered, and (contrary to what we want to prove) that $I_{P}^{G}(\pi)$ is tempered. Let us consider the $Z(G)$-character of $\pi$. It is also the $Z(G)$-character of $I_{P}^{G}(\pi)$. Since $I_{P}^{G}(\pi)$ is tempered, its central character is unitary [Ren, Corollaire VII.2.6].

We claim that the $A_{M}$-character $\zeta$ of $\pi$ must also be unitary.

Suppose it is not, and consider its absolute value $|\zeta| \in X_{\mathrm{nr}}(M) \backslash\{1\}$. Let $\alpha \in$ $R\left(G, M_{0}\right)$ be the unique simple root of $(G, M)$ and let $s_{\alpha} \in W\left(G, M_{0}\right)$ be the associated reflection. The length of $s_{\alpha}$ in $W\left(G, M_{0}\right)$ is one, so it is a minimal length representative for a double coset in $W\left(M, M_{0}\right) \backslash W\left(G, M_{0}\right) \backslash W\left(M, M_{0}\right)$. By Bernstein's geometric lemma [Ren, Théorème VI.5.1] both $\zeta$ and $s_{\alpha} \zeta$ occur as $A_{M^{-}}$ weights of $J_{P}^{G}\left(I_{P}^{G}(\pi)\right)$. Since $M$ is a maximal Levi subgroup of $G$ and $|\zeta|_{A_{G}}=$ $|\zeta|_{Z(G)}=1$, both $\log |\zeta|$ and $\log \left|s_{\alpha} \zeta\right|$ lie in the one-dimensional vector space $\mathfrak{a}_{M}^{G}$. The reflection $s_{\alpha}$ acts as -1 on $\mathfrak{a}_{M}^{G}$, so $\left|s_{\alpha} \zeta\right|=|\zeta|^{-1}$. As $|\zeta| \neq 1$, it is not possible that

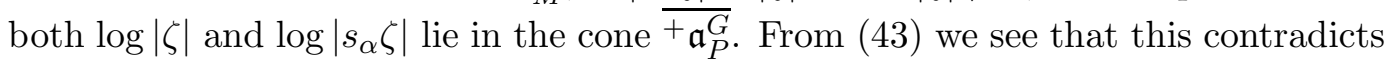
the temperedness of $I_{P}^{G}(\pi)$. Consequently $|\zeta|$ must be 1 , and $\zeta$ must be unitary.

Now we invoke the non-temperedness of $\pi$. By [Wal, Proposition III.2.2.iii] there exists a standard parabolic subgroup $P^{\prime}=M^{\prime} U^{\prime}$ of $G$ such that:

- $M^{\prime}=M$ or $M^{\prime}$ is a maximal Levi subgroup of $M$;

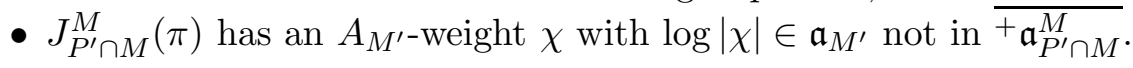

When $M^{\prime}=M$, then $\chi=\zeta$ and the above claim says that $|\chi|=1$. That would be in contradiction with the second bullet.

Hence $M^{\prime} \subsetneq M$. As $\left.\chi\right|_{A_{M}}=\zeta_{A_{M}}$ is unitary, $\chi$ is of the form $c_{\beta} \beta$, where $\beta \in$ $R\left(M, M_{0}\right)$ is the unique simple root for $\left(M, M^{\prime}\right)$. Then the second bullet says that $c_{\beta}<0$. By Bernstein's geometric lemma [Ren, Théorème VI.5.1] $\chi$ also a $A_{M^{\prime} \text {-weight }}$

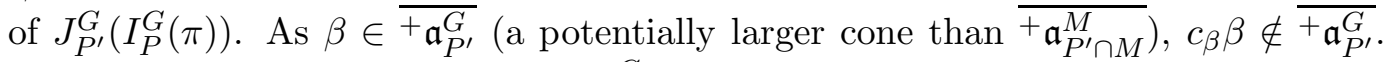
Together with (43) this shows that $I_{P}^{G}(\pi)$ cannot be tempered.

Let $L=\mathcal{L}(F)$ be a Levi subgroup $G$ and let $\left(\sigma, V_{\sigma}\right) \in \operatorname{Irr}(L)$ be an irreducible tempered supercuspidal $L$-representation. Let $X_{\mathrm{nr}}(L)$ be the group of unramified characters $L \rightarrow \mathbb{C}^{\times}$and let $X_{\text {unr }}(L)$ be the subgroup of unitary unramified characters. Recall that the inertial equivalence class of the pair $(L, \sigma)$ consists of all pairs of the form

$$
\left(g L g^{-1},(g \cdot \sigma) \otimes \chi\right) \text {, where } g \in G \text { and } \chi \in X_{\mathrm{nr}}\left(g L g^{-1}\right) .
$$


We write $\mathfrak{s}=[L, \sigma]_{G}$ and call this an inertial equivalence class for $G$. It gives rise to a subset $\operatorname{Irr}(G)^{\mathfrak{s}} \subset \operatorname{Irr}(G)$, namely all those irreducible smooth $G$-representations whose supercuspidal support lies in $\mathfrak{s}$. This in turn is used to define a subcategory $\operatorname{Rep}(G)^{\mathfrak{s}}$ of $\operatorname{Rep}(G)$, namely those smooth $G$-representations all whose irreducible constituents lie in $\operatorname{Irr}(G)^{\mathfrak{s}}$. The Bernstein blocks $\operatorname{Rep}(G)^{\mathfrak{s}}$ have better finiteness properties than $\operatorname{Rep}(G)$ :

Theorem 2.2. [Kaz, $\S 1.6]$

(a) Let $M \subset G$ be a Levi subgroup containing $L$. There exist tempered $\pi_{M, i} \in$ $\operatorname{Irr}(M)^{[L, \sigma]_{M}}\left(i=1, \ldots, \kappa_{M}\right)$, such that $\left\{\pi_{M, i} \otimes \chi_{M}: i=1, \ldots, \kappa_{M}, \chi_{M} \in\right.$ $\left.X_{\mathrm{nr}}(M)\right\}$ is the collection of irreducible representations in $\operatorname{Rep}(M)^{[L, \sigma]_{M}}$ that are not isomorphic to the normalized parabolic induction of representation of a proper Levi subgroup of $M$.

(b) Let $M$ run through a set of representatives for the conjugacy classes of Levi subgroups of $G$ containing $L$. Then the set

$$
\bigcup_{M}\left\{I_{P}^{G}\left(\pi_{M, i} \otimes \chi_{M}\right): i=1, \ldots, \kappa_{M}, \chi_{M} \in X_{\mathrm{nr}}(M)\right\}
$$

spans the Grothendieck group of the category of finite length representations in $\operatorname{Rep}(G)^{\mathfrak{s}}$.

Let $\mathfrak{B}(G)$ be the set of all inertial equivalence classes $\mathfrak{s}$ for $G$. By BeDe, Corollaire 3.9] it is countably infinite (unless $G=1$ ). The Bernstein decomposition BeDe, Theorem 2.10] says that

$$
\begin{aligned}
& \operatorname{Rep}(G)=\prod_{\mathfrak{s} \in \mathfrak{B}(G)} \operatorname{Rep}(G)^{\mathfrak{s}}, \\
& \mathcal{H}(G)=\bigoplus_{\mathfrak{s} \in \mathfrak{B}(G)} \mathcal{H}(G)^{\mathfrak{s}},
\end{aligned}
$$

where $\mathcal{H}(G)^{\mathfrak{s}}$ is the two-sided ideal of $\mathcal{H}(G)$ for which $\operatorname{Mod}\left(\mathcal{H}(G)^{\mathfrak{s}}\right)$ is naturally equivalent with $\operatorname{Rep}(G)^{\mathfrak{s}}$.

Let $\mathcal{S}(G)^{\mathfrak{s}}$ (resp. $\left.C_{r}^{*}(G)^{\mathfrak{s}}\right)$ be the two-sided ideal of $\mathcal{S}(G)$ (resp. $C_{r}^{*}(G)$ ) generated by $\mathcal{H}(G)^{\mathfrak{s}}$. Upon completion, (44) yields further Bernstein decompositions

$$
\begin{aligned}
& \mathcal{S}(G)=\bigoplus_{\mathfrak{s} \in \mathfrak{B}(G)} \mathcal{S}(G)^{\mathfrak{s}}, \\
& C_{r}^{*}(G)=\bigoplus_{\mathfrak{s} \in \mathfrak{B}(G)} C_{r}^{*}(G)^{\mathfrak{s}} .
\end{aligned}
$$

The latter must be interpreted as a direct sum in the Banach algebra sense: it is the completion of the algebraic direct sum with respect to the operator norm of $C_{r}^{*}(G)$.

For a compact open subgroup $K$ of $G$ we let $\langle K\rangle$ be the corresponding idempotent of $\mathcal{H}(G)$. Then

$$
\mathcal{H}(G, K):=\langle K\rangle \mathcal{H}(G)\langle K\rangle
$$

is the subalgebra of $K$-biinvariant functions in $\mathcal{H}(G)$. We define $\mathcal{S}(G, K)$ and $C_{r}^{*}(G, K)$ analogously. For every compact open subgroup $K$ of $G, \mathcal{S}(G, K)$ is a Fréchet algebra $\mathrm{Vig}$, Theorem 29]. The Schwartz algebra $\mathcal{S}(G)$ is their union (over all possible $K)$, so it is an inductive limit of Fréchet algebras.

We will focus on one Bernstein block $\operatorname{Rep}(G)^{\mathfrak{s}}$ of $\operatorname{Rep}(G)$. By BeDe, Corollaire 3.9] there exists a compact open subgroup $K_{\mathfrak{s}}$ of $G$ such that every representation in $\operatorname{Rep}(G)^{\mathfrak{s}}$ is generated by its $K_{\mathfrak{s}}$-fixed vectors. This leads to Morita equivalences

$$
\begin{array}{ccccc}
\mathcal{H}(G)^{\mathfrak{s}} & \sim_{M} & \mathcal{H}\left(G, K_{\mathfrak{s}}\right)^{\mathfrak{s}} & := & \mathcal{H}(G)^{\mathfrak{s}} \cap \mathcal{H}\left(G, K_{\mathfrak{s}}\right) \\
\mathcal{S}(G)^{\mathfrak{s}} & \sim_{M} & \mathcal{S}\left(G, K_{\mathfrak{s}}\right)^{\mathfrak{s}} & := & \mathcal{S}(G)^{\mathfrak{s}} \cap \mathcal{S}\left(G, K_{\mathfrak{s}}\right) \\
C_{r}^{*}(G)^{\mathfrak{s}} & \sim_{M} & C_{r}^{*}\left(G, K_{\mathfrak{s}}\right)^{\mathfrak{s}} & := & C_{r}^{*}(G)^{\mathfrak{s}} \cap C_{r}^{*}\left(G, K_{\mathfrak{s}}\right) .
\end{array}
$$




\subsection{The Plancherel isomorphism.}

We will describe the structure of $\mathcal{S}(G)^{\mathfrak{s}}$ and $\mathcal{S}\left(G, K_{\mathfrak{s}}\right)^{\mathfrak{s}}$ in more detail. Let $[L, \sigma]_{L}=T_{\mathfrak{s}} \subset \operatorname{Irr}(L)$ be the set of $L$-representations of the form $\sigma \otimes \chi$ with $\chi \in X_{\mathrm{nr}}(L)$. Thus there is a finite covering of complex varieties

$$
X_{\mathrm{nr}}(L) \rightarrow T_{\mathfrak{s}}: \chi \mapsto \sigma \otimes \chi .
$$

Let $T_{\mathfrak{s}, \text { un }}$ be the subset of unitary representations in $T_{\mathfrak{s}}$, it is covered by $X_{\text {unr }}(L)$ via (48). We write

$$
X_{\mathrm{nr}}(L, \sigma)=\left\{\chi \in X_{\mathrm{nr}}(L): \sigma \otimes \chi \cong \sigma\right\} .
$$

This is a finite subgroup of $X_{\mathrm{unr}}(L)$. The map (48) induces an isomorphism of algebraic varieties $X_{\mathrm{nr}}(L) / X_{\mathrm{nr}}(L, \sigma) \rightarrow T_{\mathfrak{s}}$.

The group $W(G, L)=N_{G}(L) / L$ acts on $\operatorname{Irr}(L)$ by

$$
(g L \cdot \pi)(l)=\pi\left(g l g^{-1}\right) .
$$

(The representation $g L \cdot \pi$ is only determined up to isomorphism.) This action stabilizes $X_{\mathrm{nr}}(L)$, the unitary representations in $\operatorname{Irr}(L)$ and the supercuspidal $L$ representations. Let $W_{\mathfrak{s}}$ be the stabilizer of $T_{\mathfrak{s}}$ in $N_{G}(L) / L$. This group will play the same role as $W \Gamma$ did in Section 1. The theory of the Bernstein centre BeDe, Théor'eme 2.13] says that the centre of $\mathcal{H}\left(G, K_{\mathfrak{s}}\right)^{\mathfrak{s}}$ is naturally isomorphic with $\mathcal{O}\left(T_{\mathfrak{s}}\right)^{W_{\mathfrak{s}}}=\mathcal{O}\left(T_{\mathfrak{s}} / W_{\mathfrak{s}}\right)$.

It will be convenient to lift everything from $T_{\mathfrak{s}}$ to $X_{\mathrm{nr}}(L)$. However, $W_{\mathfrak{s}}$ does not act naturally on $X_{\mathrm{nr}}(L)$. To overcome this and similar issues, we need the following lemma.

Lemma 2.3. Let $p: T^{\prime} \rightarrow T$ be a surjection between complex tori, with finite kernel $K=\operatorname{ker} p$. Let $\Gamma$ be a finite group acting on $T$ by automorphisms of algebraic varieties (so $\Gamma$ need not fix $1 \in T$ ). Then there exists a canonical short exact sequence

$$
1 \rightarrow K \rightarrow \Gamma^{\prime} \rightarrow \Gamma \rightarrow 1
$$

and a canonical action of $\Gamma^{\prime}$ on $T^{\prime}$ which extends the multiplication action of $K$ on $T^{\prime}$ and lifts the action of $\Gamma$ on $T$.

Proof. Let $X$ be the character lattice of $T$. Then $\mathcal{O}(T) \cong \mathbb{C}[X]$ and $\Gamma$ acts on $\mathcal{O}(T)$ by $(\gamma \cdot f)(t)=f\left(\gamma^{-1} t\right)$. Since

$$
\mathcal{O}(T)^{\times}=\left\{z \theta_{x}: z \in \mathbb{C}^{\times}, x \in X\right\} \cong \mathbb{C}^{\times} \times X,
$$

$\Gamma$ also acts naturally on $X \cong \mathcal{O}(T)^{\times} / \mathbb{C}^{\times}$. Let us denote this action by $l_{\gamma}: X \rightarrow X$. Notice that it defines an action of $\Gamma$ on $T=\operatorname{Hom}_{\mathbb{Z}}\left(X, \mathbb{C}^{\times}\right)$by algebraic group automorphisms. The given action on $\mathcal{O}(T)$ can now be written as

$$
\gamma\left(z \theta_{x}\right)=z z_{\gamma}^{-1}\left(l_{\gamma}(x)\right) \theta_{l_{\gamma}(x)},
$$

for a unique $z_{\gamma} \in T$. Consequently the original action of $\Gamma$ on $T$ can be expressed as

$$
\gamma(t)=z_{\gamma} l_{\gamma}(t)
$$

The character lattice $X^{\prime}$ of $T^{\prime}$ contains $X$ with finite index $|K|$, so $l_{\gamma}$ induces a canonical linear action of $\Gamma$ on $X^{\prime}$, also denoted $l_{\gamma}$. For every $\gamma \in \Gamma$ we choose a $z_{\gamma}^{\prime} \in p^{-1}\left(z_{\gamma}\right)$, and we define

$$
\phi_{\gamma}: T^{\prime} \rightarrow T^{\prime}, \quad \phi_{\gamma}\left(t^{\prime}\right)=z_{\gamma}^{\prime} l_{\gamma}\left(t^{\prime}\right) .
$$


Clearly $\phi_{\gamma}$ is a lift of (50), so for every $\gamma, \gamma^{\prime} \in \Gamma$ there exists a unique $z_{\gamma, \gamma^{\prime}}^{\prime} \in K$ with

$$
\phi_{\gamma} \circ \phi_{\gamma^{\prime}} \circ \phi_{\gamma \gamma^{\prime}}^{-1}\left(t^{\prime}\right)=z_{\gamma, \gamma^{\prime}}^{\prime} t^{\prime} \quad \forall t^{\prime} \in T^{\prime} .
$$

Let $\Gamma^{\prime}$ be the subgroup of $\operatorname{Aut}\left(T^{\prime}\right)$ generated by the $\phi_{\gamma}(\gamma \in \Gamma)$ and $K$. Then (51) gives a canonical isomorphism $\Gamma^{\prime} / K \cong \Gamma$.

The only unnatural steps in the above argument are the choices of the $z_{\gamma}^{\prime}$. Different choices would lead to different $z_{\gamma, \gamma^{\prime}}^{\prime}$ in (51), but to the same group $\Gamma^{\prime}$. Hence $\Gamma^{\prime}$ is canonically determined by the data $T, T^{\prime}$ and $\Gamma$.

Next we recall the Plancherel isomorphism for $\mathcal{S}(G)^{\mathfrak{s}}$, as discovered by HarishChandra and worked out by Waldspurger. As induction data for $G$ we take quadruples $(P, M, \omega, \chi)$, where

- $P$ is a parabolic subgroup of $G$ with a Levi factor $M$;

- $\left(\omega, V_{\omega}\right) \in \operatorname{Irr}_{L^{2}}(M)$, the set of (isomorphism classes of) irreducible smooth square-integrable modulo centre representations of $M$;

- $\chi \in X_{\mathrm{nr}}(M)$.

To such a datum we associate the smooth $G$-representation $I_{P}^{G}(\omega \otimes \chi)$, where $I_{P}^{G}$ denotes normalized parabolic induction. When $\chi$ is unitary, the $M$-invariant inner product on $\left(\omega \otimes \chi, V_{\omega}\right)$ induces a $G$-invariant inner product on $I_{P}^{G}\left(V_{\omega}\right)$, so $I_{P}^{G}(\omega \otimes \chi)$ is pre-unitary [Cas, Proposition 3.1.4]. However, $I_{P}^{G}\left(V_{\omega}\right)$ is only complete with respect to the associated metric if $\operatorname{dim}\left(I_{P}^{G}\left(V_{\omega}\right)\right)$ is finite.

Let $\left(\check{\omega}, \check{V}_{\omega}\right)$ be the smooth contragredient of $\omega$ and put

$$
\mathfrak{L}(\omega, P)=I_{P \times P}^{G \times G}(\omega \otimes \check{\omega})=I_{P}^{G}(\omega) \otimes I_{P}^{G}(\check{\omega}) .
$$

Since $I_{P}^{G}(\check{\omega})$ can be identified with the smooth contragredient of $I_{P}^{G}(\omega)$ [Cas, Proposition 3.1.2], $\mathfrak{L}(\omega, P)$ can be regarded as the algebra of finite rank linear operators on $I_{P}^{G}\left(V_{\omega}\right)$. Notice that for every $\chi \in X_{\mathrm{nr}}(M)$ we can identify $\mathfrak{L}(\omega \otimes \chi, P)$ with $\mathfrak{L}(\omega, P)$ as algebras. The inner product on $I_{P}^{G}\left(V_{\omega}\right)$ induces a ${ }^{*}$-operation on this algebra. That makes $\mathcal{O}\left(X_{\mathrm{nr}}(M)\right) \otimes \mathfrak{L}(\omega, P)$ to a ${ }^{*}$-algebra with

$$
f^{*}(\chi)=f(\check{\chi})^{*} \text {. }
$$

There is a natural *-homomorphism

$$
\begin{gathered}
\mathcal{H}(G) \quad \rightarrow \mathcal{O}\left(X_{\mathrm{nr}}(M)\right) \otimes \mathfrak{L}(\omega, P), \\
f \\
\mapsto\left(\chi \mapsto I_{P}^{G}(\omega \otimes \chi)(f)\right) .
\end{gathered}
$$

We put $T_{\omega}=\left\{\omega \otimes \chi: \chi \in X_{\mathrm{nr}}(M)\right\}$ and we record the covering map

$$
X_{\mathrm{nr}}(M) \rightarrow T_{\omega}: \chi \mapsto \omega \otimes \chi .
$$

The group

$$
X_{\mathrm{nr}}(M, \omega)=\left\{\chi \in X_{\mathrm{nr}}(M): \omega \otimes \chi \cong \omega\right\}
$$

is finite, because all its elements must be trivial on $Z(M)$. All the fibres of (53) are isomorphic to $X_{\mathrm{nr}}(M, \omega)$.

For every $k \in X_{\mathrm{nr}}(M, \omega)$ there exists a unitary $M$-intertwiner $\omega \rightarrow \omega \otimes k$, unique up to scalars. The same map $V_{\omega} \rightarrow V_{\omega}$ also intertwines $\omega \otimes \chi$ with $\omega \otimes \chi k$, for any $\chi \in X_{\mathrm{nr}}(M)$. Applying $I_{P}^{G}$, we get a family a $G$-intertwiners

$$
\pi(k, \omega, \chi): I_{P}^{G}(\omega \otimes \chi) \rightarrow I_{P}^{G}(\omega \otimes \chi k),
$$


independent of $\chi$ and unitary when $\chi \in X_{\text {unr }}(M)$. Let

$$
\left.\check{\pi}(k, \omega, \chi): I_{P}^{G}(\check{\omega} \otimes \check{\chi})\right) \rightarrow I_{P}^{G}(\check{\omega} \otimes \check{\chi} k)
$$

be the inverse transpose $\pi(k, \omega, \chi)$. Since $\pi(k, \omega, \chi)$ is unique up to scalars,

$$
I(k, \omega, \chi):=\pi(k, \omega, \chi) \otimes \check{\pi}(k, \omega, \chi) \in \operatorname{Hom}_{G \times G}(\mathfrak{L}(\omega \otimes \chi, P), \mathfrak{L}(\omega \otimes \chi k, P))
$$

is canonical. Moreover it is unitary for $\chi \in X_{\mathrm{unr}}(M)$ and independent of $\chi$ as map between vector spaces.

Let $W\left(T_{\omega}\right)$ be the stabilizer of $T_{\omega}$ in $W(G, M)=N_{G}(M) / M$, with respect to the action on $\operatorname{Irr}(M)$ as in (49). Then $W\left(T_{\omega}\right)$ acts naturally on $T_{\omega}$. From Lemma 2.3 we get a group extension

$$
1 \rightarrow X_{\mathrm{nr}}(M, \omega) \rightarrow W^{\prime}\left(T_{\omega}\right) \rightarrow W\left(T_{\omega}\right) \rightarrow 1
$$

and an action of $W^{\prime}\left(T_{\omega}\right)$ on $X_{\mathrm{nr}}(M)$ compatible with the covering (53). In [Wal] the representations $\omega \otimes \chi$ and $\omega \otimes \chi k$ are often not distinguished. The introduction of $W^{\prime}\left(T_{\omega}\right)$ and of the $I(k, \omega, \chi)$ allows us to compare $I_{P}^{G}(\omega \otimes \chi)$ and $I_{P}^{G}(\omega \otimes \chi k)$ in a systematic way. From [Wal, $\S V I .1]$ one can see that actually our setup is just another way to keep track of all the ingredients of [Wal].

The following results are proven in [Wal, Paragraphe V]. For $w^{\prime} \in W^{\prime}\left(T_{\omega}\right)$ there exist unitary $G$-intertwining operators

$$
\pi\left(w^{\prime}, \omega, \chi\right): I_{P}^{G}(\omega \otimes \chi) \rightarrow I_{P}^{G}\left(\omega \otimes w^{\prime}(\chi)\right) \quad \chi \in X_{\mathrm{unr}}(M),
$$

unique up to scalars. These give canonical unitary intertwiners

$$
I\left(w^{\prime}, \omega, \chi\right)=\pi\left(w^{\prime}, \omega, \chi\right) \otimes \check{\pi}\left(w^{\prime}, \omega, \chi\right) \in \operatorname{Hom}_{G \times G}\left(\mathfrak{L}(\omega \otimes \chi, P), \mathfrak{L}\left(\omega \otimes w^{\prime}(\chi), P\right)\right)
$$

with the following properties [Wal, Lemme V.3.1]:

- as functions of $\chi, \pi\left(w^{\prime}, \omega, \chi\right)$ and $I\left(w^{\prime}, \omega, \chi\right)$ are continuous with respect to the Zariski topology on the real algebraic variety $X_{\text {unr }}(M)$;

- $I\left(w_{2}^{\prime}, \omega, w_{1}^{\prime}(\chi)\right) \circ I\left(w_{1}^{\prime}, \omega, \chi\right)=I\left(w_{2}^{\prime} w_{1}^{\prime}, \omega, \chi\right)$ for $w_{1}^{\prime}, w_{2}^{\prime} \in W^{\prime}\left(T_{\omega}\right)$.

The properties of the intertwining operators (57) imply that, for every $g \in G, \omega \in$ $\operatorname{Irr}_{L^{2}}(M), \quad \chi \in X_{\mathrm{nr}}(M)$ and every parabolic subgroup $P^{\prime} \subset G$ with Levi factor $g M g^{-1}$, the representations $I_{P}^{G}(\omega \otimes \chi)$ and $I_{P^{\prime}}^{G}(g \cdot \omega \otimes g \cdot \chi)$ have the same irreducible subquotients, counted with multiplicity [Sol1, Corollary 2.7].

We remark that $I\left(w^{\prime}, \omega, \chi\right)$ is called ${ }^{\circ} c_{P \mid P}\left(w^{\prime}, \omega \otimes \chi\right)$ in Wal]. The intertwining operators (58) give rise to an action of $W^{\prime}\left(T_{\omega}\right)$ on the algebra

$$
C^{\infty}\left(X_{\mathrm{unr}}(M)\right) \otimes \mathfrak{L}(\omega, P) \quad \text { by } \quad\left(w^{\prime} \cdot f\right)\left(w^{\prime} \chi\right)=I\left(w^{\prime}, \omega, \chi\right) f(\chi) .
$$

We fix a parabolic subgroup $P_{L}$ with Levi factor $L$, and we recall that $\mathfrak{s}=[L, \sigma]_{G}$. To study representations in the Bernstein block $\operatorname{Rep}(G)^{\mathfrak{s}}$, it suffices to consider induction data such that $P \supset P_{L}, M \supset L$ and the cuspidal support of $\omega$ lies in $[L, \sigma]_{M}$. Then $W\left(T_{\omega}\right)$ can be regarded as a subgroup of $W_{\mathfrak{s}}$.

Choose representatives for the $G$-association classes of parabolic subgroups $P$ containing $P_{L}$. Notice that every such $P$ has a unique Levi factor $M$ containing $L$. We also choose representatives $\omega$ for the action of $W_{\mathfrak{s}} \ltimes X_{\text {unr }}(M)$ on $\operatorname{Irr}_{L^{2}}(M) \cap$ $\operatorname{Irr}(M)^{\mathfrak{s}_{M}}$, where $\mathfrak{s}_{M}=[L, \sigma]_{M}$. We denote the resulting set of representative triples by $(P, M, \omega) / \sim$. Harish-Chandra established the following Plancherel isomorphism, see [ScZi, Theorem 8.9] for an alternative proof. 
Theorem 2.4. [Wal, Théorème VII.2.5]

The maps (52) induces isomorphisms of topological *algebras

$$
\begin{array}{ll}
\mathcal{S}(G)^{\mathfrak{s}} & \rightarrow \bigoplus_{(P, M, \omega) / \sim}\left(C^{\infty}\left(X_{\mathrm{unr}}(M)\right) \otimes \mathfrak{L}(\omega, P)\right)^{W^{\prime}\left(T_{\omega}\right)}, \\
\mathcal{S}\left(G, K_{\mathfrak{s}}\right)^{\mathfrak{s}} & \rightarrow \bigoplus_{(P, M, \omega) / \sim}\left(C^{\infty}\left(X_{\mathrm{unr}}(M)\right) \otimes \operatorname{End}_{\mathbb{C}}\left(I_{P}^{G}\left(V_{\omega}\right)^{K_{\mathfrak{s}}}\right)\right)^{W^{\prime}\left(T_{\omega}\right)} .
\end{array}
$$

Plymen $[\mathrm{Ply}]$ showed that Theorem 2.4 has a natural extension to $C^{*}$-algebras. Let $\mathfrak{H}(\omega \otimes \chi, P)$ be the Hilbert space completion of $I_{P}^{G}\left(V_{\omega \otimes \chi}\right)=I_{P}^{G}\left(V_{\omega}\right)$ and let $\mathfrak{K}(\omega \otimes \chi, P)$ be the $C^{*}$-algebra of compact operators on $\mathfrak{H}(\omega \otimes \chi, P)$.

Theorem 2.5. The maps (52) induces isomorphisms of $C^{*}$-algebras

$$
\begin{array}{ll}
C_{r}^{*}(G)^{\mathfrak{s}} & \rightarrow \bigoplus_{(P, M, \omega) / \sim} C\left(X_{\mathrm{unr}}(M) ; \mathfrak{K}(\omega, P)\right)^{W^{\prime}\left(T_{\omega}\right)}, \\
C_{r}^{*}\left(G, K_{\mathfrak{s}}\right)^{\mathfrak{s}} & \rightarrow \bigoplus_{(P, M, \omega) / \sim}\left(C\left(X_{\mathrm{unr}}(M)\right) \otimes \operatorname{End}_{\mathbb{C}}\left(I_{P}^{G}\left(V_{\omega}\right)^{K_{\mathfrak{s}}}\right)\right)^{W^{\prime}\left(T_{\omega}\right)} .
\end{array}
$$

Proof. First we note that we have intertwining operators associated to the group $W^{\prime}\left(T_{\omega}\right)$, instead of $W\left(T_{\omega}\right)$ in $[\mathrm{Ply}$, Wal. The reason for this is explained after (56). In view of Theorem 2.4, it only remains to prove that completing with respect to the operator norm of $C_{r}^{*}(G)$ boils down to replacing $C^{\infty}\left(X_{\mathrm{unr}}(M)\right) \otimes \mathfrak{L}(\omega, P)$ by $C\left(X_{\text {unr }}(M) ; \mathfrak{K}(\omega, P)\right)$. This is shown in Ply, Theorem 2.5].

\subsection{The space of irreducible representations.}

Like in Paragraph 1.2, we need more information about the space of all irreducible smooth $G$-representations (tempered or not). Suppose that $\pi \in \operatorname{Irr}(G)$ has supercuspidal support $\sigma \otimes \chi$, where $\sigma \in \operatorname{Irr}_{L^{2}}(M)$ and $\chi \in X_{\mathrm{nr}}(M)$. Then $\log |\chi| \in \mathfrak{a}_{M}$, and its image in $\mathfrak{a}_{0}$ is uniquely determined, up to $W\left(G, M_{0}\right)$, by $\pi$. In other words,

$$
c c(\pi):=W\left(G, M_{0}\right) \log |\chi|
$$

is an invariant of $\pi$. Since the norm on $\mathfrak{a}_{0}$ comes from a $W\left(G, M_{0}\right)$-invariant inner product, $\|c c(\pi)\|:=\|\log |\chi|\|$ is well-defined.

Theorem 2.6. (a) For every $\pi \in \operatorname{Irr}(G)$ there exists an induction datum

$(P, M, \omega, \chi)$, unique up to conjugation, such that $\pi$ is a constituent of $I_{P}^{G}(\omega \otimes \chi)$ and $\|c c(\omega)\|$ is maximal for this property. In this case we call $\pi$ a Langlands constituent of $I_{P}^{G}(\omega \otimes \chi)$.

(b) $\pi$ is tempered if and only if $\chi \in X_{\mathrm{unr}}(M)$.

(c) For any induction datum $(P, M, \omega, \chi)$, every constituent of $I_{P}^{G}(\omega \otimes \chi)$ is either a Langlands constituent or a constituent of some $I_{P^{\prime}}^{G}\left(\omega^{\prime} \otimes \chi^{\prime}\right)$ with $\left\|c c\left(\omega^{\prime}\right)\right\|>$ $\|c c(\omega)\|$.

(d) Suppose that $L$ is a standard Levi subgroup and that $\pi \in \operatorname{Irr}(G)^{\mathfrak{s}}$, where $\mathfrak{s}=$ $[L, \sigma]_{G}$. Then we can choose $(P, M, \omega, \chi)$ from part (a) such that $P \supset P_{0}, M \supset$ $L$ and $\omega \in \operatorname{Irr}(M)^{[L, \sigma]_{M}}$.

Proof. (a) See [Sol1, Theorem 2.15.b].

(b) This is a direct consequence of [Sol1, Proposition 2.14.b and Theorem 2.15.a].

(c) By [Sol1, Lemma 2.13] $(P, M, \omega, \chi)$ is equivalent to an induction datum $\xi^{+}$ in positive position. By [Sol1, Corollary 2.7] $I_{P}^{G}(\omega \otimes \chi)$ has the same irreducible subquotients, counted with multiplicity, as the parabolically induced representation associated to $\xi^{+}$. Therefore we may assume that $(P, M, \omega, \chi)$ is in positive position, that is, $P \supset P_{0}$ and $\log |\chi|$ lies in the closed positive cone in $\mathfrak{a}_{0}$ (determined by $P_{0}$ ). 
Then [Sol1, Theorem 2.15.a] says that the Langlands constituents of $I_{P}^{G}(\omega \otimes \chi)$ are precisely its irreducible quotients. Furthermore, by [Sol1, Proposition 2.15.a] $I_{P}^{G}(\omega \otimes$ $\chi)$ is a direct sum of representations of the form $I_{Q}^{G}(\tau \otimes|\chi|)$, where $(Q, \tau, \log |\chi|)$ is a datum for the Langlands classification of $\operatorname{Irr}(G)$. Suppose that $\pi^{\prime}$ is a constituent of $I_{Q}^{G}(\tau \otimes|\chi|)$, but not a quotient. By [Sol1, Lemma 2.11.a and Lemma 2.12], $\pi^{\prime}$ is the Langlands quotient of $I_{Q^{\prime}}^{G}\left(\tau^{\prime} \otimes \nu^{\prime}\right)$, for a Langlands datum $\left(Q^{\prime}, \tau^{\prime}, \log \nu^{\prime}\right)$ with $Q^{\prime} \supset Q$ and $\left\|c c\left(\tau^{\prime}\right)\right\|>\|c c(\tau)\|$. By [Sol1, Proposition 2.15.a] $\pi^{\prime}$ is a Langlands constituent of $I_{P^{\prime}}^{G}\left(\omega^{\prime} \otimes \chi^{\prime}\right)$, for some induction datum $\left(P^{\prime}, M^{\prime}, \omega^{\prime}, \chi^{\prime}\right)$ with

$$
\left\|c c\left(\omega^{\prime}\right)\right\|=\left\|c c\left(\tau^{\prime}\right)\right\|>\|c c(\tau)\|=\|c c(\omega)\| .
$$

(d) Let $P_{i}=M_{i} U_{i}$ be a standard parabolic subgroup and let $\overline{P_{i}}$ be the unique parabolic subgroup with Levi factor $M_{i}$ that is opposite to $P_{i}$. Let $J \frac{G}{P_{i}}: \operatorname{Rep}(G) \rightarrow$ $\operatorname{Rep}\left(M_{i}\right)$ be the normalized Jacquet restriction functor.

From [Ren, §VII.4.2] we recall how $\pi$ can be realized as a Langlands quotient. Namely, we take $\overline{P_{1}}$ such that $J \frac{G}{P_{1}}(\pi)$ contains a representation of the form $\tau \otimes \nu$, where $\left(P_{1}, \tau, \log \nu\right)$ is a Langlands datum. By [Wal, Proposition III.4.1] there exists a parabolic subgroup $P_{2}$ with $P_{0} \subset P_{2} \subset P_{1}$, and a $\omega^{\prime} \in \operatorname{Irr}_{L^{2}}\left(M_{2}\right)$, such that $\tau \otimes \nu$ is a direct summand of $I_{M_{1} \cap P_{2}}^{M_{1}}\left(\omega^{\prime} \otimes \nu\right)$. From the proof of part (c) we see that $\pi$ is a Langlands constituent of $I_{P_{2}}^{G}\left(\omega^{\prime} \otimes \nu\right)$. By the second adjointness theorem

$$
0 \neq \operatorname{Hom}_{G}\left(I_{P_{2}}^{G}\left(\omega^{\prime} \otimes \nu\right), \pi\right) \cong \operatorname{Hom}_{M_{2}}\left(\omega^{\prime} \otimes \nu, J \frac{G}{P_{2}}(\pi)\right) .
$$

The cuspidal support of $J \frac{G}{P_{2}}(\pi)$ equals that of $\pi$, so $\omega^{\prime} \otimes \nu$ also has cuspidal support in $[L, \sigma]_{G}$. More precisely, the cuspidal support of $\omega^{\prime} \otimes \nu$ is of the form $\left[L^{\prime}, \sigma^{\prime}\right]_{M_{2}}$, where $L^{\prime}$ is a standard Levi subgroup of $G$ conjugate to $L$. Since every Levi subgroup containing $L$ is $G$-conjugate to a standard Levi subgroup of $G$ containing $L$, we may replace $\left(P_{2}, M_{2}, \omega^{\prime}, \nu\right)$ by a $G$-conjugate $(P, M, \omega, \chi)$ with $M$ standard. Thus, we can arrange that the cuspidal support becomes $\left[L, \sigma^{\prime \prime}\right]_{M}$, for some cuspidal $\sigma^{\prime \prime} \in$ $\operatorname{Irr}(L)$. Then (60) is also valid for $I_{P}^{G}(\omega \otimes \chi)$, since $I_{P_{2}}^{G}\left(\omega^{\prime} \otimes \nu\right)$ is not affected by $G$ conjugation of $\left(P_{2}, M_{2}, \omega^{\prime}, \nu\right)$. Second adjointness tells us that $J \frac{G}{P}(\pi) \in \operatorname{Irr}(M)^{[L, \sigma]_{M}}$, so also $\omega \otimes \chi \in \operatorname{Irr}(M)^{[L, \sigma]_{M}}$. Finally, we may replace $P$ by a standard parabolic subgroup with Levi factor $M$, for this does not change the collection of constituents of $I_{P}^{G}(\omega \otimes \chi)$.

In [Sol1] Theorem 2.6 was used to study the geometry of $\operatorname{Irr}(G)$, and the relation with the subspace of tempered irreducible representations. For our purposes we need some aspects of that, and we need to know that for almost all induction data every constituent is a Langlands constituent.

Proposition 2.7. Let $(P, M, \omega, \chi)$ be an induction datum for $G$.

(a) For $r \in \mathbb{R}_{>-1}$, the number of inequivalent Langlands constituents of $I_{P}^{G}\left(\omega \otimes \chi|\chi|^{r}\right)$ does not depend on $r$.

(b) For all but finitely many $r \in \mathbb{R}_{>-1}, I_{P}^{G}\left(\omega \otimes \chi|\chi|^{r}\right)$ is completely reducible. Then all its irreducible subquotients are Langlands constituents.

Proof. (a) All the induction data under consideration have the same stabilizer in $W^{\prime}\left(T_{\omega}\right)$. As $W^{\prime}\left(T_{\omega}\right)$ is by construction the stabilizer of $T_{\omega}$ in the $\mathcal{W}$ from [Sol1, the statement is a special case of [Sol1, Lemma 2.16]. 
(b) As noted in the proof of Theorem 2.6. c, $I_{P}^{G}\left(\omega \otimes \chi|\chi|^{r}\right)$ is a direct sum of representations of the form $I_{Q}^{G}\left(\tau \otimes|\chi|^{r+1}\right)$, where $\left(Q=L U_{Q}, \tau, \log |\chi|^{r+1}\right)$ is a Langlands datum. Hence it suffices to show that $I_{Q}^{G}\left(\tau \otimes|\chi|^{r+1}\right)$ is irreducible for almost all $r \in \mathbb{R}_{>-1}$. The conditions of a Langlands datum say that $\tau \in \operatorname{Irr}(L)$ is tempered and that $\log |\chi|^{r+1} \in \mathfrak{a}_{L}$ is strictly positive with respect to the roots for $(Q, L)$. This implies that, for every $r \in \mathbb{R}_{>-1}$ and every root $\alpha$ for $(G, L),\left\langle\alpha, \log |\chi|^{r+1}\right\rangle \neq 0$. Now [Sau, Théorème 3.2] says that, for $r \in \mathbb{R}_{>-1}$ close enough to $-1, I_{Q}^{G}\left(\tau \otimes|\chi|^{r+1}\right)$ is irreducible. On the algebraic family of finite length representations $I_{Q}^{G}\left(\tau \otimes|\chi|^{r+1}\right)$ with $r \in \mathbb{R}$, irreducibility is an Zariski-open condition [Ren, Proposition VI.8.4]. Hence the locus of $r$ 's for which this representation is reducible is a finite set.

\section{Morita Equivalences}

In this section we will first formulate a long list of conditions for the objects we want to compare. Assuming these conditions, we will prove a comparison theorem. In the next sections we will check that these conditions are fulfilled in cases of interest.

\subsection{Conditions and first consequences.}

We keep the notations from the previous section.

Condition 3.1. For every parabolic subgroup $P$ with $P_{L} \subset P \subset G$ and Levi factor $M \supset L$, an algebra $\mathcal{H}^{M}$ and a Morita equivalence

$$
\Phi_{M}: \operatorname{Rep}(M)^{\mathfrak{s}_{M}} \rightarrow \operatorname{Mod}\left(\mathcal{H}^{M}\right)
$$

are given. When $P^{\prime} \supset P$ is another such parabolic subgroup, an algebra injection $\lambda_{M M^{\prime}}: \mathcal{H}^{M} \rightarrow \mathcal{H}^{M^{\prime}}$ is given, with the below properties.

(i) The following diagram commutes:

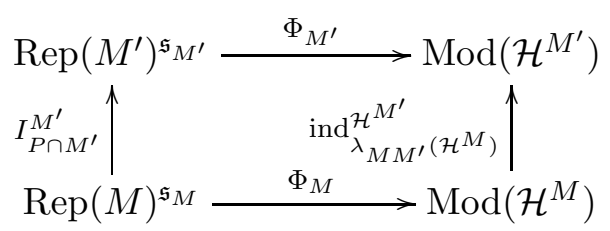

(ii) Let $\bar{P}$ be the parabolic subgroup of $G$ which has Levi factor $M$ and is opposite to $P$. Let $\operatorname{pr}_{\mathfrak{s}_{M}}: \operatorname{Rep}(M) \rightarrow \operatorname{Rep}(M)^{\mathfrak{s}_{M}}$ be the projection coming from the Bernstein decomposition for $M$. The following diagram commutes:

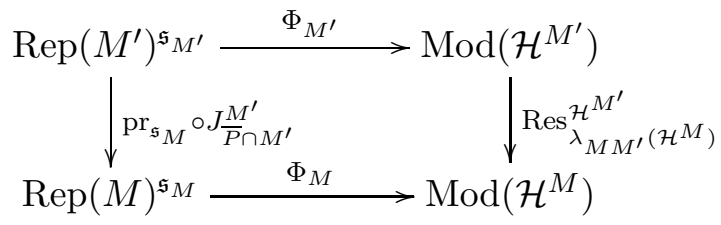

(iii) If $P \subset P^{\prime} \subset P^{\prime \prime} \subset G$, then $\lambda_{M M^{\prime \prime}}=\lambda_{M^{\prime} M^{\prime \prime}} \circ \lambda_{M M^{\prime}}$.

The Conditions 3.1 are quite general, in the sense that they do not involve the structure of the algebras $\mathcal{H}^{M}$. We will see later that in many cases these conditions hold already by abstract functoriality principles. 
The next series of conditions is much more specific though. For $P=M U$, let $R(M, L)$ be the set of roots of $M$ with respect to the maximal $F$-split torus $A_{L}$ in the centre of $L$. This is a root system when $L$ is a minimal $F$-Levi subgroup of $G$. In general it is only an orthogonal projection of such a root system (but in many cases encountered in the literature it is nevertheless a root system). For $P \supset P_{L}$ we define the set of positive roots as $R^{+}(M, L)=R\left(M \cap P_{L}, L\right)$, and we call the minimal elements of this set the simple roots of $(M, L)$.

Condition 3.2. Assume Condition 3.1.

(i) $\mathcal{H}^{G}$ (or $\left(\mathcal{H}^{G}\right)^{\text {op }}$ ) is an extended affine Hecke algebra $\mathcal{H}(\mathcal{R}, q) \rtimes \Gamma$.

(ii) All the $\mathcal{H}^{M}$ (or all the $\left(\mathcal{H}^{M}\right)^{\text {op }}$ ) are parabolic subalgebras and the $\lambda_{M M^{\prime}}$ are inclusions of parabolic subalgebras.

(iii) Consider the bijection

$$
\Phi_{L}: X_{\mathrm{nr}}(L) / X_{\mathrm{nr}}(L, \sigma) \cong \operatorname{Irr}(L)^{\mathfrak{s}_{L}} \rightarrow \operatorname{Irr}\left(\mathcal{H}^{L}\right) \cong T
$$

and its differential $\mathrm{d} \Phi_{L}: X^{*}(L) \otimes_{\mathbb{Z}} \mathbb{C} \rightarrow Y \otimes_{\mathbb{Z}} \mathbb{C}$.

Then $\mathrm{d} \Phi_{L}^{-1}$ maps the positive coroots $R^{\vee+}$ for $\mathcal{H}(\mathcal{R}, q)$ to $R^{+}(G, L)$, and $\mathrm{d} \Phi_{L}^{-1}\left(\mathbb{Q} R^{\vee}\right)$ has a $\mathbb{Q}$-basis consisting of simple roots of $(G, L)$.

(iv) Suppose that $Q \subset \Delta$ and $\mathrm{d} \Phi_{L}(\mathbb{Q} R(M, L)) \cap R^{\vee}=R_{Q}^{\vee}$. Then $\mathcal{H}^{M}=\mathcal{H}^{Q} \rtimes \Gamma_{M}$ for some $\Gamma_{M} \subset \Gamma(Q, Q)$. If moreover $\mathrm{d} \Phi_{L}(\mathbb{Q} R(M, L))=\mathbb{Q} Q^{\vee}$, then $\Gamma_{M}$ satisfies Condition 1.1 for $Q$.

In practice the positivity part of Condition 3.2.iii is innocent. Namely, usually one starts by fixing a minimal parabolic subgroup, and proves statements with that parabolic as the standard one. Suppose that $R(G, L)$ is a root system and that all the above conditions hold, except the positivity part of Condition 3.2, iii. Then $\mathrm{d} \Phi_{L}^{-1}\left(\mathbb{Q} R^{\vee}\right) \cap R(G, L)$ is a parabolic root subsystem of $R(G, L)$, so it is conjugate under $W(G, L)$ to a standard parabolic root subsystem, say $R(M, L)$. Applying an element of $W(M, L)$, we can moreover arrange that the image of $R^{\vee+}$ consists of positive roots. Equivalently, with respect to a different parabolic subgroup $P_{L}^{\prime}$ of $G$ with Levi factor $L$, Condition 3.2 iii is fulfilled.

Then we restart the whole procedure with $P_{L}^{\prime}$ instead of $P_{L}$, and the same arguments as before will also prove the required positivity statements. This applies to all the examples discussed in Sections 4 and 5 .

We draw some first consequences for the above conditions.

Lemma 3.3. Assume Conditions 3.1] and 3.2.

(a) There exists a canonical surjective homomorphism of complex tori $\Phi_{\mathrm{nr}}: X_{\mathrm{nr}}(L) \rightarrow$ $T$, with finite kernel $X_{\mathrm{nr}}(L, \sigma)$.

(b) When $d \Phi_{L}(\mathbb{Q} R(M, L)) \cap R^{\vee}=R_{Q}^{\vee}$, the image of $X_{\mathrm{nr}}(M)$ under the map from part (a) is contained in $T^{Q}$. When moreover $d \Phi_{L}(\mathbb{Q} R(M, L))=\mathbb{Q} Q^{\vee}$, the image of $X_{\mathrm{nr}}(M)$ equals $T^{Q}$.

(c) For all $\omega \in \operatorname{Rep}(M)^{\mathfrak{s}_{M}}$ and $\chi \in X_{\mathrm{nr}}(M): \Phi_{M}(\omega \otimes \chi)=\Phi_{M}(\omega) \otimes \Phi_{\mathrm{nr}}(\chi)$.

Proof. (a) The map $\chi \mapsto \sigma \otimes \chi$ induces an isomorphism of algebraic varieties

$$
X_{\mathrm{nr}}(L) / X_{\mathrm{nr}}(L, \sigma) \rightarrow \operatorname{Irr}(L)^{\mathfrak{s}_{L}}=\left\{\sigma \otimes \chi: \chi \in X_{\mathrm{nr}}(L)\right\} .
$$

By Condition 3.2. (ii) $\Phi_{L}$ gives a bijection $\operatorname{Irr}(L)^{\mathfrak{s}_{L}} \rightarrow \operatorname{Irr}\left(\mathcal{H}^{L}\right)=T$. This lifts to a surjective group homomorphism

$$
\Phi_{\mathrm{nr}}: X_{\mathrm{nr}}(L) \rightarrow T \quad \text { with } \quad \Phi_{L}(\sigma \otimes \chi)=\Phi_{L}(\sigma) \otimes \Phi_{\mathrm{nr}}(\chi) .
$$


(b) For every $Q \subset \Delta$ we defined the subtorus

$$
T^{Q}=\{t \in T: t(x)=1 \forall x \in \mathbb{Q} Q \cap X\}
$$

of $T$. Using Condition 3.2 . (iv) we can write

$$
X_{\mathrm{nr}}(M)=\left\{\chi \in X_{\mathrm{nr}}(L): \chi=1 \text { on } \mathbb{Q} R(M, L)^{\vee} \cap X^{*}\left(X_{\mathrm{nr}}(L)\right)\right\} .
$$

The relation between $M$ and $Q$ shows that the preimage of $\Phi_{\mathrm{nr}}^{-1}\left(T^{Q}\right)$ contains $X_{\mathrm{nr}}(M)$. When $\mathrm{d} \Phi_{L}(\mathbb{Q} R(M, L))=\mathbb{Q} Q^{\vee}, \Phi_{L}$ also induces a bijection between $\mathbb{Q} R(M, L)^{\vee}$ and $\mathbb{Q} Q$, and $X_{\mathrm{nr}}(M)$ is the full preimage of $T^{Q}$.

(c) The kernel of $\Phi_{\mathrm{nr}}: X_{\mathrm{nr}}(M) \rightarrow T^{Q}$ is

$$
X_{\mathrm{nr}}(M, \sigma):=X_{\mathrm{nr}}(L, \sigma) \cap X_{\mathrm{nr}}(M) .
$$

Then $X_{\mathrm{nr}}(M, \sigma)$ acts on $X_{\mathrm{nr}}(M)$ by translations and $\mathcal{G}_{Q, \sigma}$ acts on $T^{Q} \cong$ $X_{\mathrm{nr}}(M) / X_{\mathrm{nr}}(M, \sigma)$. By Lemma 2.3 there exists a canonical short exact sequence

$$
1 \rightarrow X_{\mathrm{nr}}(M, \sigma) \rightarrow \mathcal{G}_{Q, \sigma}^{\prime} \rightarrow \mathcal{G}_{Q, \sigma} \rightarrow 1,
$$

such that the action of $\mathcal{G}_{Q, \sigma}^{\prime}$ on $X_{\mathrm{nr}}(M)$ lifts that of $\mathcal{G}_{Q, \sigma}$ on $T^{Q}$. For $\omega \in \operatorname{Rep}(M)^{\mathfrak{s}_{M}}$ Condition 3.1. (ii) and (61) imply that

$$
\begin{aligned}
& \operatorname{Res}_{\lambda_{L M}\left(\mathcal{H}^{L}\right)}^{\mathcal{H}^{M}}\left(\Phi_{M}(\omega \otimes \chi)\right)=\Phi_{L}\left(J \frac{M}{P_{L} \cap M}(\omega \otimes \chi)\right) \\
& =\Phi_{L}\left(J \frac{M}{P_{L} \cap M}(\omega) \otimes \chi\right)=\Phi_{L}\left(J \frac{M}{P_{L} \cap M}(\omega)\right) \otimes \Phi_{L}(\chi) \\
& =\operatorname{Res}_{\lambda_{L M}^{M}\left(\mathcal{H}^{L}\right)}^{\mathcal{H}^{M}}\left(\Phi_{M}(\omega)\right) \otimes \Phi_{\mathrm{nr}}(\chi)=\operatorname{Res}_{\lambda_{L M}\left(\mathcal{H}^{L}\right)}^{\mathcal{H}^{M}}\left(\Phi_{M}(\omega) \otimes \Phi_{\mathrm{nr}}(\chi)\right) .
\end{aligned}
$$

When $\omega$ is irreducible, $\Phi_{M}(\omega \otimes \chi)$ lies in the same connected component of $\operatorname{Irr}\left(\mathcal{H}^{M}\right)$ as $\Phi_{M}(\omega)$, so (63) shows that it is an unramified twist of $\Phi_{M}(\omega)$. Hence

$$
\Phi_{M}(\omega \otimes \chi)=\Phi_{M}(\omega) \otimes \Phi_{\mathrm{nr}}(\chi) \quad \text { when } \omega \text { is irreducible. }
$$

Using the invertibility of $\Phi_{M}$, both sides of (64) define a group action of $X_{\mathrm{nr}}(M)$ on $\operatorname{Mod}\left(\mathcal{H}^{M}\right)$, by exact functors with commute with inductive (and projective) limits. Since these actions agree on irreducible representations, they agree on all representations.

Lemma 3.4. Assume Conditions 3.1 and 3.2 and suppose that $d \Phi_{L}(\mathbb{Q} R(M, L)) \cap R^{\vee}=R_{Q}^{\vee}$.

(a) The map $\Phi_{L}$ induces a group isomorphism $W_{\mathfrak{s}_{M}} \rightarrow W\left(R_{Q}\right) \Gamma_{M}$.

(b) $W_{\mathfrak{s}_{M}}$ fixes $X_{\mathrm{nr}}(M)$ pointwise and, when moreover $\mathrm{d} \Phi_{L}(\mathbb{Q} R(M, L))=\mathbb{Q} Q^{\vee}$, $W\left(R_{Q}\right) \Gamma_{M}$ fixes $T^{Q}$ pointwise.

Proof. (a) It suffices to prove this when $M=G$. By [BeDe, Théorème 2.13], the centre of the category $\operatorname{Rep}(G)^{\mathfrak{s}}$ is

$$
\mathcal{O}\left(\operatorname{Irr}(L)^{\mathfrak{s}_{L}}\right)^{W_{\mathfrak{s}}}=\mathcal{O}\left(\operatorname{Irr}(L)^{\mathfrak{s} L} / W_{\mathfrak{s}}\right) .
$$

The pointwise fixator of $X_{\mathrm{nr}}(L)$ in $N_{G}(L)$ is $Z_{G}\left(A_{L}\right)=Z_{G}\left(Z(L)^{\circ}\right)=L$. Since $W_{\mathfrak{s}} \subset N_{G}(L) / L$, it acts faithfully on $X_{\mathrm{nr}}(L)$ by algebraic group automorphisms. Hence $W_{\mathfrak{s}}$ also acts faithfully on $\operatorname{Irr}(L)^{\mathfrak{s}_{L}}$.

By (13) the centre of $\operatorname{Mod}(\mathcal{H} \rtimes \Gamma)$ is

$$
Z(\mathcal{H} \rtimes \Gamma)=\mathcal{O}(T)^{W \Gamma}=\mathcal{O}(T / W \Gamma),
$$

provided that $W \Gamma$ acts faithfully on $T$. Clearly $W$ acts faithfully on $T$. By assumption every $\gamma \in \Gamma$ acts on $R$ by a diagram automorphism, so it cannot act on $T$ as any 
nontrivial element of $W$. Hence, to check that $W \Gamma$ acts faithfully on $T$, it suffices to do so for $\Gamma$.

In view of (15), the isomorphism $\Phi_{L}: \operatorname{Irr}(L)^{\mathfrak{s}_{L}} \rightarrow T$ implies that $\Gamma_{\emptyset}$ is trivial. We recall from [Sau, Théorème 3.2] that that $I_{P_{L}}^{G}(\sigma \otimes \chi)$ is irreducible for $\chi$ in a Zariski-open nonempty subset of $X_{\mathrm{nr}}(L)$. If $\gamma \in \Gamma \backslash\{1\}$ would act trivially on $T$, then so would the cyclic group $\langle\gamma\rangle$ generated by it. In that case

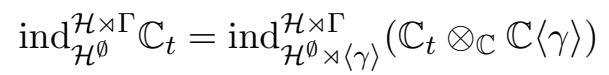

would be reducible for all $t \in T$ (as $\mathbb{C}\langle\gamma\rangle$ is reducible). That would contradict Condition 3.1.i. So $W \Gamma$ acts faithfully on $T$ and (65) holds.

Now Condition 3.1 says that

$$
Z(\mathcal{H} \rtimes \Gamma)=\mathcal{O}(T / W \Gamma) \cong \mathcal{O}\left(\operatorname{Irr}(L)^{\mathfrak{s}_{L}} / W_{\mathfrak{s}}\right) .
$$

From this and Condition 3.1,i, we deduce that $\Phi_{L}: \operatorname{Irr}(L)^{\mathfrak{S}_{L}} \rightarrow T$ induces a bijection

$$
\operatorname{Irr}(L)^{\mathfrak{s} L} / W_{\mathfrak{s}} \rightarrow T / W \Gamma .
$$

On both sides the finite groups act faithfully by automorphisms of complex algebraic varieties. Consider the open subvariety of $T$ (resp. of $\left.\operatorname{Irr}(L)^{\mathfrak{s}_{L}}\right)$ where the stabilizers in $W \Gamma$ (resp. in $W_{\mathfrak{s}}$ ) are trivial. For such a $t \in T$ and $\gamma \in W \Gamma$, the equation $\Phi_{L}\left(w \Phi_{L}^{-1} t\right)=\gamma t$ holds for a unique $w \in W_{\mathfrak{s}}$. This defines the group isomorphism $W \Gamma \rightarrow W_{\mathfrak{s}}$.

(b) The first claim is trivial, because $W_{\mathfrak{s}_{M}} \subset N_{M}(L) / L$. The second claim follows directly from the first claim, part (a) and Lemma 3.3. b.

Lemma 3.5. Assume

$$
M \mapsto \mathrm{d} \Phi_{L}(\mathbb{Q} R(M, L)) \cap \Delta^{\vee}
$$

induces a bijection between:

- $W_{\mathfrak{s}}$-association classes of Levi subgroups $M \subset G$ such that $L \subset M$ and $d \Phi_{L}(\mathbb{Q} R(M, L))$ equals the $\mathbb{Q}$-span of a subset of $\Delta^{\vee}$;

- subsets of $\Delta^{\vee}$, modulo $\Gamma W$-association.

Proof. Suppose that $M$ and $M^{\prime}$ are such Levi subgroups, and that they are conjugate by an element $w \in W_{\mathfrak{s}}$. Then the functors $I_{P}^{G}: \operatorname{Rep}(M)^{\mathfrak{s}_{M}} \rightarrow \operatorname{Rep}(G)^{\mathfrak{s}}$ and $I_{P^{\prime}}^{G}$ : $\operatorname{Rep}\left(M^{\prime}\right)^{\mathfrak{s}_{M^{\prime}}} \rightarrow \operatorname{Rep}(G)^{\mathfrak{s}}$ have the same image, for

$$
I_{P^{\prime}}^{G} \circ \operatorname{Ad}(w)^{*}=\operatorname{Ad}(w)^{*} \circ I_{P}^{G} \cong I_{P}^{G} .
$$

With Condition 3.1,i, this implies that $\operatorname{ind}_{\lambda_{M G}\left(\mathcal{H}^{M}\right)}^{\mathcal{H}^{G}}$ and $\operatorname{ind}_{\lambda_{M^{\prime} G}\left(\mathcal{H}^{M^{\prime}}\right)}^{\mathcal{H}^{G}}$ have the same image. Condition 3.2, ii says that $\mathcal{H}^{M}=\mathcal{H}^{Q} \rtimes \Gamma_{M}$ and $\mathcal{H}^{M^{\prime}}=\mathcal{H}^{Q^{\prime}} \rtimes \Gamma_{M^{\prime}}$ are parabolic subalgebras of $\mathcal{H}^{G}$, and then Proposition 1.6 shows that they must be $\mathcal{G}$-associate. Condition 3.2.iv implies that

$$
Q^{\vee}=\mathrm{d} \Phi_{L}(\mathbb{Q} R(M, L)) \cap \Delta^{\vee} \quad \text { and } \quad Q^{\prime \vee}=\mathrm{d} \Phi_{L}\left(\mathbb{Q} R\left(M^{\prime}, L\right)\right) \cap \Delta^{\vee}
$$

are $W \Gamma$-associate. Hence the map of the lemma is well-defined on the given equivalence classes.

Using the same notations as above, suppose that $Q^{\vee}$ and $Q^{\prime \vee}$ are $W \Gamma$-associate. Then $\mathrm{d} \Phi_{L}(\mathbb{Q} R(M, L))=\mathbb{Q} Q^{\vee}$ is $W \Gamma$-associate to $\mathrm{d} \Phi_{L}\left(\mathbb{Q} R\left(M^{\prime}, L\right)\right)=\mathbb{Q} Q^{\prime \vee}$. By Lemma 3.4, $\mathbb{Q} R(M, L)$ is $W_{\mathfrak{s}}$-associate to $\mathbb{Q} R\left(M^{\prime}, L\right)$. Hence $M$ and $M^{\prime}$ are conjugate by an element of $W_{\mathfrak{s}}$, showing that the map of the lemma is injective. 
By Condition 3.2.iii, the subgroup $P_{\mathfrak{s}} \subset G$ generated by $P_{L}$ and the root subgroups for roots in $\mathrm{d} \Phi_{L}^{-1}\left(\mathbb{Q} R^{\vee}\right)$ is a parabolic subgroup of $G$. The map of the statement sends the standard Levi factor $M_{\mathfrak{s}}$ of $P_{\mathfrak{s}}$ to $\Delta^{\vee}$.

Suppose that the map is not surjective. Choose $\tilde{Q}^{\vee} \subset \Delta^{\vee}$ which does not lie in the image and is maximal for that property. Since $\Delta^{\vee}$ belongs to the image, $\tilde{Q} \neq \Delta$ and we can find $\alpha \in \Delta \backslash \tilde{Q}$ such that $Q^{\vee}:=\tilde{Q}^{\vee} \cup\left\{\alpha^{\vee}\right\}$ does lie in the image. We write $\Gamma_{\tilde{Q}}=\Gamma_{Q} \cap \Gamma(\tilde{Q}, \tilde{Q})$.

For every Levi subgroup $M^{\prime} \subset M$ we choose finitely many representations $\pi_{M^{\prime}, i} \in$ $\operatorname{Irr}\left(M^{\prime}\right)^{\mathfrak{s}_{M^{\prime}}}$ as in Theorem 2.2, Then the representations

$$
I_{P^{\prime} \cap M}^{M}\left(\pi_{M^{\prime}, i} \otimes \chi_{M^{\prime}}\right) \quad \text { with } \chi_{M^{\prime}} \in X_{\mathrm{nr}}\left(M^{\prime}\right),
$$

for all $M^{\prime}$ and all possible $i$, span the Grothendieck group of $\operatorname{Rep}(M)^{\mathfrak{s}_{M}}$. Applying Condition 3.1, we find that the representations

$$
\operatorname{ind}_{\lambda_{M^{\prime} M} \mathcal{H}^{M}}^{\left.\mathcal{H}^{M^{\prime}}\right)} \Phi_{M^{\prime}}\left(\pi_{M^{\prime}, i} \otimes \chi_{M^{\prime}}\right)
$$

span the Grothendieck group $\operatorname{Gr}\left(\mathcal{H}^{M}\right)$ of $\operatorname{Mod}\left(\mathcal{H}^{M}\right)$. By Lemma 3.3

$$
\Phi_{M^{\prime}}\left(\pi_{M^{\prime}, i} \otimes \chi_{M^{\prime}}\right)=\Phi_{M^{\prime}}\left(\pi_{M^{\prime}, i}\right) \otimes \Phi_{\mathrm{nr}}\left(\chi_{M^{\prime}}\right)
$$

and $\Phi_{\mathrm{nr}}\left(\chi_{M^{\prime}}\right) \in T^{Q^{\prime}}$. For $M^{\prime} \neq M$ and $t \in T^{Q^{\prime}}, \Phi_{M^{\prime}}\left(\pi_{M^{\prime}, i}\right) \otimes t$ is a representation of $\mathcal{H}^{Q^{\prime}} \rtimes \Gamma_{M^{\prime}}$ with $Q^{\prime}$ not $W \Gamma$-associate to $\tilde{Q}$. Hence the collection of representations

$$
\Phi_{M}\left(\pi_{M, i}\right) \otimes t \quad \text { with } t \in T^{Q}
$$

spans the quotient

$$
\operatorname{Gr}\left(\mathcal{H}^{Q} \rtimes \Gamma_{M}\right) / \sum_{Q^{\prime} \subsetneq Q, Q^{\prime}} \operatorname{ind}_{\lambda_{M^{\prime} M}\left(\mathcal{H}^{Q^{\prime} \rtimes \Gamma_{M^{\prime}}}\right)}^{\mathcal{H}^{Q} \rtimes \Gamma_{M}} \operatorname{Gr}\left(\mathcal{H}^{Q^{\prime}} \rtimes \Gamma_{M^{\prime}}\right) .
$$

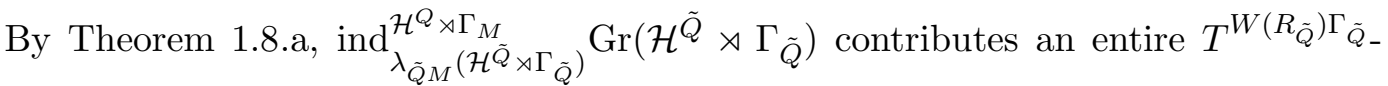
orbit of representations to (68).

By Lemma $3.4 T^{Q} \subset T^{W\left(R_{Q}\right) \Gamma_{Q}}$, which shows in particular that the translation part $z_{\gamma}$ of $\gamma$ is trivial for all $\gamma \in \Gamma_{Q}$. As $W\left(R_{\tilde{Q}}\right) \Gamma_{\tilde{Q}} \subsetneq W\left(R_{Q}\right) \Gamma_{Q}$, we have

$$
T^{W\left(R_{\tilde{Q}}\right) \Gamma_{\tilde{Q}} \supset T^{Q} .}
$$

We want to see that the left hand side has higher dimension than the right hand side. By construction $W\left(R_{\tilde{Q}}\right)$ fixes $T^{\tilde{Q}}$ pointwise. The torus $T_{1}=\left(T_{Q} \cap T^{\tilde{Q}}\right)^{\circ}$ is one-dimensional, because $|Q \backslash \tilde{Q}|=1$. For the same reason $\alpha: T_{1} \rightarrow \mathbb{C}^{\times}$is a surjection with finite kernel. The group $\Gamma_{\tilde{Q}}=\Gamma_{Q} \cap \Gamma(\tilde{Q}, \tilde{Q})$ stabilizes both $Q$ and $\tilde{Q}$, so fixes $\alpha$. Therefore $\Gamma_{\tilde{Q}}$ fixes $T_{1}$ pointwise. It follows that $W\left(R_{\tilde{Q}}\right) \Gamma_{\tilde{Q}}$ fixes the torus $T^{\tilde{Q}}=T^{Q} T_{1}$ pointwise.

Returning to (67) and (68), we see now that the contribution from $\mathcal{H}^{\tilde{Q}} \rtimes \Gamma_{\tilde{Q}}$ encompasses at least one $T^{\tilde{Q}}$-orbit. But that is impossible, because the $i$ 's in (67) belong to a finite set and $\operatorname{dim}_{\mathbb{C}}\left(T^{Q^{\prime}}\right)>\operatorname{dim}_{\mathbb{C}}\left(T^{Q}\right)$. This contradiction entails that the map from the statement is surjective. 


\subsection{Preservation of temperedness.}

We will show that the above conditions imply that the Morita equivalences preserve temperedness and (under an extra condition) discrete series. For $\Phi_{M}^{-1}$ this is relatively easy.

Lemma 3.6. Assume Conditions 3.1 and [3.2, and let $P=M U$ be a parabolic subgroup containing $P_{L}$ such that $d \Phi_{L}(\mathbb{Q} R(M, L))=\mathbb{Q} Q^{\vee}$.

(a) $\Phi_{M}^{-1}$ preserves temperedness of finite length representations.

(b) $\Phi_{M}^{-1}$ sends finite dimensional essentially discrete series $\mathcal{H}^{M}$-representations to essentially square-integrable $M$-representations.

(c) Suppose that $\pi^{\prime} \in \operatorname{Mod}\left(\mathcal{H}^{M}\right)$ has finite dimension, is tempered, essentially discrete series and factors through $\psi_{t}: \mathcal{H}^{Q} \rtimes \Gamma_{M} \rightarrow \mathcal{H}_{Q} \rtimes \Gamma_{M}$ for some $t \in T^{Q}$. Then the $M$-representation $\Phi_{M}^{-1}\left(\pi^{\prime}\right)$ is square-integrable modulo centre.

Proof. (a) Since every irreducible $\mathcal{H}^{M}$-module has finite dimension, $\Phi_{M}$ restricts to an equivalence between finite length representations on the one hand, and the finite dimensional modules on the other hand.

Let $\pi \in \operatorname{Rep}(M)^{\mathfrak{s}_{M}}$ be of finite length, and recall the criterion for temperedness from [Wal, Proposition III.2.2] and (43). As the supercuspidal support of $\pi$ is contained in $[L, \sigma]_{G}$, it is equivalent to impose these conditions only with respect to the parabolic subgroup $P_{L}=L U_{L}$ [Hei2, Proposition 1.2.i]. Let $\overline{P_{L}}$ be the parabolic subgroup opposite to $P_{L}$. Then $\overline{P_{L}} \cap M$ is opposite to $P_{L} \cap M$. The above condition (for $P_{L}$ ) is equivalent to:

$$
\log |\chi| \in \overline{{ }^{+a^{M}} P_{L} \cap M}=\left\{\left.\sum_{\alpha \in R\left(P_{L} \cap M, L\right)} c_{\alpha} \alpha\right|_{A_{L}}: c_{\alpha} \leq 0\right\}
$$

for every $A_{L}$-weight $\chi$ of $J_{M \cap \overline{P_{L}}}^{M} \pi$.

By the assumption on $M$ and Condition 3.2. iii, $\mathrm{d} \Phi_{L}^{-1}: \mathbb{Q} Q^{\vee} \rightarrow \mathbb{Q} R(M, L)$ is a linear bijection which sends

$$
\mathfrak{a}^{-Q}=\left\{\sum_{\alpha \in Q} \lambda_{\alpha} \alpha^{\vee}: \lambda_{\alpha} \leq 0\right\} \quad \text { to } \overline{{ }^{+} \mathfrak{a}_{\bar{P}}{ }_{\bar{L}} \cap M} .
$$

Suppose that $\Phi_{M}(\pi)$ is tempered. By definition, this means that all $\mathcal{H}^{L}$-weights $t$ of $\operatorname{Res}_{\lambda_{L M}\left(\mathcal{H}^{L}\right)}^{\mathcal{H}^{M}}\left(\Phi_{M}(\pi)\right)$ satisfy $\log |t| \in \mathfrak{a}^{-Q}$. By Condition 3.1.(ii) and (70), all $A_{L}$-weights $\chi$ of $J_{M \cap \overline{P_{L}}}^{M} \pi$ have $\log |\chi| \in \overline{{ }^{+\mathfrak{a}^{M}} \overline{P_{L}} \cap M}$. Thus (69) says that $\pi$ is tempered. (b) By Wal, Proposition III.1.1] and arguments analogous to the above, $\pi$ is squareintegrable modulo centre if and only if

$$
\log |\chi| \in{ }^{+} \mathfrak{a}_{P_{L} \cap M}^{M}=\left\{\left.\sum_{\alpha \in R\left(P_{L} \cap M, L\right)} c_{\alpha} \alpha\right|_{A_{L}}: c_{\alpha}<0\right\}
$$

for every $A_{L}$-weight $\chi$ of $J_{M \cap \overline{P_{L}}}^{M} \pi$. The criterium for essential square-integrability then becomes

$$
\log |\chi| \in{ }^{+} \mathfrak{a} \frac{M}{P_{L} \cap M}+\mathfrak{a}_{M} .
$$

for every $A_{L}$-weight $\chi$ of $J_{M \cap \bar{P}_{L}}^{M} \pi$. Since the rank $|Q|$ of $R_{Q}$ equals the rank of $R(M, L)$ and $\mathrm{d} \Phi_{L}^{-1}$ preserves positivity, it maps $\mathfrak{a}^{--Q}$ (the interior of $\mathfrak{a}^{-Q}$ ) to $\mathfrak{a}_{P_{L}}^{M} M$. By Lemma 3.3.b $\Phi_{L}^{-1}\left(T^{Q}\right)=X_{\mathrm{nr}}(M)$.

Suppose that $\Phi_{M}(\pi)$ is essentially discrete series. By definition, this means that all $\mathcal{H}^{L}$-weights $t$ of $\operatorname{Res}_{\lambda_{L M}\left(\mathcal{H}^{L}\right)}^{\mathcal{H}^{M}}\left(\Phi_{M}(\pi)\right)$ satisfy $|t| \in \exp \left(\mathfrak{a}^{--Q}\right) T^{Q}$. By the above 
and Condition 3.1,ii, all $A_{L}$-weights of $J_{M \cap \bar{P}_{L}}^{M} \pi$ lie in (72). Hence $\pi$ is essentially square-integrable.

(c) Recall that a $M$-representation is essentially square-integrable if its restriction to the derived subgroup of $M$ is square-integrable. If a $M$-representation with a central character is tempered, then $Z(M)$ acts on its by a unitary character. Hence all tempered essentially square-integrable representations with a central character are square-integrable modulo centre.

By parts (a) and (b) $\Phi_{M}^{-1}\left(\pi^{\prime}\right)$ is tempered and essentially square-integrable. Since $\Phi_{M}^{-1}\left(\pi^{\prime}\right)$ lies in one Bernstein component $\operatorname{Rep}(M)^{\mathfrak{s}_{M}}$, the maximal compact subgroup of $Z(M)$ acts on it by a single character $\chi_{0}$, which is automatically unitary. Then $X_{\mathrm{nr}}(M)$ (resp. $X_{\mathrm{unr}}(M)$ ) parametrizes the extensions of $\chi_{0}$ to a character (resp. unitary character) of $Z(M)$. Lemma 3.3. says that $Z(M)$ acts on $\Phi_{M}^{-1}\left(\pi^{\prime}\right)$ by the character determined by $\chi_{0}$ and $\Phi_{\mathrm{nr}}^{-1}(t)$. Lemma 1.3. b shows that $t \in T_{\mathrm{un}}^{Q}$ and $\Phi_{\mathrm{nr}}^{-1}\left(T_{\mathrm{un}}\right)=X_{\mathrm{unr}}(L)$, so $Z(M)$ acts by a unitary character.

Part (a) of Lemma 3.6 admits a quick generalization to all Levi subgroups that we encounter. On the other hand, that is not possible for parts (b) and (c). In fact, for Levi subgroups $M \subset G$ containing $L$ but not of the form as in Lemma 3.6. $\operatorname{Rep}(M)^{\mathfrak{s}_{M}}$ contains no essentially square-integrable representations. We delay the proof of that claim to Proposition 3.10. We note that in those cases $\operatorname{Irr}_{L^{2}}\left(\mathcal{H}^{M}\right)$ can still be nonempty.

Lemma 3.7. Assume Conditions 3.1 and 3.2, and let $P^{\prime}=M^{\prime} U^{\prime}$ be a parabolic subgroup containing $P_{L}$ such that $\mathbb{Q} R\left(M^{\prime}, L\right) \cap \mathrm{d} \Phi_{L}^{-1}\left(R^{\vee}\right)$ does not span $\mathbb{Q} R\left(M^{\prime}, L\right)$. Then $\Phi_{M^{\prime}}^{-1}$ preserves temperedness of finite length representations.

Proof. By Condition 3.2. iii $\mathrm{d} \Phi_{L}\left(\mathbb{Q} R\left(M^{\prime}, L\right)\right) \cap R^{\vee}$ is a standard parabolic root subsystem of $R^{\vee}$, that is, of the form $R_{Q}^{\vee}$ for a unique $Q \subset \Delta$. By Lemma 3.5 there exists a Levi subgroup $M \subset M^{\prime}$ such that $L \subset M$ and $\mathrm{d} \Phi_{L}(\mathbb{Q} R(M, L))=\mathbb{Q} Q^{\vee}$.

By Condition 3.2, iv $\mathcal{H}^{M^{\prime}}=\mathcal{H}^{Q} \rtimes \Gamma_{M^{\prime}}$ and $\mathcal{H}^{M}=\mathcal{H}^{Q} \rtimes \Gamma_{M}$, and by Condition 3.2, ii $\Gamma_{M^{\prime}} \supset \Gamma_{M}$ and $\lambda_{M M^{\prime}}$ is just the inclusion. The cone $T^{-Q} \subset T_{\mathrm{rs}}$ is the same for $\mathcal{H}^{M}, \mathcal{H}^{Q}$ and $\mathcal{H}^{M^{\prime}}$. For any finite dimensional $\mathcal{H}^{M}$-module $V$ :

$$
\mathrm{Wt}\left(\operatorname{ind}_{\mathcal{H}^{M}}^{\mathcal{H}^{M^{\prime}}}(V)\right)=\left\{\gamma(t): t \in \mathrm{Wt}(V), \gamma \in \Gamma_{M^{\prime}}\right\}
$$

Since $\Gamma_{M^{\prime}}$ preserves $T^{-Q}$, $\operatorname{ind}_{\mathcal{H}^{M}}^{\mathcal{H}^{M^{\prime}}}(V)$ is tempered if and only if $V$ is tempered. Similarly, (73) shows that a finite dimensional $\mathcal{H}^{M^{\prime}}$-module $V^{\prime}$ is tempered if and only if $\operatorname{Res}_{\mathcal{H}^{M}}^{\mathcal{H}^{M^{\prime}}}\left(V^{\prime}\right)$ is tempered. We note also that

$$
\operatorname{ind}_{\mathcal{H}^{M}}^{\mathcal{H}^{M^{\prime}}} \operatorname{Res}_{\mathcal{H}^{M}}^{\mathcal{H}^{M^{\prime}}}\left(V^{\prime}\right) \cong \mathbb{C}\left[\Gamma_{M^{\prime}}\right] \otimes_{\mathbb{C}\left[\Gamma_{M}\right]} V^{\prime} \cong \mathbb{C}\left[\Gamma_{M^{\prime}} / \Gamma_{M}\right] \otimes_{\mathbb{C}} V^{\prime},
$$

a $\mathcal{H}^{Q} \rtimes \Gamma_{M^{\prime}}$-module for the diagonal action. Then (74) contains $V^{\prime}$ as the direct summand $\mathbb{C}\left[\Gamma_{M^{\prime}} / \Gamma_{M^{\prime}}\right] \otimes_{\mathbb{C}} V^{\prime}$, and the restriction of (74) to $\mathcal{H}^{M}$ is a direct sum of copies of $\operatorname{Res}_{\mathcal{H}^{M}}^{\mathcal{H}^{M^{\prime}}}\left(V^{\prime}\right)$.

We recall from [Ren, Lemme VII.2.2] that $I_{P \cap M^{\prime}}^{M^{\prime}}$ always preserves temperedness. Consider a finite dimensional tempered module $V^{\prime} \in \operatorname{Mod}\left(\mathcal{H}^{M^{\prime}}\right)$. By Lemma 3.6. a the $M^{\prime}$-representation $I_{P \cap M^{\prime}}^{M^{\prime}} \circ \Phi_{M}^{-1} \circ \operatorname{Res}_{\mathcal{H}^{M}}^{\mathcal{H}^{M^{\prime}}}\left(V^{\prime}\right)$ is tempered. By Condition $3.1 \mathrm{it}$ is isomorphic to

$$
\Phi_{M^{\prime}}^{-1} \circ \operatorname{ind}_{\mathcal{H}^{M}}^{\mathcal{H}^{M^{\prime}}} \circ \operatorname{Res}_{\mathcal{H}^{M}}^{\mathcal{H}^{M^{\prime}}}\left(V^{\prime}\right) \cong I_{P \cap M^{\prime}}^{M^{\prime}} \circ \operatorname{pr}_{\mathfrak{s}_{M}} \circ J_{\bar{P} \cap M^{\prime}}^{M^{\prime}} \circ \Phi_{M^{\prime}}^{-1}\left(V^{\prime}\right),
$$


Since $\Phi_{M^{\prime}}^{-1}$ is an equivalence, (74) shows that (75) contains $\Phi_{M^{\prime}}^{-1}\left(V^{\prime}\right)$ as a direct summand. So the latter is tempered as well.

Let $M \supset L$ be a Levi subgroup of $G$ and write $\mathrm{d} \Phi_{L}(\mathbb{Q} R(M, L)) \cap R^{\vee}=R_{Q}^{\vee}$. As family of parabolic subalgebras of $\mathcal{H}^{M}$ we take $\mathcal{H}^{M}$ and the $\mathcal{H}^{M_{1}}$ where $M \supsetneq M_{1} \supset L$ and $\mathrm{d} \Phi_{L}\left(\mathbb{Q} R\left(M_{1}, L\right)\right)=\mathbb{Q} Q_{1}$ for some $Q_{1} \subsetneq Q$. By Lemma 3.5 every (proper) subset of $Q$ is obtained in this way. Recall the notion of Langlands constituents from Theorems 1.10 and 2.6 .

Lemma 3.8. Let $M^{\prime}$ be a Levi subgroup of $M$ containing $L$, such that $\mathrm{d} \Phi_{L}\left(\mathbb{Q} R\left(M^{\prime}, L\right)\right)=\mathbb{Q} Q^{\prime \vee}$ for a subset $Q^{\prime} \subset Q$. Let $\sigma \in \operatorname{Irr}_{L^{2}}\left(\mathcal{H}_{Q^{\prime}} \rtimes \Gamma_{M^{\prime}}\right)$ and $t \in T^{Q^{\prime}}$. Then

$$
\Phi_{M^{\prime}}^{-1}(\sigma \otimes t)=\Phi_{M^{\prime}}^{-1}\left(\sigma \otimes t|t|^{-1}\right) \otimes \Phi_{\mathrm{nr}}^{-1}(|t|) \quad \text { with } \quad \Phi_{M^{\prime}}^{-1}\left(\sigma \otimes t|t|^{-1}\right) \in \operatorname{Irr}_{L^{2}}\left(M^{\prime}\right)^{\mathfrak{s}_{M^{\prime}}} .
$$

The Morita equivalence $\Phi_{M}^{-1}$ restricts to a bijection between the Langlands constituents of $\operatorname{ind}_{\mathcal{H}^{M^{\prime}}}^{\mathcal{H}^{M}}(\sigma \otimes t)$ and those of

$$
\Phi_{M}^{-1}\left(\operatorname{ind}_{\mathcal{H}^{M^{\prime}}}^{\mathcal{H}^{M}}(\sigma \otimes t)\right) \cong I_{\left(M \cap P_{L}\right) M^{\prime}}^{M}\left(\Phi_{M^{\prime}}^{-1}\left(\sigma \otimes t|t|^{-1}\right) \otimes \Phi_{\mathrm{nr}}^{-1}(|t|)\right) .
$$

Remarks. By Lemma 3.3. a $\Phi_{\mathrm{nr}}$ becomes injective when restricted to unramified characters with values in $\mathbb{R}_{>0}$. Therefore $\Phi_{\mathrm{nr}}^{-1}(|t|) \in X_{\mathrm{nr}}\left(M^{\prime}\right)$ is well-defined.

When $Q^{\prime}=Q$, Langlands constituents are not defined on the Hecke algebra side. In that case the lemma must be interpreted differently. The functor $\operatorname{ind}_{\mathcal{H}^{M^{\prime}}}^{\mathcal{H}^{M}}=$ $\operatorname{ind}_{\mathcal{H}^{Q} \rtimes \Gamma_{M^{\prime}}}^{\mathcal{H}^{Q} \rtimes \Gamma_{M}}$ preserves complete reducibility (by Clifford theory, as $\Gamma_{M}$ is finite). By Condition 3.1. i, so does $I_{\left(P_{L} \cap M\right) M^{\prime}}^{M}$. In view of Proposition 2.7. b, the lemma becomes true in the case $Q^{\prime}=Q$, provided we declare that all irreducible subquotients of $\operatorname{ind}_{\mathcal{H}^{M^{\prime}}}^{\mathcal{H}^{M}}(\sigma \otimes t)$ are Langlands constituents.

Proof. The alternative expression for $\Phi_{M^{\prime}}^{-1}(\sigma \otimes t)$ comes from Lemma 3.3.c. By Lemma $1.3 \sigma \otimes t|t|^{-1} \in \operatorname{Irr}_{L^{2}}\left(\mathcal{H}^{Q^{\prime}} \rtimes \Gamma_{M^{\prime}}\right)$, and by Lemma 3.6. $c \Phi_{M^{\prime}}^{-1}\left(\sigma \otimes t|t|^{-1}\right) \in$ $\operatorname{Irr}_{L^{2}}\left(M^{\prime}\right)$.

Case I. Suppose that $\operatorname{ind}_{\mathcal{H}^{M^{\prime}}}^{\mathcal{H}^{M}}(\sigma \otimes t)$ is completely reducible. By Proposition 1.11.b all its irreducible subquotients are Langlands constituents. Since $\Phi_{M}$ is an equivalence, $\Phi_{M}^{-1}\left(\operatorname{ind}_{\mathcal{H}^{M^{\prime}}}^{\mathcal{H}^{M}}(\sigma \otimes t)\right)$ is also completely reducible. By Proposition 2.7.b all its irreducible subquotients are Langlands constituents. Hence $\Phi_{M}^{-1}$ provides a bijection between these two collections of Langlands constituents.

Case II. Suppose that ind $\mathcal{H}^{\mathcal{H}^{M}}(\sigma \otimes t)$ is not completely reducible. By Proposition 1.11.b there exists an $r \in \mathbb{R}_{>-1}$ such that $\operatorname{ind}_{\mathcal{H}^{M^{\prime}}}^{\mathcal{H}^{M}}\left(\sigma \otimes t|t|^{r}\right)$ is completely reducible. By Proposition 1.11,a, Case I and Proposition 2.7. a, the four representations

$$
\operatorname{ind}_{\mathcal{H}^{M^{\prime}}}^{\mathcal{H}^{M}}(\sigma \otimes t), \operatorname{ind}_{\mathcal{H}^{M^{\prime}}}^{\mathcal{H}^{M}}\left(\sigma \otimes t|t|^{r}\right), \Phi_{M}^{-1}\left(\operatorname{ind}_{\mathcal{H}^{M^{\prime}}}^{\mathcal{H}^{M}}\left(\sigma \otimes t|t|^{r}\right)\right) \text { and } \Phi_{M}^{-1}\left(\operatorname{ind}_{\mathcal{H}^{M^{\prime}}}^{\mathcal{H}^{M}}(\sigma \otimes t)\right)
$$

have the same number of inequivalent Langlands constituents.

Let $\pi^{\prime}$ be a non-Langlands constituent of $\operatorname{ind}_{\mathcal{H}^{M^{\prime}}}^{\mathcal{H}^{M}}(\sigma \otimes t)$. By Theorem 1.10, c there exists a Levi subgroup $M_{1} \subset M$, with $M_{1}=M$ or $\mathrm{d} \Phi_{L}\left(\mathbb{Q} R\left(M_{1}, L\right)\right)=\mathbb{Q} Q_{1}^{\vee} \subsetneq$ $Q^{\vee}$, such that $\pi^{\prime}$ is a constituent of $\operatorname{ind}_{\mathcal{H}^{M_{1}}}^{\mathcal{H}^{M}}\left(\sigma_{1} \otimes t_{1}\right)$ for some $t_{1} \in T^{Q_{1}}$ and $\sigma_{1} \in$ $\operatorname{Irr}_{L^{2}}\left(\mathcal{H}_{Q_{1}} \rtimes \Gamma_{M_{1}}\right)$ with $\left\|c c\left(\sigma_{1}\right)\right\|>\|c c(\sigma)\|$. When $M_{1}=M$, Lemma 1.3 shows that 
the same condition on $\pi^{\prime}$ is also fulfilled for the unique Levi subgroup $M_{2} \subset M$ with $\mathrm{d} \Phi_{L}\left(\mathbb{Q} R\left(M_{2}, L\right)\right)=\mathbb{Q} Q^{\vee}$. In that case we replace $M_{1}$ by $M_{2}$.

Now $\Phi_{M_{1}}^{-1}\left(\sigma_{1} \otimes t_{1}\left|t_{1}\right|^{-1}\right) \in \operatorname{Irr}_{L^{2}}\left(M_{1}\right)$ and $\pi^{\prime}$ is a constituent of

$$
I_{\left(M \cap P_{L}\right) M_{1}}^{M}\left(\Phi_{M^{\prime}}^{-1}\left(\sigma_{1} \otimes t_{1}\left|t_{1}\right|^{-1}\right) \otimes \Phi_{\mathrm{nr}}^{-1}\left(\left|t_{1}\right|\right)\right) .
$$

Recall that the invariant $\left\|c c_{M_{1}}\right\|$ on $\operatorname{Irr}\left(M_{1}\right)^{\mathfrak{s} M_{1}}$ is defined via a $W_{\mathfrak{s}}$-invariant inner product on $\mathfrak{a}_{L}=X^{*}\left(A_{L}\right) \otimes_{\mathbb{Z}} \mathbb{R}$. Via Lemmas 3.3. a and 3.4, a this can be transferred (canonically) to a $W \Gamma$-invariant inner product on $\mathfrak{a}$. The supercuspidal support (which is involved in $c c_{M_{1}}$ ) on $\operatorname{Irr}\left(M_{1}\right)^{\mathfrak{s}_{M_{1}}}$ is (up to conjugation) given by $J_{P_{L}}^{M_{1}} M_{1}$. Then Condition 3.1,ii shows that

$$
\begin{aligned}
\left\|c c_{M_{1}}\left(\Phi_{M_{1}}^{-1}\left(\sigma_{1} \otimes t_{1}\left|t_{1}\right|^{-1}\right)\right)\right\| & =\left\|c c\left(\sigma_{1} \otimes t_{1}\left|t_{1}\right|^{-1}\right)\right\|=\left\|c c\left(\sigma_{1}\right)\right\|> \\
\|c c(\sigma)\| & =\left\|c c\left(\sigma \otimes t|t|^{-1}\right)\right\|=\left\|c c_{M^{\prime}}\left(\Phi_{M^{\prime}}^{-1}\left(\sigma \otimes t|t|^{-1}\right)\right)\right\| .
\end{aligned}
$$

Now Theorem 2.6. c says that $\Phi_{M}^{-1}\left(\pi^{\prime}\right)$ is not a Langlands constituent of $\Phi_{M}^{-1}\left(\operatorname{ind}_{\mathcal{H}^{M^{\prime}}}^{\mathcal{H}^{M}}(\sigma \otimes t)\right)$. Summarizing, we know that:

- $\Phi_{M}^{-1}$ provides a bijection between the collections of inequivalent irreducible subquotients of ind $\mathcal{H}^{\mathcal{H}^{M}}(\sigma \otimes t)$ and of $\Phi_{M}^{-1}\left(\operatorname{ind}_{\mathcal{H}^{M^{\prime}}}^{\mathcal{H}^{M}}(\sigma \otimes t)\right)$;

- these two collections have the same number of Langlands constituents and the same number of non-Langlands constituents;

- $\Phi_{M}^{-1}$ maps non-Langlands constituents of $\operatorname{ind}_{\mathcal{H}^{M^{\prime}}}^{\mathcal{H}^{M}}(\sigma \otimes t)$ to non-Langlands constituents of $\Phi_{M}^{-1}\left(\operatorname{ind}_{\mathcal{H}^{M^{\prime}}}^{\mathcal{H}^{M}}(\sigma \otimes t)\right)$.

Consequently $\Phi_{M}^{-1}$ also provides a bijection between the collections of inequivalent Langlands constituents on both sides.

Now we are ready for the proof of main result of this paragraph.

Theorem 3.9. Assume Conditions 3.1 and 3.2, and let $P=M U$ be a parabolic subgroup containing $P_{L}$.

(a) $\Phi_{M}$ restricts to an equivalence between the category of finite length tempered representations in $\operatorname{Rep}(M)^{\mathfrak{s}_{M}}$ and the category of finite dimensional tempered $\mathcal{H}^{M}$-modules.

(b) Suppose that $d \Phi_{L}(\mathbb{Q} R(M, L))=\mathbb{Q} Q^{\vee}$ for some $Q \subset \Delta$. Then $\Phi_{M}$ sends finite length essentially square-integrable $M$-representations to essentially discrete series $\mathcal{H}^{M}$-representations, and $\Phi_{M}^{-1}$ does the converse.

Proof. (a) In view of Lemmas 3.6. a and 3.7, it suffices to prove that $\Phi_{M}$ preserves temperedness of finite length representations.

Suppose, on the contrary, that there exists a finite length tempered $\pi \in \operatorname{Rep}(M)^{\mathfrak{s}_{M}}$ such that $\Phi_{M}(\pi) \in \operatorname{Mod}\left(\mathcal{H}^{M}\right)$ is not tempered. Since $\Phi_{M}(\pi)$ has finite length, it has a composition series with finite dimensional irreducible quotients. It follows directly from the definition of temperedness for $\mathcal{H}^{M}=\mathcal{H}^{Q} \rtimes \Gamma_{M}$ that at least one of these irreducible subquotients, say $\rho_{1}$, is not tempered. Then we may replace $\Phi_{M}(\pi)$ by $\rho_{1}$ and $\pi$ by $\Phi_{M}^{-1}\left(\rho_{1}\right)$. Hence it suffices to prove the claim for irreducible representations.

We take the same family of parabolic subalgebras of $\mathcal{H}^{M}$ as in Lemma 3.8. By Theorem 1.10.c there exists a Levi subgroup $M_{1} \subset M$, with $M_{1}=M$ or $\mathrm{d} \Phi_{L}\left(\mathbb{Q} R\left(M_{1}, L\right)\right)=\mathbb{Q} Q_{1}^{\vee} \subsetneq Q^{\vee}$, such that $\Phi_{M}(\pi)$ is a Langlands constituent of 
$\operatorname{ind}_{\mathcal{H}^{M_{1}}}^{\mathcal{H}^{M}}\left(\sigma_{1} \otimes t_{1}\right)$ for some $t_{1} \in T^{Q_{1}}$ and $\sigma_{1} \in \operatorname{Irr}_{L^{2}}\left(\mathcal{H}_{Q_{1}} \rtimes \Gamma_{M_{1}}\right)$. When $M_{1}=M$, Lemma 1.3 shows that the same condition on $\pi^{\prime}$ is also fulfilled for the unique Levi subgroup $M_{2} \subset M$ with $\mathrm{d} \Phi_{L}\left(\mathbb{Q} R\left(M_{2}, L\right)\right)=\mathbb{Q} Q$. In that case we replace $M_{1}$ by $M_{2}$.

By Lemma $3.8 \pi$ is a Langlands constituent of

$$
\Phi_{M}^{-1}\left(\operatorname{ind}_{\mathcal{H}^{M_{1}}}^{\mathcal{H}^{M}}(\sigma \otimes t)\right) \cong I_{\left(M \cap P_{L}\right) M_{1}}^{M}\left(\Phi_{M_{1}}^{-1}\left(\sigma \otimes t|t|^{-1}\right) \otimes \Phi_{\mathrm{nr}}^{-1}(|t|)\right) .
$$

Suppose that $\Phi_{M}(\pi)$ is not tempered, so $t \notin T_{\text {un }}$ by Theorem 1.10, d. Then $\Phi_{\mathrm{nr}}^{-1}(|t|) \in$ $X_{\mathrm{nr}}\left(M^{\prime}\right) \backslash X_{\mathrm{unr}}\left(M^{\prime}\right)$, and by Theorem 2.6. $\mathrm{b} \pi$ is not tempered.

With Lemma 3.6. a we see that $\Phi_{M}^{-1}$ preserves both temperedness and non-temperedness of irreducible representations. Hence so does $\Phi_{M}$.

(b) For $\Phi_{M}^{-1}$ this is Lemma 3.6, b, so we only have to consider the claim for $\Phi_{M}$. Up to (78) we can follow the proof of part (a), only replacing tempered by essentially discrete series everywhere.

Suppose that $\Phi_{M}(\pi)$ is not essentially discrete series. By the uniqueness in Theorem 1.10. b $Q^{\prime}$ is a proper subset of $Q$. Then $M^{\prime}$ is a proper Levi subgroup of $M$, so by the uniqueness in Theorem 2.6. a $\pi$ is not essentially square-integrable. With Lemma 3.6. b we conclude that $\Phi_{M}^{-1}$ sends those irreducible representations which are essentially discrete series to essentially square-integrable representations, and those which are not essentially discrete series to representations that are not essentially square-integrable. Now it is clear that $\Phi_{M}$ also respects these properties.

For essentially square-integrable representations we can be more precise than Theorem 3.9. We write $\mathrm{d} \Phi_{L}(\mathbb{Q} R(M, L)) \cap R^{\vee}=R_{Q}^{\vee}$.

Proposition 3.10. Assume Conditions 3.1 and 3.2, and let $P=M U$ be a parabolic subgroup containing $P_{L}$.

(a) Suppose that $\mathbb{Q} R(M, L)) \cap \mathrm{d} \Phi_{L}^{-1}\left(R^{\vee}\right)$ does not span $\mathbb{Q} R(M, L)$. Then $\operatorname{Rep}(M)^{\mathfrak{s}_{M}}$ contains no finite length essentially square-integrable representations.

(b) Suppose that $\pi \in \operatorname{Rep}(M)^{\mathfrak{s}_{M}}$ is square-integrable modulo centre and has finite length. Then $\Phi_{M}(\pi)$ is tempered, essentially discrete series and factors through $\psi_{t}: \mathcal{H}^{Q} \rtimes \Gamma_{M} \rightarrow \mathcal{H}_{Q} \rtimes \Gamma_{M}$ for some $t \in T_{\mathrm{un}}^{Q}$.

(c) Suppose that $d \Phi_{L}(\mathbb{Q} R(M, L))=\mathbb{Q} Q^{\vee}$. Then $\Phi_{M}$ gives a bijection between $\operatorname{Irr}_{L^{2}}(M)^{\mathfrak{s}_{M}}$ and $\operatorname{Irr}_{L^{2}}\left(\mathcal{H}^{M}\right)$.

Proof. (a) Suppose, contrary to what we need to show, that $\operatorname{Rep}(M)^{\mathfrak{s}_{M}}$ does contain a representation of the indicated kind. Since it has finite length, it has an irreducible subrepresentation, say $\pi$. Let $\zeta$ be the central character of $\pi$, and let $|\zeta| \in X_{\mathrm{nr}}(M)$ be its absolute value. Then $\pi \otimes|\zeta|^{-1} \in \operatorname{Rep}(M)^{\mathfrak{s}_{M}}$ is an irreducible essentially square-integrable representation with a unitary central character. Hence it is squareintegrable modulo centre and in particular tempered. By Theorem [3.9 $\mathrm{a} \Phi_{M}(\pi \otimes$ $|\zeta|^{-1}$ ) is also tempered.

Let $P_{1}=M_{1} U_{1} \subset G$ be the parabolic subgroup such that $M \supset M_{1} \supset L$ and $\mathrm{d} \Phi_{L}\left(\mathbb{Q} R\left(M_{1}, L\right)\right)$ is spanned by $\mathbb{Q} R(M, L) \cap \mathrm{d} \Phi_{L}^{-1}\left(R^{\vee}\right)$. From (73) we know that $\operatorname{Res}_{\lambda_{M M_{1}}\left(\mathcal{H}^{M_{1}}\right)}^{\mathcal{H}^{M}} \circ \Phi_{M}\left(\pi \otimes|\zeta|^{-1}\right)$ is tempered and nonzero. By Lemma 3.6. a

$$
\Phi_{M_{1}}^{-1} \circ \operatorname{Res}_{\lambda_{M M_{1}}\left(\mathcal{H}^{M_{1}}\right)}^{\mathcal{H}^{M}} \circ \Phi_{M}\left(\pi \otimes|\zeta|^{-1}\right)=J_{P_{1} \cap M}^{M}\left(\pi \otimes|\zeta|^{-1}\right)
$$

is also tempered and nonzero. But [Wal, Lemme III.3.2] says that this contradicts the square-integrability (modulo centre) of $\pi \otimes|\zeta|^{-1}$. (b) This follows from Theorem 
3.9, in the same way as Lemma 3.6.c followed from parts (a) and (b) of that lemma.

(c) This follows from Theorem 3.9. b, Lemma 3.6. c and part (b).

\subsection{Comparison of completions.}

In this paragraph we will show that the equivalences $\Phi_{M}$ induce Morita equivalences between the appropriate Schwartz algebras. In Proposition 1.6 we described the Plancherel isomorphism for the Schwartz completion of an affine Hecke algebra, in terms of the following data:

- the set of parabolic subalgebras $\mathcal{H}^{Q} \rtimes \Gamma_{Q}$ of $\mathcal{H} \rtimes \Gamma$, up to $\Gamma W$-equivalence,

- the tori $T_{\mathrm{un}}^{Q}$,

- the sets $\operatorname{Irr}_{L^{2}}\left(\mathcal{H}^{Q} \rtimes \Gamma_{Q}\right)$, up to the actions of $T_{\mathrm{un}}^{Q}$ and $W \Gamma(Q, Q)$,

- the groupoid $\mathcal{G}$,

- the intertwining operators $I(g, Q, \sigma, t)$ for $g \in \mathcal{G}_{Q, \sigma}$.

These data depend mainly on the categories $\operatorname{Mod}\left(\mathcal{H}^{Q} \rtimes \Gamma_{Q}\right)$. In Condition 3.2 we included the possibility that not the $\mathcal{H}^{M}$, but the $\left(\mathcal{H}^{M}\right)^{\text {op }}$ are affine Hecke algebras, so that $\Phi_{M}$ becomes an equivalence between $\operatorname{Rep}(G)^{\mathfrak{s}}$ and $\operatorname{Mod}\left(\left(\mathcal{H}^{Q} \rtimes \Gamma_{Q}\right)^{\text {op }}\right)$. Then we use Lemma 1.7 to describe the Plancherel isomorphism of $\mathcal{S}(\mathcal{R}, q) \rtimes \Gamma$ in terms of right modules of its subalgebras $\mathcal{H}^{Q} \rtimes \Gamma_{Q}$, that is in terms of the categories $\operatorname{Mod}\left(\mathcal{H}^{M}\right)$. With this in mind, it suffices to consider the case where each $\mathcal{H}^{M}$ is an (extended) affine Hecke algebra.

On the other hand, in Theorem 2.4 the Plancherel isomorphism for $\mathcal{S}(G)^{\mathfrak{s}}$ was formulated in terms of:

- the set of parabolic subgroups $P \supset P_{L}$, up to conjugation by $W_{\mathfrak{s}}$,

- the tori $X_{\text {unr }}(M)$,

- the sets $\operatorname{Irr}_{L^{2}}(M)^{\mathfrak{s}_{M}}$, up to the actions of $X_{\text {unr }}(M)$ and $\operatorname{Stab}_{W_{\mathfrak{s}}}(M)$,

- the groups $W^{\prime}\left(T_{\omega}\right)$,

- the intertwining operators $I\left(w^{\prime}, \omega \otimes \chi\right)$ for $w^{\prime} \in W^{\prime}\left(T_{\omega}\right)$.

We will compare these two data sets, and manipulate them until we get a nice bijection from one side to the other.

By Proposition 3.10, a only the $P$ with $\mathrm{d} \Phi_{L}(\mathbb{Q} R(M, L))$ of the form $\mathbb{Q} Q^{\vee}$ occur in the Plancherel isomorphism, since for the other $P$ the set $\operatorname{Irr}_{L^{2}}(M)^{\mathfrak{s}_{M}}$ is empty. Given $Q \subset \Delta$, we define $\Gamma_{Q}$ as $\Gamma_{M}$, where $\mathbb{Q} R(M, L)=\mathbb{Q} Q^{\vee}$.

By Condition 3.2. iv there is a canonical bijection from the parabolic subgroups $P=M U_{P}$, with $P \supset P_{L}, M \supset L$ and $\mathrm{d} \Phi_{L}(\mathbb{Q} R(M, L))$ of the form $\mathbb{Q} Q^{\vee}$ and modulo conjugation by elements of $W_{\mathfrak{s}}$, to the parabolic subalgebras $\mathcal{H}^{Q} \rtimes \Gamma_{Q}$ of $\mathcal{H}^{G}$, up to association by $W \Gamma$. From Theorem 2.4 one sees that two such Levi subgroups $M \subset G$ are $W_{\mathfrak{s}}$-conjugate if and only if the tempered parts of the two subsets $I_{P}^{G}\left(\operatorname{Rep}(M)^{\mathfrak{s}_{M}}\right)$ coincide. By Condition 3.1, i and Theorem 3.9, a this means precisely that two subsets $\operatorname{ind}_{\left.\lambda_{M G} \mathcal{H}^{G}\right)}^{\mathcal{H}^{M}}\left(\operatorname{Mod}\left(\mathcal{H}^{M}\right)\right)$ of $\operatorname{Mod}\left(\mathcal{H}^{G}\right)$ coincide. By Theorem 1.4 that happens if and only if the two $\mathcal{H}^{M}$ are $\Gamma W$-equivalent. Thus we can pick of representatives for such $P$ modulo $W_{\mathfrak{s}^{-}}$conjugacy, and then the corresponding $\mathcal{H}^{M}$ form representatives for $\Gamma W$-equivalence classes of parabolic subalgebras $\mathcal{H}^{M}=\mathcal{H}^{Q} \rtimes \Gamma_{Q}$ of $\mathcal{H}^{G}$.

By Proposition 3.10, b $\Phi_{M}$ gives a bijection between $\operatorname{Irr}_{L^{2}}(M)^{\mathfrak{s}_{M}}$ and $\operatorname{Irr}_{L^{2}}\left(\mathcal{H}^{M}\right)$. Upon parabolic induction, every $X_{\text {unr }}(M)$-orbit in $\operatorname{Irr}_{L^{2}}(M)^{\mathfrak{s}_{M}}$ (resp. every $T_{\mathrm{un}}^{Q}$ orbit in $\operatorname{Irr}_{L^{2}}\left(\mathcal{H}^{M}\right)$ gives rise to a family of tempered representations in $\operatorname{Rep}(G)^{\mathfrak{s}}$ $\left(\right.$ resp. in $\left.\operatorname{Mod}\left(\mathcal{H}^{G}\right)\right)$. From Theorem 2.4 we see that $I_{P}^{G}(\omega)$ and $I_{P}^{G}\left(\omega^{\prime}\right)$ belong to 
the same such family if and only if $\omega^{\prime}=w(\omega \otimes \chi)$ for some $w \in \operatorname{Stab}_{W_{\mathfrak{s}}}(M)$ and $\chi \in X_{\text {unr }}(M)$. Similarly, by 3.2.ii and Proposition 1.6

$$
\operatorname{ind}_{\lambda_{M G}\left(\mathcal{H}^{M}\right)}^{\mathcal{H}^{G}}(\sigma) \quad \text { and } \quad \operatorname{ind}_{\lambda_{M G}\left(\mathcal{H}^{M}\right)}^{\mathcal{H}^{G}}\left(\sigma^{\prime}\right)
$$

belong to the same family in $\operatorname{Mod}\left(\mathcal{H}^{G}\right)$ if and only if $\sigma^{\prime}=g\left(\sigma \circ \phi_{t}\right)$ for some $g \in \mathcal{G}_{Q Q}$ and $t \in T_{\mathrm{un}}^{Q}$. Applying $\Phi_{G}$ and Condition 3.1.i, we see that the respective equivalence relations on $\operatorname{Irr}_{L^{2}}(M)^{\mathfrak{s}_{M}}$ and $\operatorname{Irr}_{L^{2}}\left(\mathcal{H}^{M}\right)$ agree via $\Phi_{M}$.

Let the set of representatives $(Q, \sigma) / \sim$ be as in (34) Let $(P, M, \omega) / \sim$ be its image under Lemma 3.5 and the $\Phi_{M}^{-1}$. Then $(P, M, \omega) / \sim$ is a set of representatives as in Theorem 2.4. Lemma 3.3.c and Condition 3.1,i guarantee that

$$
\Phi_{G}\left(I_{P}^{G}(\omega \otimes \chi)\right)=\operatorname{ind}_{\left.\lambda_{M G} \mathcal{H}^{M}\right)}^{\mathcal{H}^{G}}\left(\sigma \otimes \Phi_{\mathrm{nr}}(\chi)\right)=\pi\left(Q, \sigma, \Phi_{\mathrm{nr}}(\chi)\right) .
$$

Hence $\Phi_{G}$ matches the finite length tempered elements of $\operatorname{Rep}(G)$ associated to $(P, M, \omega)$ (via Theorem 2.4) with the finite dimensional tempered $\mathcal{H}^{G}$-modules associated to $(Q, \sigma)$ (via Proposition 1.6). By Theorem 2.4 $I_{P}^{G}(\omega \otimes \chi)$ and $I_{P}^{G}\left(\omega \otimes \chi^{\prime}\right)$ are isomorphic if and only $\chi^{\prime}=w^{\prime} \chi$ for some $w^{\prime} \in W^{\prime}\left(T_{\omega}\right)$. Analogously, Proposition 1.6 entails that $\pi(Q, \sigma, t)$ and $\pi\left(Q, \sigma, t^{\prime}\right)$ are isomorphic if and only if $t^{\prime}=g(t)$ for some $g \in \mathcal{G}_{Q, \sigma}$. From this and (62) we see that $\Phi_{\mathrm{nr}}$ (from Lemma 3.3.a) induces a bijection

$$
X_{\mathrm{unr}}(M) / W^{\prime}\left(T_{\omega}\right) \rightarrow T_{\mathrm{un}}^{Q} / \mathcal{G}_{Q, \sigma} \cong X_{\mathrm{unr}}(M) / \mathcal{G}_{Q, \sigma}^{\prime} .
$$

In the proof of Lemma 3.4 we checked that $W_{\mathfrak{s}}$ (resp. $W \Gamma$ ) acts faithfully on $X_{\mathrm{nr}}(L)$ (resp. on $T$ ). Then we see from (56) and (21) that the group actions in (80) are faithful. Comparing the outer sides of (80) and using the same method as in the proof of Lemma 3.4, we deduce that $W^{\prime}\left(T_{\omega}\right)=\mathcal{G}_{Q, \sigma}^{\prime}$ as subgroups of $\operatorname{Aut}\left(X_{\mathrm{unr}}(M)\right)$.

Now we come to the intertwining operators. Recall from (57) and (58) that $I\left(w^{\prime}, \omega \otimes \chi\right)$ for $w^{\prime} \in W^{\prime}\left(T_{\omega}\right)$ comes from a unitary operator

$$
\pi\left(w^{\prime}, \omega, \chi\right): I_{P}^{G}(\omega \otimes \chi) \rightarrow I_{P}^{G}\left(\omega \otimes w^{\prime}(\chi)\right) .
$$

For bookkeeping purposes we replace $T^{Q}$ by $X_{\mathrm{nr}}(M)$ and $\mathcal{G}_{Q, \sigma}$ by $\mathcal{G}_{Q, \sigma}^{\prime}$, at the same time defining

$$
\pi(Q, \sigma, \chi):=\pi\left(Q, \sigma, \Phi_{\mathrm{nr}}(\chi)\right) \quad \text { and } \quad \pi\left(g^{\prime}, Q, \sigma, \chi\right)=\pi\left(g, Q, \sigma, \Phi_{\mathrm{nr}}(\chi)\right)
$$

when $g^{\prime} \in \mathcal{G}_{Q, \sigma}^{\prime}$ is a lift of $g \in \mathcal{G}_{Q, \sigma}$. In particular, for $k \in X_{\mathrm{nr}}(M, \sigma)$ the interwiner $\pi(k, Q, \sigma, \chi)$ is the identity as map on the underlying vector spaces, it only changes $\chi$ to $k \chi$. Then (26) says that the action of $\mathcal{G}_{Q, \sigma}^{\prime}$ in Proposition 1.6 and (34) comes from unitary intertwiners

$$
\pi\left(g^{\prime}, Q, \sigma, \chi\right) \in \operatorname{Hom}_{\mathcal{H}^{G}}\left(\pi(Q, \sigma, \chi), \pi\left(Q, \sigma, g^{\prime}(\chi)\right) .\right.
$$

Lemma 3.11. The intertwining operators (81) and (82) can be normalized so that

$$
\Phi_{G}\left(\pi\left(w^{\prime}, \omega, \chi\right)\right)=\pi\left(g^{\prime}, Q, \sigma, \Phi_{\mathrm{nr}}(\chi)\right)
$$

whenever $w^{\prime}$ corresponds to $g^{\prime}$ under the identification $W^{\prime}\left(T_{\omega}\right)=\mathcal{G}_{Q, \sigma}^{\prime}$ from (80).

Proof. Both (81) and (82) are unique up to scalars, because they depend algebraically on $\chi$ and because for generic $\chi \in X_{\text {unr }}(M)$ the involved representations are irreducible. (The latter follows for example from the Plancherel isomorphisms.) Therefore, if $w^{\prime}=g^{\prime}$ in the indicated way, $\Phi_{G}\left(\pi\left(w^{\prime}, \omega, \chi\right)\right)$ equals $\pi\left(g^{\prime}, Q, \sigma, \Phi_{\mathrm{nr}}(\chi)\right)$ 
up to a complex number of absolute value 1 . To make this scalar 1 , we simply replace $\pi\left(w^{\prime}, \omega, \chi\right)$ by $\Phi_{G}^{-1}\left(\pi\left(g^{\prime}, Q, \sigma, \Phi_{\mathrm{nr}}(\chi)\right)\right)$.

We remark that the normalization from Lemma 3.11 is harmless, because it does not change $I\left(w^{\prime}, \omega \otimes \chi\right)$.

Theorem 3.12. Under the Conditions 3.1, 3.2 and 1.1, $\Phi_{G}: \operatorname{Rep}(G)^{\mathfrak{s}} \rightarrow \operatorname{Mod}\left(\mathcal{H}^{G}\right)$ induces Morita equivalences

$$
\mathcal{S}(G)^{\mathfrak{s}} \sim_{M} \mathcal{S}(\mathcal{R}, q) \rtimes \Gamma \quad \text { and } \quad C_{r}^{*}(G)^{\mathfrak{s}} \sim_{M} C_{r}^{*}(\mathcal{R}, q) \rtimes \Gamma .
$$

Proof. In view of Proposition 1.6 and Theorem 2.4 we have to compare the Schwartz algebras

$$
\begin{aligned}
& \bigoplus_{(P, M, \omega) / \sim}\left(C^{\infty}\left(X_{\mathrm{unr}}(M)\right) \otimes \operatorname{End}_{\mathbb{C}}\left(I_{P}^{G}\left(V_{\omega}\right)^{K_{\mathfrak{s}}}\right)^{W^{\prime}\left(T_{\omega}\right)}\right. \text { and } \\
& \bigoplus_{(Q, \sigma) / \mathcal{G}} C^{\infty}\left(T_{\mathrm{un}}^{Q} ; \operatorname{End}_{\mathbb{C}}\left(V_{Q, \sigma}\right)\right)^{\mathcal{G}_{Q, \sigma}}=\bigoplus_{(Q, \sigma) / \mathcal{G}} C^{\infty}\left(X_{\mathrm{unr}}(M) ; \operatorname{End}_{\mathbb{C}}\left(V_{Q, \sigma}\right)\right)^{\mathcal{G}_{Q, \sigma}^{\prime}} .
\end{aligned}
$$

To justify the equality in the second line, we note that a section of the algebra bundle over $X_{\mathrm{unr}}(M)$ is $X_{\mathrm{nr}}(M, \sigma)$-invariant if and only if it descends to a section of the analogous algebra bundle over $T_{\mathrm{un}}^{Q}$.

By the above constructions the $\Phi_{M}$ provide a bijection between the indexing sets for the sums in (83), so it suffices to compare

$$
\begin{aligned}
& A_{1}:=C^{\infty}\left(X_{\text {unr }}(M) ; \operatorname{End}_{\mathbb{C}}\left(I_{P}^{G}\left(V_{\omega}\right)^{K_{\mathfrak{s}}}\right)\right)^{W^{\prime}\left(T_{\omega}\right)} \text { with } \\
& A_{2}:=C^{\infty}\left(X_{\text {unr }}(M) ; \operatorname{End}_{\mathbb{C}}\left(V_{Q, \sigma}\right)\right)^{\mathcal{G}_{Q, \sigma}^{\prime}}
\end{aligned}
$$

when $(P, M)$ corresponds to $Q$ via Lemma 3.5 and $\Phi_{M}(\omega)=\sigma$. The Morita equivalences $\mathcal{S}\left(G, K_{\mathfrak{s}}\right)^{\mathfrak{s}} \sim_{M} \mathcal{S}(G)^{\mathfrak{s}}$ and $\Phi_{G}$ send $I_{P}^{G}(\omega \otimes \chi)^{K_{\mathfrak{s}}}$ to $\pi(Q, \sigma, \chi)$ and by Lemma 3.11 this is compatible with the intertwining operators. Identifying $W^{\prime}\left(T_{\omega}\right)$ and $\mathcal{G}_{Q, \sigma}^{\prime}$ via (80), we consider the following bimodules for $A_{1}$ and $A_{2}$ :

$$
\begin{aligned}
& B_{1}:=C^{\infty}\left(X_{\mathrm{unr}}(M) ; \operatorname{Hom}_{\mathbb{C}}\left(I_{P}^{G}\left(V_{\omega}\right)^{K_{\mathfrak{s}}}, V_{Q, \sigma}\right)\right)^{W^{\prime}\left(T_{\omega}\right)}, \\
& B_{2}:=C^{\infty}\left(X_{\mathrm{unr}}(M) ; \operatorname{Hom}_{\mathbb{C}}\left(V_{Q, \sigma}, I_{P}^{G}\left(V_{\omega}\right)^{K_{\mathfrak{s}}}\right)\right)^{W^{\prime}\left(T_{\omega}\right)} .
\end{aligned}
$$

Here the $W^{\prime}\left(T_{\omega}\right)$-actions are

$$
\begin{aligned}
& \left(w^{\prime} \cdot f_{1}\right)\left(w^{\prime} \chi\right)=\pi\left(w^{\prime}, \omega \otimes \chi\right) f_{1}(\chi) \pi\left(w^{\prime}, Q, \sigma, \chi\right)^{-1} \quad f_{1} \in B_{1}, \\
& \left(w^{\prime} \cdot f_{2}\right)\left(w^{\prime} \chi\right)=\pi\left(w^{\prime}, Q, \sigma, \chi\right) f_{2}(\chi) \pi\left(w^{\prime}, \omega \otimes \chi\right)^{-1} \quad f_{2} \in B_{2} .
\end{aligned}
$$

Notice that by Lemma 3.11 these are honest group actions, not just up to some scalars. We claim that

$$
B_{1} \otimes_{A_{1}} B_{2} \cong A_{2} \quad \text { and } \quad B_{2} \otimes_{A_{2}} B_{1} \cong A_{1}
$$

as bimodules over $A_{2}$, respectively $A_{1}$. Since all these algebras and modules are of finite rank over $C^{\infty}\left(X_{\mathrm{unr}}(M)\right)^{W^{\prime}\left(T_{\omega}\right)}$, it suffices to check this locally, at any $\chi \in$ $X_{\text {unr }}(M)$. Then the proof of the first half of (85) reduces to checking that

$$
\begin{aligned}
& \operatorname{Hom}_{\mathbb{C}}\left(I_{P}^{G}\left(V_{\omega}\right)^{K_{\mathfrak{s}}}, V_{Q, \sigma}\right)^{W^{\prime}\left(T_{\omega}\right)_{\chi}} \otimes_{\operatorname{End}_{\mathbb{C}}\left(I_{P}^{G}\left(V_{\omega}\right)^{K_{\mathfrak{s}}}\right)^{W^{\prime}\left(T_{\omega}\right)_{\chi}}} \operatorname{Hom}_{\mathbb{C}}\left(V_{Q, \sigma}, I_{P}^{G}\left(V_{\omega}\right)^{K_{\mathfrak{s}}}\right)^{W^{\prime}\left(T_{\omega}\right)_{\chi}} \\
& \cong \operatorname{End}_{\mathbb{C}}\left(V_{Q, \sigma}\right)^{W^{\prime}\left(T_{\omega}\right)_{\chi}},
\end{aligned}
$$

and the other way round for $B_{2} \otimes_{A_{2}} B_{1} \cong A_{1}$. 
By the uniqueness of $\pi\left(w^{\prime}, Q, \sigma, \chi\right)$ up to scalars, $w^{\prime} \mapsto \pi\left(w^{\prime}, Q, \sigma, \chi\right)$ defines a projective representation of $W^{\prime}\left(T_{\omega}\right)_{\chi}$. Let $W^{\prime \prime}$ be a finite central extension of $W^{\prime}\left(T_{\omega}\right)_{\chi}$, such that this lifts to a linear representation of $W^{\prime \prime}$. By (85) the map $w^{\prime} \mapsto \pi\left(w^{\prime}, \omega \otimes \chi\right)$ also lifts to a linear representation of $W^{\prime \prime}$. Then $W^{\prime \prime}$ and $W^{\prime}\left(T_{\omega}\right)$ have the same invariants in the all involved modules, so we can rewrite (86) as

$$
\begin{aligned}
\operatorname{Hom}_{\mathbb{C}\left[W^{\prime \prime}\right]}\left(I_{P}^{G}\left(V_{\omega}\right)^{K_{\mathfrak{s}}}, V_{Q, \sigma}\right) & \otimes_{\operatorname{End}_{\mathbb{C}\left[W^{\prime \prime}\right]}\left(I_{P}^{G}\left(V_{\omega}\right)^{K_{\mathfrak{s}}}\right)} \operatorname{Hom}_{\mathbb{C}\left[W^{\prime \prime}\right]}\left(V_{Q, \sigma}, I_{P}^{G}\left(V_{\omega}\right)^{K_{\mathfrak{s}}}\right) \\
& \cong \operatorname{End}_{\mathbb{C}\left[W^{\prime \prime}\right]}\left(V_{Q, \sigma}\right) .
\end{aligned}
$$

This is a statement about finite dimensional representations of the finite group $W^{\prime \prime}$. One can verfiy (87) by reducing it to the case of irreducible $W^{\prime \prime}$-representations, where it is obvious.

This also proves (86) and (85), and shows that the algebras in (83) are Morita equivalent. Combining that with Theorem 2.4 and (34), we find the desired Morita equivalences of Schwartz algebras.

To prove that $C_{r}(G)^{\mathfrak{s}}$ and $C_{r}(\mathcal{R}, q) \rtimes \Gamma$ are Morita equivalent, we can use exactly the same argument. We only have to replace $C^{\infty}$ by continuous functions everywhere, and to use Theorem 2.5 instead of Theorem 2.4.

\section{Hecke algebras from Bushnell-Kutzko types}

Let $L \subset G$ be a Levi subgroup and let $\sigma \in \operatorname{Irr}(L)$ be supercuspidal. Recall from $[\mathrm{BuKu}, \S 4]$ that a type for $\mathfrak{s}=[L, \sigma]_{G}$ consists of a compact open subgroup $J \subset G$, and a $\lambda \in \operatorname{Irr}(J)$, such that $\operatorname{Rep}(G)^{\mathfrak{s}}$ is precisely the category of smooth $G$-representations which are generated by their $\lambda$-isotypical subspace. To such a type one associates the algebra

$$
\mathcal{H}(G, J, \lambda)=\operatorname{End}_{G}\left(\operatorname{ind}_{J}^{G} \lambda\right)
$$

which (by definition) acts from the right on $\operatorname{ind}_{J}^{G} \lambda$. Then there is a Morita equivalence

$$
\begin{array}{ccc}
\Phi_{G}: \operatorname{Rep}(G)^{\mathfrak{s}} & \rightarrow & \operatorname{Mod}(\mathcal{H}(G, J, \lambda)) \\
\pi & \mapsto \operatorname{Hom}_{J}(\lambda, \pi) \cong \operatorname{Hom}_{G}\left(\operatorname{ind}_{J}^{G} \lambda, \pi\right) .
\end{array}
$$

For a Levi subgroup $M \subset G$ containing $L$, Bushnell and Kutzko [BuKu, §8] developed the notion that $(J, \lambda)$ covers a $[L, \sigma]_{M}$-type $\left(J_{M}, \lambda_{M}\right)$. Roughly speaking, this means that $J_{M}=J \cap M$, that $\lambda_{M}=\operatorname{Res}_{J_{M}}^{J} \lambda$ and that $\mathcal{H}(G, J, \lambda)$ contains invertible "strongly positive" elements. Under these conditions, writing $\mathfrak{s}_{M}=[L, \sigma]_{M}$, there is a Morita equivalence $\Phi_{M}: \operatorname{Rep}(M)^{\mathfrak{s}_{M}} \rightarrow \operatorname{Mod}\left(\mathcal{H}\left(M, J_{M}, \lambda_{M}\right)\right)$ as in (88), which is in several ways compatible with $\Phi_{G}$.

Lemma 4.1. Suppose that $(J, \lambda)$ is a cover of a $[L, \sigma]_{L}$-type $\left(J_{L}, \lambda_{L}\right)$. Then Condition 3.1 is fulfilled, with $\left.\mathcal{H}^{M}=\mathcal{H}\left(M, J_{M}, \lambda_{M}\right)\right)$.

Proof. Let $P$ and $P^{\prime}$ be as in the condition. By [BuKu, Proposition 8.5] $\left(J_{M^{\prime}}, \lambda_{M^{\prime}}\right)$ is a $\mathfrak{s}_{M^{\prime}}$-type, $\left(J_{M}, \lambda_{M}\right)$ is a $\mathfrak{s}_{M}$-type and the former covers the latter.

By $[\mathrm{BuKu}$, Corollary 8.4] there exists a unique algebra monomorphism

$$
t_{\bar{P} \cap M}: \mathcal{H}\left(M, J_{M}, \lambda_{M}\right) \rightarrow \mathcal{H}\left(M^{\prime}, J_{M^{\prime}}, \lambda_{M^{\prime}}\right)
$$

such that

$$
\operatorname{Res}_{t_{\bar{P} \cap M}\left(\mathcal{H}\left(M, M_{M}, \lambda_{M}\right)\right)}^{\mathcal{H}\left(M^{\prime}, J_{M^{\prime}, \lambda_{M^{\prime}}}\right)} \circ \Phi_{M^{\prime}}=\Phi_{M} \circ \operatorname{pr}_{\mathfrak{s}_{M}} \circ R R_{\bar{P} \cap M^{\prime}}^{M^{\prime}}
$$


Here $R_{\bar{P} \cap M^{\prime}}^{M^{\prime}}$ means the unnormalized parabolic restriction functor. To obtain the version with the normalized Jacquet functor $J M^{M^{\prime}} \cap M^{\prime}$, we must adjust $t_{\bar{P} \cap M}$ by the square root of a modular character. This yields our $\lambda_{M M^{\prime}}$. The uniqueness of $\lambda_{M M^{\prime}}$ and the transitivity of normalized Jacquet restriction entail that

$$
\lambda_{M^{\prime} M^{\prime \prime}} \circ \lambda_{M M^{\prime}}=\lambda_{M M^{\prime \prime}} \text { when } P \subset P^{\prime} \subset P^{\prime \prime} \subset G \text {. }
$$

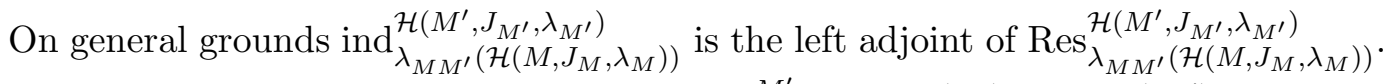
By Bernstein's second adjointness theorem $I_{P \cap M^{\prime}}^{M^{\prime}}: \operatorname{Rep}(M) \rightarrow \operatorname{Rep}\left(M^{\prime}\right)$ is the left adjoint of $J_{\bar{P} \cap M^{\prime}}^{M^{\prime}}$. Hence

$$
I_{P \cap M^{\prime}}^{M^{\prime}}: \operatorname{Rep}(M)^{\mathfrak{s}_{M}} \rightarrow \operatorname{Rep}\left(M^{\prime}\right)^{\mathfrak{s}_{M^{\prime}}}
$$

is the left adjoint of $\operatorname{pr}_{\mathfrak{s}_{M}} \circ J \frac{M^{\prime}}{P \cap M^{\prime}}$. By the uniqueness of adjoints

$$
\Phi_{M^{\prime}} \circ I_{P \cap M^{\prime}}^{M^{\prime}}=\operatorname{ind}_{\lambda_{M M^{\prime}}\left(\mathcal{H}\left(M, J_{M}, \lambda_{M}\right)\right)}^{\mathcal{H}\left(M^{\prime}, J_{M^{\prime}}, \lambda_{M^{\prime}}\right)} \circ \Phi_{M} .
$$

Having checked Condition 3.1 in a general framework, we turn to more specific instances where Condition 3.2 holds. In most cases the intermediate algebras $\mathcal{H}^{M}$ are not mentioned explicitly in the literature. One can obtain them by applying the same references to the group $M$ instead of $G$. Using the canonical construction of $\lambda_{M M^{\prime}}$ as in the proof of Lemma 4.1, Condition 3.2, ii will be satisfied automatically in those cases. We will check the remaining conditions, mainly by providing relevant references. Recall that to achieve Condition 3.2. iii we can use the method described on page 28 .

\section{Iwahori-spherical representations.}

This is the classical case. Let $\mathfrak{o}_{F}$ be the ring of integers of the non-archimedean local field $F$, let $\mathfrak{p}_{F}$ be its maximal ideal, and let $k_{F}=\mathfrak{o}_{F} / \mathfrak{p}_{F}$ be the residue field. Choose an apartment $\mathbb{A}$ of the Bruhat-Tits building of $G$ and let $L$ be the correponding minimal $F$-Levi subgroup of $G$. Let $I$ be an Iwahori subgroup of $G$ associated to a chamber of $\mathbb{A}$. Let $P_{L}$ be the parabolic subgroup of $G$ with Levi factor $L$, such that the reduction of $I$ modulo $\mathfrak{p}_{F}$ is $P_{L}\left(k_{F}\right)$.

Borel [Bor] showed that the trivial representation of $I$ is a $\mathfrak{s}$-type, where $\mathfrak{s}=$ $\left[L, \operatorname{triv}_{L}\right]_{G}$. Borel assumes that $G$ is semisimple, but it is easy to generalize his arguments to reductive $G$.

By [IwMa, $\S 3]$ there is a *algebra isomorphism

$$
C_{c}(I \backslash G / I) \cong \mathcal{H}(G, I, \text { triv }) \cong \mathcal{H}\left(X_{*}(L), R^{\vee}(G, L), X^{*}(L), R(G, L), \Delta, q_{I}\right),
$$

where the basis $\Delta$ is determined by $P_{L}$ and $q_{I, \alpha}=\operatorname{vol}\left(I s_{\alpha} I\right) / \operatorname{vol}(I)$ for a simple reflection $s_{\alpha}$. From [Bor, §3.1] one sees that Conditions 3.2. iii and iv hold. Here $\Gamma_{M}=1$ for all $M$, so Condition 1.1 is vacuous.

Of course Theorem 3.9 was already known for irreducible Iwahori-spherical representations. Indeed, by [KaLu, Section 8] and [ABPS1, Theorem 15.1.(2) and Proposition 16.6] the bijection $\operatorname{Irr}(G)^{\mathfrak{s}} \rightarrow \operatorname{Irr}(\mathcal{H}(G, I$, triv $))$ preserves temperedness and essential square-integrability. Moreover Theorem 3.12 has been proven for Schwartz algebras in [DeOp, Theorem 10.2]: (89) extends to an isomorphism of Fréchet *algebras

$$
\mathcal{S}(I \backslash G / I) \cong \mathcal{S}\left(X_{*}(L), R^{\vee}(G, L), X^{*}(L), R(G, L), \Delta, q_{I}\right) .
$$


Principal series representations of split groups.

Suppose that $G$ is $F$-split and let $T$ be a maximal split torus of $G$. Fix a smooth character $\chi_{\mathfrak{s}} \in \operatorname{Irr}(T)$ and put $\mathfrak{s}=\left[T, \chi_{\mathfrak{s}}\right]_{G}$, so that

$$
X_{\mathrm{nr}}(T) \rightarrow T_{\mathfrak{s}}: \chi \mapsto \chi \chi_{\mathfrak{s}}
$$

is a homeomorphism. Notice that $\chi$ restricted to the unique maximal compact subgroup $T_{\text {cpt }}$ of $T$ is a type for $\left[T, \chi_{\mathfrak{s}}\right]_{T}$. By [Roc1, Lemma 6.2] there exist a root subsystem $R_{\mathfrak{s}} \subset R^{\vee}(G, T)$ and a subgroup $\mathfrak{R}_{\mathfrak{s}} \subset W_{\mathfrak{s}}$ such that $W_{\mathfrak{s}}=W\left(R_{\mathfrak{s}}\right) \rtimes \mathfrak{R}_{\mathfrak{s}}$.

Theorem 4.2. [Roc1, Theorem 6.3]

There exists a type $(J, \lambda)$ for $\mathfrak{s}$ and a *algebra isomorphism

$$
\mathcal{H}(G, J, \lambda) \cong \mathcal{H}\left(T_{\mathfrak{s}}, R_{\mathfrak{s}}, q\right) \rtimes \mathfrak{R}_{\mathfrak{s}},
$$

where $q_{\alpha}=\left|k_{F}\right|$ for all $\alpha \in R_{\mathfrak{s}}$. Moreover $(J, \lambda)$ is a cover of $\left(T_{\mathrm{cpt}}, \chi\right)$.

Furthermore Conditions 3.2, iii and iv hold by construction. If $\mathbb{Q} R(M, T)=\mathbb{Q} Q^{\vee}$, then $X \rtimes W_{Q} \Gamma_{Q} \subset X_{*}(T) \rtimes W(M, T)$, so by Remark 1.2 Condition 1.1 holds as well.

We note that for these Bernstein components Theorem 3.9.b was already proven in [Roc1, Theorem 10.7], while Theorem 3.9] a follows from [DeOp, Theorem 10.1], using [Roc1, Section 8].

\section{Level zero representations.}

These are $G$-representations which are generated by non-zero vectors fixed by the pro-unipotent radical of a parahoric subgroup of $G$. Iwahori-spherical representations constitute the most basic example of this kind. A type $(J, \lambda)$ for any Bernstein component $\mathfrak{s}$ consisting of level zero representations was exhibited in [Mor1], while it was proven in [Mor2, Theorem 4.9] that it actually is a type. More precisely, by [Mor2, $\S 3.8](J, \lambda)$ is a cover of a type for the underlying supercuspidal Bernstein component of a Levi subgroup $L$ of $G$.

By [Mor1, Theorem 7.12] (see also [Lus2])

$$
\mathcal{H}(G, J, \lambda) \cong \mathcal{H}(\mathcal{R}, q) \rtimes \mathbb{C}\left[\Gamma, \bigsqcup_{\mathfrak{s}}\right]
$$

for suitable $\mathcal{R}, q$ and $\Gamma$. In all examples of level zero Bernstein blocks which have been worked out, the 2-cocycle $\hbar_{\mathfrak{s}}$ of $\Gamma$ is trivial. But even if it were non-trivial, we could easily deal with it. There always exists a finite central extension

$$
1 \rightarrow \Gamma_{1} \rightarrow \Gamma_{2} \stackrel{\phi_{\Gamma}}{\longrightarrow} \Gamma \rightarrow 1
$$

such that the pullback of $\mathfrak{h}_{\mathfrak{s}}$ to $\Gamma_{2}$ splits. Then $\mathcal{H}(G, J, \lambda)$ can be regarded as the direct summand of $\mathcal{H}(\mathcal{R}, q) \rtimes \Gamma_{2}$ associated to a minimal central idempotent $p_{\natural_{\mathfrak{s}}} \in$ $\mathbb{C}\left[\Gamma_{1}\right]$. The algebra $\mathcal{H}(\mathcal{R}, q) \rtimes \Gamma_{2}$, with the parabolic subalgebras $\mathcal{H}^{Q} \rtimes \phi_{\Gamma}^{-1}\left(\Gamma_{Q}\right)$, is of the kind studied in Section 1. In this situation the Conditions 3.1 and 3.2 must be adjusted slightly, now each $\mathcal{H}^{M}$ should be $p_{\natural_{\mathfrak{s}}} \mathcal{H}^{Q} \rtimes \phi_{\Gamma}^{-1}\left(\Gamma_{M}\right)$ for some $Q \subset \Delta$. With these minor modifications, all the arguments in Section 3 remain valid.

Conditions 3.2, iii and iv follow from the setup in [Mor1, §3.12-3.14] and [Mor2, $\S 1.10$ ], combined with the description of $\mathcal{R}$ in [Mor1, Proposition 7.3]. The groups $\Gamma_{Q}$ for $\mathbb{Q} Q^{\vee}=\mathbb{Q} R(M, L)$ satisfy Condition 1.1 because they are contained in $X \rtimes$ $W(M, S)$, where $W(M, S)$ is the Weyl group of $M$ with respect to a maximal $F$-split torus $S \subset L$.

As in the above examples, there is previous work on temperedness also. It is claimed in [DeOp, Theorem 10.1] that Theorem 3.9, a holds here. For this one needs 
to know that (90) preserves the traces (maybe up to a positive factor) and the natural *-operations. The former follows from the support of the basis elements $T_{w}$ of $\mathcal{H}(G, J, \lambda)$ constructed in [Mor1] (only the unit element $T_{e}$ is supported on $J$ ). For a simple (affine) reflection $s$, both $T_{s}$ and $T_{s}^{*}$ have support $J s J$, so they differ only by a scalar factor. They also satisfy the same quadratic relation, so $T_{s}^{*}=T_{s}$. This implies that (90) is an isomorphism of *-algebras.

Knowing that DeOp, Theorem 10.1] applies, and together with [ABPS1, Lemma 16.5] it also gives Theorem 3.9.b.

Inner forms of $\mathrm{GL}_{n}(F)$.

Let $D$ be a division algebra with centre $F$. Every Levi subgroup of $G=\mathrm{GL}_{m}(D)$ is of the form $L=\prod_{i} \mathrm{GL}_{m_{i}}(D)^{e_{i}}$, where $\sum_{i} m_{i} e_{i}=m$. Fix a supercuspidal $\omega \in$ $\operatorname{Irr}(L)$, of the form $\omega=\bigotimes_{i=1}^{k} \omega_{i}^{\otimes e_{i}}$, where $\omega_{i} \in \operatorname{Irr}\left(\mathrm{GL}_{m_{i}}(D)\right)$ is supercuspidal and not inertially equivalent with $\omega_{j}$ if $i \neq j$. Then $T_{\mathfrak{s}} \cong \prod_{i=1}^{k}\left(\mathbb{C}^{\times}\right)^{e_{i}}, R_{\mathfrak{s}}$ is of type $\prod_{i=1}^{k} A_{e_{i}-1}$ and the stabilizer of $\mathfrak{s}=[\omega, L]_{G}$ in $W(G, L)$ is $W\left(R_{\mathfrak{s}}\right) \cong \prod_{i=1}^{k} S_{e_{i}}$.

Theorem 4.3. Séc, SéSt

There exists a type $(J, \lambda)$ for $\mathfrak{s}$, which is a cover of a $[\omega, L]_{L}$-type. There exists a parameter function $q_{\mathfrak{s}}: R_{\mathfrak{s}} \rightarrow q^{\mathbb{N}}$ such that there is an isomorphism of ${ }^{*}$-algebras

$$
\mathcal{H}(G, J, \lambda) \cong \mathcal{H}\left(X^{*}\left(T_{\mathfrak{s}}\right), R_{\mathfrak{s}}, X_{*}\left(T_{\mathfrak{s}}\right), R_{\mathfrak{s}}^{\vee}, q_{\mathfrak{s}}\right),
$$

where the right hand side is a tensor product of affine Hecke algebras of type $\mathrm{GL}_{e}$ with $e \leq m$. Moreover this isomorphism sends the natural trace of $\mathcal{H}(G, J, \lambda)$ to a positive multiple of the trace of the right hand side.

We remark that the claims about the ${ }^{*}$ and the traces are not made explicit in Séc, SéSt]. They can be deduced in the same way as for level zero representations, see above. With [DeOp, Theorem 10.1] that proves Proposition 3.10 for these groups.

Via the tensor product factorization Condition 3.2, iii reduces to the case of a supercuspidal representation $\sigma^{\otimes e}$ of $\mathrm{GL}_{r}(D)^{e}$. There it is a consequence of the constructions involved in [Séc, Théorème 4.6], which entail that the same notion of positivity in real tori is used for $\left(\mathrm{GL}_{r}(D)^{e}, \mathrm{GL}_{1}(D)^{r e}\right)$ and for $\mathcal{H}\left(\mathrm{GL}_{e}, q\right)$. Condition 3.2. iv is irrelevant because all the groups $\Gamma_{M}$ are trivial.

For the Schwartz algebras of these groups Theorem 3.12 can be found in ABPS2, Theorem 6.2]. The proof over there is similar but simpler, because not all complications from Section 3 arise.

Inner forms of $\mathrm{SL}_{n}(F)$.

Let $G$ be the kernel of the reduced norm map $\operatorname{GL}_{m}(D) \rightarrow F^{\times}$. It is an inner form of $\mathrm{SL}_{n}(F)$, and every inner form looks like this. It was shown in ABPS1 that for every inertial equivalence class $\mathfrak{s}, \mathcal{H}(G)^{\mathfrak{s}}$ is Morita equivalent with an algebra which is closely related to affine Hecke algebras of type $\mathrm{GL}_{e}$ (yet is of a more general kind). It is not known whether there exists a $\mathfrak{s}$-type for every $\mathfrak{s}$, but in any case the constructions in ABPS1 are derived from the work of Sécherre and Stevens on inner forms of $\mathrm{GL}_{n}(F)$, so types are not far away. Condition 3.1.i is ABPS2, Theorem 1.5.b], the maps $\lambda_{M M^{\prime}}$ are simply inclusions, and Condition [3.1,ii follows from that by the uniqueness of adjoints.

Condition 3.2 does not hold precisely for the algebras $\mathcal{H}^{M}$ obtained in this setting (in fact the Plancherel isomorphism for these $\mathcal{H}^{M}$ has not been worked out), so we 
cannot apply Proposition 3.10 or Theorem 3.12, Nevertheless the conclusions of these results hold, see [ABPS2].

Let us summarize the conclusions from this section.

Corollary 4.4. Let $\mathfrak{s}=[L, \sigma]_{G}$ be an inertial equivalence class of the kind discussed in this paragraph (principal series of split group, level zero, inner form of $\mathrm{GL}_{n}(F)$ or $\mathrm{SL}_{n}(F)$ ). Then $\mathcal{S}(G)^{\mathfrak{s}}$ is Morita equivalent to the Schwartz completion of an extended affine Hecke algebra and $C_{r}^{*}(G)^{\mathfrak{s}}$ is Morita equivalent to the $C^{*}$-completion of the same extended affine Hecke algebra.

Proof. Except for the last case, this follows by applying Theorem 3.12 . We just checked that all its assumptions are fulfilled. For the inner forms of $\mathrm{SL}_{n}(F)$, ABPS2, Theorem 6.4] gives the result in the case of Schwartz algebras. Like the proof of Theorem [3.12, the method in ABPS2, §6.2] also works for the $C^{*}$-algebras, with minor modifications.

\section{Hecke Algebras from Bernstein's Progenerators}

We return to the notations from Sections 2 and 3. Let $\mathfrak{s}=[L, \sigma]_{G}$ be any inertial equivalence class for $G$. Bernstein $\mathrm{BeRu}, \S 3]$ constructed a projective generator $\Pi_{\mathfrak{s}}$ for the category $\operatorname{Rep}(G)^{\mathfrak{s}}$. By [Ren, VI.10.1], for any Levi subgroup $M \subset G$ containing $L$ :

$$
\Pi_{\mathfrak{s}_{M}}=I_{P_{L} \cap M}^{M}\left(\Pi_{\mathfrak{s}_{L}}\right)
$$

and this is a progenerator of $\operatorname{Rep}(M)^{\mathfrak{s}_{M}}$. In other words, the map

$$
\Phi_{M}: V \mapsto \operatorname{Hom}_{M}\left(I_{P_{L} \cap M}^{M}\left(\Pi_{\mathfrak{s}_{L}}\right), V\right)
$$

is an equivalence between $\operatorname{Rep}(M)^{\mathfrak{s}_{M}}$ and the category of right modules of $\operatorname{End}_{M}\left(I_{P_{L} \cap M}^{M} \Pi_{\mathfrak{s}_{L}}\right)$. For $P_{L} \subset P=M U_{P} \subset G$ we put

$$
\mathcal{H}^{M}=\operatorname{End}_{M}\left(I_{P_{L} \cap M}^{M} \Pi_{\mathfrak{s}_{L}}\right)^{\text {op }}=\operatorname{End}_{M}\left(\Pi_{\mathfrak{s}_{M}}\right)^{\text {op }} .
$$

Then $\Phi_{M}$ provides an equivalence of categories $\operatorname{Rep}(M)^{\mathfrak{s}_{M}} \rightarrow \operatorname{Mod}\left(\mathcal{H}^{M}\right)$.

Lemma 5.1. In the above setting Condition 3.1 is fulfilled.

Proof. The functoriality of normalized parabolic induction gives natural injections

$$
\lambda_{M M^{\prime}}: \mathcal{H}^{M} \rightarrow \mathcal{H}^{M^{\prime}} \text { for } \quad P \subset P^{\prime} \subset G .
$$

By naturality the $\lambda_{M M^{\prime}}$ satisfy Condition 3.1,iii. By Bernstein's second adjointness theorem, for $V^{\prime} \in \operatorname{Rep}\left(M^{\prime}\right)^{\mathfrak{s} M^{\prime}}$ :

$$
\begin{aligned}
& \Phi_{M}\left(J \frac{M^{\prime}}{\bar{P} \cap M^{\prime}} V^{\prime}\right)=\operatorname{Hom}_{M}\left(I_{P_{L} \cap M}^{M} \Pi_{\mathfrak{s}_{L}}, J \frac{M^{\prime}}{\bar{P} \cap M^{\prime}} V^{\prime}\right) \\
& \cong \operatorname{Hom}_{M^{\prime}}\left(I_{P \cap M^{\prime}}^{M^{\prime}} I_{P_{L} \cap M}^{M} \Pi_{\mathfrak{s}_{L}}, V^{\prime}\right) \\
& \cong \operatorname{Hom}_{M^{\prime}}\left(I_{P_{L} \cap M^{\prime}}^{M^{\prime}} \Pi_{\mathfrak{s}_{L}}, V^{\prime}\right)=\Phi_{M^{\prime}}\left(V^{\prime}\right)
\end{aligned}
$$

as $\mathcal{H}^{M}$-modules (via $\lambda_{M M^{\prime}}$ ). This establishes the first commutative diagram in Condition 3.1. As in the proof of Lemma 4.1, the second commutative diagram follows from that by invoking the uniqueness of left adjoints.

In the remainder of this section we assume that $G$ is: 
- either a symplectic group, not necessarily split,

- or a special orthogonal group, not necessarily split,

- or an inner form of $\mathrm{GL}_{n}(F)$.

Besides the discussion of inner forms of $\mathrm{GL}_{n}(F)$ in the previous section, we point out that types for Bernstein components of symplectic or special orthogonal groups have been constructed in [MiSt]. However, as far as we know the Hecke algebras associated to these types are in only few cases known explicitly.

For the groups listed above, Heiermann has subjected $\left(\mathcal{H}^{G}\right)^{\text {op }}=\operatorname{End}_{G}\left(I_{P_{L}}^{G} \Pi_{\mathfrak{s}_{L}}\right)$ to a deep study. In Hei1 he proved that it is an extended affine Hecke agebra with positive parameters. The constructions in [Hei1, §5] are such that every $\operatorname{End}_{M}\left(I_{P_{L} \cap M}^{M} \Pi_{\mathfrak{s}_{L}}\right)$ arises as a parabolic subalgebra. For Condition 3.2, iii see [Hei2, §3]. It served as a step towards Theorem 3.9 for these groups [Hei2, Théorème 5].

By [Hei1, Proposition 1.15] the groups $W_{Q} \Gamma_{Q}$ are always contained in $W\left(\tilde{R}_{Q}\right)$ where $\tilde{R}_{Q} \subset \mathbb{Q} R_{Q}$ is a larger root system. In view of Remark 1.2, Condition 3.2.iv holds.

In fact, a more precise description of the root data and the groups $\Gamma_{M}$ is available. By [Hei1, 1.13] the root datum underlying the affine Hecke algebra $\operatorname{End}_{G}\left(I_{P_{L}}^{G} \Pi_{\mathfrak{s}_{L}}\right)$ is a tensor product of root data of four types: $\mathrm{GL}_{n}, \mathrm{Sp}_{2 n}, \mathrm{SO}_{2 n+1}$ and $\mathrm{SO}_{2 n}$. The groups $\Gamma_{M}$ are described in [Hei1, 1.15], but unfortunately some elements were overlooked, for the complete picture we refer to [Gol]. The only nontrivial $\Gamma_{M}$ come from the type $D$ factors, it can happen that for a root datum of type $\left(\mathrm{SO}_{2 n}\right)^{e}$ we have (extended) Weyl groups

$$
W_{M} \cong W\left(D_{n}\right)^{e}, \quad W_{M} \Gamma_{M} \cong W\left(D_{n e}\right) \cap W\left(B_{n}\right)^{e} .
$$

Then $\left|\Gamma_{M}\right|=2^{e-1}$. In the above setting, Theorem 3.12 says:

Theorem 5.2. Let $G$ be a symplectic group or a special orthogonal group over $F$ (not necessarily split), or an inner form of $G L_{n}(F)$. Let $\mathfrak{s}$ be any inertial equivalence class for $G$.

Then $\mathcal{S}(G)^{\mathfrak{s}}$ is Morita equivalent with the Schwartz completion of an extended affine Hecke algebra. The underlying root datum is a tensor product of root data of type $G L_{n}, S p_{2 n}, S O_{2 n+1}$ and $S O_{2 n}$, and the group $\Gamma$ is a direct product of groups $\Gamma_{M}$ as in (91). Furthermore $C_{r}^{*}(G)^{\mathfrak{s}}$ is Morita equivalent with the $C^{*}$-completion of that extended affine Hecke algebra.

Theorem 5.2 was one of the motivations for the author to write a paper about the $\mathrm{K}$-theory of $C^{*}$-completions of (extended) affine Hecke algebras [Sol5]. It enables us to show that the K-groups of the reduced $C^{*}$-algebras of the above groups are torsion-free.

Theorem 5.3. Let $G$ be as in Theorem 5.2. Then $K_{*}\left(C_{r}^{*}(G)\right)$ is a free abelian group. It is countably infinite (unless $G=1$ ).

Proof. Recall the Bernstein decomposition from (45):

$$
C_{r}^{*}(G) \cong \prod_{\mathfrak{s} \in \mathfrak{B}(G)} C_{r}^{*}(G)^{\mathfrak{s}} .
$$

Since topological K-theory is a continuous functor on the category of Banach algebras, it commutes with direct sums. This reduces the theorem to one factor $C_{r}^{*}(G)^{\mathfrak{s}}$. By Morita invariance and Theorem [5.2, it suffices to show that the K-theory of the $C^{*}$-completion of an extended affine Hecke algebra as in Theorem 5.2 is a finitely 
generated free abelian group. It was checked in [Sol5, (62)] that the Künneth theorem for topological K-theory $[\mathrm{Sch}]$ applies to such algebras. Thus we only need to prove the result when the underlying root datum is of type $\mathrm{GL}_{n}, \mathrm{Sp}_{2 n}$ or $\mathrm{SO}_{2 n+1}$ and $\Gamma$ is trivial, and when the root datum is of type $\left(\mathrm{SO}_{2 n}\right)^{e}$ and $\Gamma$ is as in (911). These K-groups were computed in [Sol5], see respectively Theorem 3.2, Theorem 3.3, (128), and Proposition 3.5. They are free abelian and have finite rank.

\section{REFERENCES}

[ABPS1] A.-M. Aubert, P.F. Baum, R.J. Plymen, M. Solleveld, "Hecke algebras for inner forms of p-adic special linear groups", J. Inst. Math. Jussieu 16.2 (2017), 351-419.

[ABPS2] A.-M. Aubert, P.F. Baum, R.J. Plymen, M. Solleveld, "The noncommutative geometry of inner forms of $p$-adic special linear groups", arXiv:1505.04361, 2015.

[BaCi] D. Barbasch, D. Ciubotaru, "Unitary equivalences for reductive $p$-adic groups", Amer. J. Math. 135.6 (2013), 1633-1674.

[BaMo] D. Barbasch, A. Moy, "A unitarity criterion for p-adic groups" Inv. Math. 98 (1989), 19-37.

[BeDe] J. Bernstein, P. Deligne, "Le "centre" de Bernstein", pp. 1-32 in: Représentations des groupes réductifs sur un corps local, Travaux en cours, Hermann, Paris, 1984.

[BeRu] J. Bernstein, K.E. Rumelhart, "Representations of p-adic groups", draft, 1993.

[Bor] A. Borel, "Admissible representations of a semi-simple group over a local field with vectors fixed under an Iwahori subgroup", Inv. Math. 35 (1976), 233-259.

[BHK] C.J. Bushnell, G. Henniart, P.C. Kutzko, "Types and explicit Plancherel formulae for reductive p-adic groups", pp. 55-80 in: On certain L-functions, Clay Math. Proc. 13, American Mathematical Society, 2011.

[BuKu] C.J. Bushnell, P.C. Kutzko, "Smooth representations of reductive $p$-adic groups: structure theory via types", Proc. London Math. Soc. 77.3 (1998), 582-634.

[Cas] W. Casselman, "Introduction to the theory of admissible representations of $p$-adic reductive groups", preprint, 1995.

[Ciu] D. Ciubotaru, "Types and unitary representations of reductive $p$-adic groups", arXiv:1707.06506, 2017.

[DeOp] P. Delorme, E.M. Opdam, "The Schwartz algebra of an affine Hecke algebra", J. reine angew. Math. 625 (2008), 59-114.

[Gol] D. Goldberg, "Reducibility of induced representations for $\mathrm{Sp}(2 \mathrm{~N})$ and $\mathrm{SO}(\mathrm{N})$ ", Amer. J. Math. 116.5 (1994), 1101-1151.

[HC] Harish-Chandra, "The Plancherel formula for reductive $p$-adic groups", Collected papers Vol. IV, 353-367, Springer-Verlag, New York, 1984.

[Hei1] V. Heiermann, "Opérateurs d'entrelacement et algèbres de Hecke avec paramètres d'un groupe réductif $p$-adique - le cas des groupes classiques", Selecta Math. 17.3 (2011), 713-756.

[Hei2] V. Heiermann, "Algèbres de Hecke avec paramètres et représentations d'un groupe $p$-adique classique: préservation du spectre tempéré", J. Algebra 371 (2012), 596-608.

[IwMa] N. Iwahori, H. Matsumoto, "On some Bruhat decomposition and the structure of the Hecke rings of the p-adic Chevalley groups", Inst. Hautes Études Sci. Publ. Math 25 (1965), 5-48.

[Kaz] D. Kazhdan, "Cuspidal geometry of p-adic groups", J. Analyse Math. 47 (1986), 1-36.

[KaLu] D. Kazhdan, G. Lusztig, "Proof of the Deligne-Langlands conjecture for Hecke algebras", Invent. Math. 87 (1987), 153-215.

[Lus1] G. Lusztig, "Affine Hecke algebras and their graded version", J. Amer. Math. Soc. 2.3 (1989), 599-635.

[Lus2] G. Lusztig, "Classification of unipotent representations of simple p-adic groups", Int. Math. Res. Notices 11 (1995), 517-589.

[MiSt] M. Miyauchi, S. Stevens, "Semisimple types for $p$-adic classical groups", Math. Ann. 358 (2014), 257-288.

[Mor1] L. Morris, "Tamely ramified intertwining algebras", Invent. Math. 114.1 (1993), 1-54.

[Mor2] L. Morris, "Level zero G-types", Compositio Math. 118.2 (1999), 135-157.

[Opd] E.M. Opdam, "On the spectral decomposition of affine Hecke algebras", J. Inst. Math. Jussieu 3.4 (2004), 531-648. 
[Ply] R.J. Plymen, "Reduced $C^{*}$-algebra for reductive $p$-adic groups", J. Funct. Anal. 88.2 (1990), 251-266.

[RaRa] A. Ram, J. Ramagge, "Affine Hecke algebras, cyclotomic Hecke algebras and Clifford theory", pp. 428-466 in: A tribute to C.S. Seshadri (Chennai 2002), Trends in Mathematics, Birkhäuser, 2003.

[Ren] D. Renard, Représentations des groupes réductifs p-adiques, Cours spécialisés 17, Société Mathématique de France, 2010.

[Roc1] A. Roche, "Types and Hecke algebras for principal series representations of split reductive p-adic groups", Ann. Sci. École Norm. Sup. 31.3 (1998), 361-413.

[Roc2] A. Roche, "Parabolic induction and the Bernstein decomposition", Comp. Math. 134 (2002), $113-133$.

[Sau] F. Sauvageot, "Principe de densité pour les groupes réductifs", Comp. Math. 108 (1997), 151-184.

[SSZ] P. Schneider, E.-W. Zink, "K-types for the tempered components of a $p$-adic general linear group. With an appendix by Schneider and U. Stuhler", J. reine angew. Math. 517 (1999), 161-208.

[ScZi] P. Schneider, E.-W. Zink, "The algebraic theory of tempered representations of $p$-adic groups. Part II: Projective generators", Geom. Func. Anal. 17.6 (2008), 2018-2065.

[Sch] C. Schochet, "Topological methods for $C^{*}$-algebras. II. Geometry resolutions and the Künneth formula", Pacific J. Math. 98.2 (1982), 443-458.

[Séc] V. Sécherre, "Représentations lisses de $G L_{m}(D)$ III: types simples", Ann. Scient. Éc. Norm. Sup. 38 (2005), 951-977.

[SéSt] V. Sécherre, S. Stevens, "Smooth representations of $G L(m, D)$ VI: semisimple types", Int. Math. Res. Notices 13 (2012), 2994-3039.

[Sol1] M. Solleveld, "Periodic cyclic homology of reductive $p$-adic groups", J. Noncommutative Geometry 3.4 (2009), 501-558.

[Sol2] M. Solleveld, "Parabolically induced representations of graded Hecke algebras", Algebras and Representation Theory 15.2 (2012), 233-271.

[Sol3] M. Solleveld, "On the classification of irreducible representations of affine Hecke algebras with unequal parameters", Representation Theory 16 (2012), 1-87.

[Sol4] M. Solleveld, "Hochschild homology of affine Hecke algebras", J. Algebra 384 (2013), 1-35.

[Sol5] M. Solleveld, "Topological K-theory of affine Hecke algebras", arXiv:1610.06722 (2016), to appear in Annals of K-theory.

[Vig] M.-F. Vignéras, "On formal dimensions for reductive p-adic groups", pp. 225-266 in: Festschrift in honor of I.I. Piatetski-Shapiro on the occasion of his sixtieth birthday, Part I, Israel Math. Conf. Proc. 2, Weizmann, Jerusalem, 1990.

[Wal] J.-L. Waldspurger, "La formule de Plancherel pour les groupes p-adiques (d'après HarishChandra)", J. Inst. Math. Jussieu 2.2 (2003), 235-333. 